Prepared for the U.S. Department of Energy

Under Contract DE-AC05-76RL01830

\title{
Plutonium Mobility Studies: 216-Z-9 Trench Sample Analysis Results
}

KJ Cantrell

KN Geiszler

BW Arey

September 2008

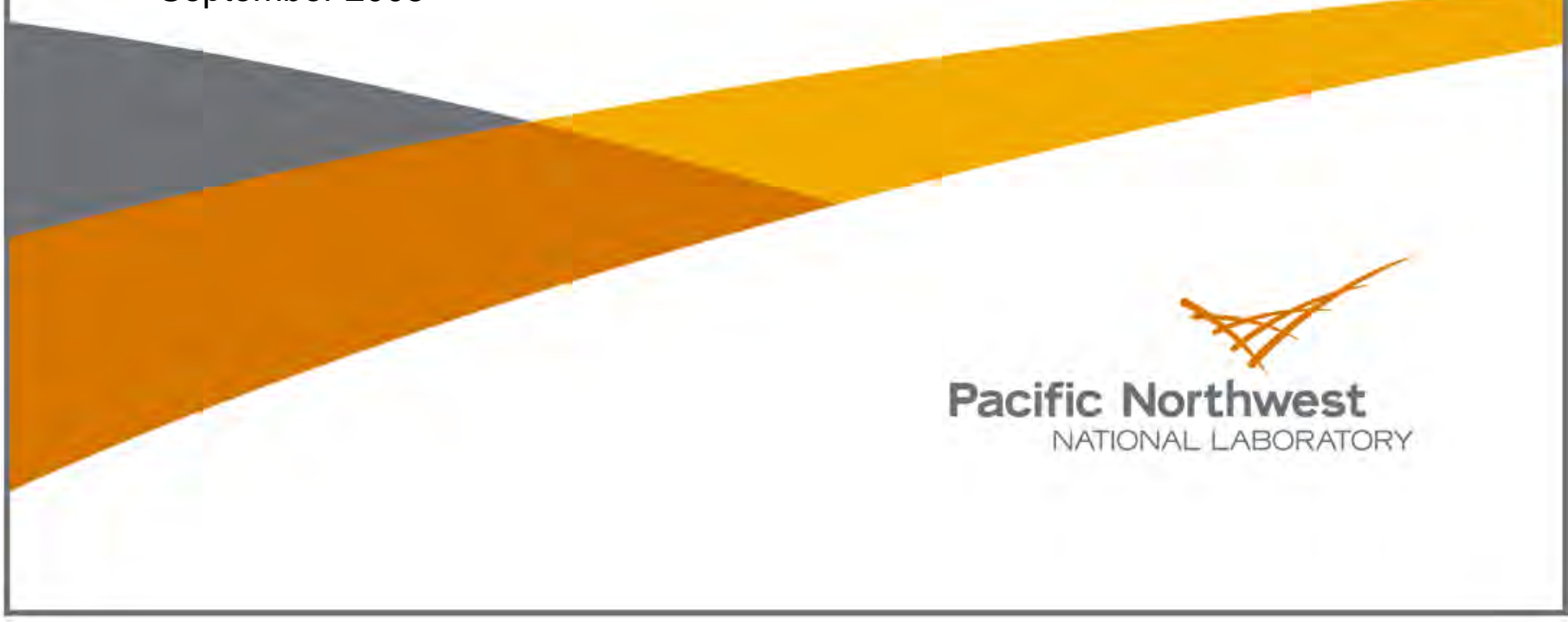




\section{Plutonium Mobility Studies: 216-Z-9 Trench Sample Analysis Results}

KJ Cantrell

KN Geiszler

BW Arey

September 2008

Prepared for Fluor Hanford, Inc. and the U.S. Department of Energy under Contract DE-AC05-76RL01830

Pacific Northwest National Laboratory Richland, Washington 99352 



\section{Executive Summary}

Fluor Hanford Inc requested the services of PNNL to provide technical support for remediation decisions concerning the 200-PW-1 Operable Unit group. Remedial investigations indicate that plutonium and americium have migrated through the vadose zone to the Cold Creek unit at a depth of approximately $110 \mathrm{ft}$ below ground surface (bgs). Because plutonium is commonly perceived to not be very mobile in the subsurface, it is important to demonstrate a fundamental understanding of how plutonium and americium moved to these depths in the vadose zone, and to determine under what conditions plutonium and americium could migrate further within the vadose zone, potentially to groundwater at a depth of approximately $225 \mathrm{ft}$ bgs at this location.

To address this issue Fluor Hanford, Inc. has requested Pacific Northwest National Laboratory (PNNL) conduct technical studies to demonstrate a thorough understanding of the geochemical behavior of plutonium and americium in the vadose zone at these sites. The waste sites in this operable unit group are unique in that they received wastes from various solvent extraction processes at the Z Plant Complex. These plutonium and americium contaminated wastes included acidic high salt wastes, organic rich wastes, and other more dilute aqueous wastes. The dominant components of the acidic high salt wastes were nitric acid, fluoride, aluminum, magnesium, and calcium. These high salt wastes were neutralized to $\mathrm{pH} 2.5$ with $\mathrm{NaOH}$ before disposal. Some of the organic components in the organic rich wastes included carbon tetrachloride, tributyl phosphate (TBP), various TBP degradation products, dibutyl butyl phosphonate, and lard oil.

This report presents results of laboratory characterization, testing, and analysis of selected sediment samples collected from two wells (299-W15-46 and 299-W15-48) drilled near the 216-Z-9 Trench to elucidate the form and potential for plutonium and americium to be mobilized under present conditions and in future remediation scenarios. Analyses included moisture content, determination of the less than sand-size fraction (silt plus clay), carbon analysis, scanning electron microscopy/energy dispersive spectrometry (SEM/EDS) analysis, microwave-assisted acid digestions for total element analysis, and sediment leaching tests with groundwater. Results of the leaching tests were used as input to conduct equilibrium geochemical modeling of the solutions with Geochemist's Workbench ${ }^{\circledR}$. Geochemical modeling results for plutonium were evaluated in terms of recent conclusions regarding the solubility and reduction-oxidation reactions of plutonium by Neck et al. (2007a, 2007b). ${ }^{1,2}$

The highest concentrations of plutonium and americium were associated with sediments of low silt/clay content and located above silt/clay rich layers within the sediment profile. It was also found that plutonium and americium were relatively enriched in the silt/clay portion of these samples. SEM/EDS analysis indicated the plutonium and americium in these sediments does not occur as discrete micron-size particles.

Leach testing of these sediment samples with Hanford groundwater indicates that release of plutonium and americium from the sediments is correlated most significantly with the final acidity of the

\footnotetext{
${ }^{1}$ Neck V, M Altmaier, A Seibert, JI Yun, CM Marquardt and T Fanghänel. 2007a. "Solubility and Redox Reactions of $\mathrm{Pu}(\mathrm{IV})$ Hydrous Oxides: Evidence for the Formation of $\mathrm{PuO}_{2+\mathrm{x}}(\mathrm{s}, \mathrm{hyd})$." Radiochimica Acta 95:193-207.

${ }^{2}$ Neck V, M Altmaier, and T Fanghänel. 2007b. "Solubility of Plutonium Hydroxides/Hydrous Oxides Under Reducing Conditions and in the Presence of Oxygen.” Comptes Rendus Chimie 10:959-977.
} 
extracts and not the initial concentrations of plutonium and americium in the sediments. Only extracts that were acidic after contact with the sediments ( $\mathrm{pH} 4.3$ to 5.4) contained detectable concentrations of leachable plutonium and americium. Groundwater leachates from the 216-Z-9 Trench sediment samples containing high concentrations of TBP suggest that if the TBP degradation products dibutyl phosphate and monobutyl phosphate are available in these sediments, they do not significantly increase the groundwater leachability of plutonium and americium.

Geochemical modeling results indicate the americium concentrations in water in contact with these sediments is highly undersaturated with respect to $\mathrm{Am}(\mathrm{OH})_{3}(\mathrm{c})$. It is likely that desorption of americium adsorbed to the sediments during the period of active waste water disposal is what controls americium concentrations in solutions in contact with these sediments. Sediment extracts that had measureable concentrations of americium only occurred in samples that were fairly acidic (pH 4.3 to 4.6), indicating that americium will remain effectively sequestered to sediments when $\mathrm{pH}$ conditions approach those of normal Hanford Site groundwater (mildly alkaline, pH 8).

The geochemical modeling results indicate that plutonium in extracts in contact with acidic sediment is significantly undersaturated with respect to $\mathrm{PuO}_{2}(\mathrm{am})$. However, recent reviews of plutonium solubility and reduction-oxidation reactions indicate data used for these calculations is incomplete (Neck et al. 2007a, 2007b). The results of Neck et al. (2007a, 2007b) suggest that plutonium concentrations in solutions in contact with the 216-Z-9 Trench sediment samples might be controlled by a mixed valent solid phase $\left[\left(\mathrm{Pu}^{\mathrm{V}}\right)_{2 \mathrm{x}}\left(\mathrm{Pu}^{\mathrm{IV}}\right)_{1-2 \mathrm{x}} \mathrm{O}_{2+\mathrm{x}}(\mathrm{am})\right]$ with various dissolved $\mathrm{Pu}(\mathrm{V})$ complexes and $\mathrm{Pu}(\mathrm{IV}) \mathrm{O}_{2}(\mathrm{am})$ colloids or nanoclusters being the dominant species in solution for typical Hanford Site groundwater conditions. Adsorption is likely to have a major impact on the potential for these species to remain in solution. Both $\mathrm{Pu}(\mathrm{V})$ complexes and $\mathrm{Pu}(\mathrm{IV}) \mathrm{O}_{2}(\mathrm{am})$ colloids or nanoclusters are well known for their high adsorption affinity for oxide and hydroxide mineral surfaces (Neck et al. 2007a, 2007b; Clark et al. 2006; Kaplan et al. 2006; Powell et al. 2005). ${ }^{3,4,5}$ As a result, these species are not likely to remain in solution as $\mathrm{pH}$ values approach those of typical Hanford Site groundwater (mildly alkaline, pH 8).

\footnotetext{
${ }^{3}$ Clark DL, SS Hecker, GD Jarvinen and MP Neu. 2006. "Plutonium.” Chapter 7 in The Chemistry of the Actinide and Transactinide Elements, $3^{\text {rd }}$ ed., pp. 813-1264, eds LR Morss, NM Edelstein, J Fuger, and JJ Katz, Springer, Netherlands.

${ }^{4}$ Kaplan DI, BA Powell, L Gumapas, JT Coates, RA Fjeld, and DP Diprete. 2006. "Influence of pH on Plutonium Desorption/Solubilization from Sediment.” Environmental Science \& Technology 40:5937-5942.

${ }^{5}$ Powell BA, RA Fjeld, DI Kaplan, JT Coates and SM Serkiz. 2005. " $\mathrm{Pu}(\mathrm{V}) \mathrm{O}_{2}^{+}$Adsorption and Reduction by Synthetic Hematite and Goethite.” Environmental Science \& Technology 39:2107-2114.
} 


\section{Acknowledgments}

The authors wish to acknowledge C. Sutton at Fluor Federal Services (Richland, Washington) and V. J. Rohay at Fluor Hanford, Inc. (Richland, Washington) for providing project funding and technical guidance. We greatly appreciate the technical reviews provided by T. M. Jones (Fluor Federal Services, Richland, Washington) and W. J. Deutsch (Pacific Northwest National Laboratory [PNNL]). The authors would also like to thank S. R. Baum, E. T. Clayton, C. Iovin, D. M. Smith and I. V. Kutnyakov (all of PNNL) for completing the chemical analyses of the samples from our studies. We are particularly grateful to H. E. Matthews (PNNL) for completing the editorial review and K. R. Neiderhiser (PNNL) for final word processing of this technical report. 



\section{Acronyms and Abbreviations}

$\begin{array}{ll}\text { am } & \text { amorphous } \\ \text { ASTM } & \text { American Society for Testing and Materials } \\ \text { DBP } & \text { below ground surface } \\ \text { DOE } & \text { dibutyl phosphate } \\ \text { EDS } & \text { U.S. Department of Energy } \\ \text { EPA } & \text { energy dispersive spectrometry } \\ \text { FY } & \text { U.S. Environmental Protection Agency } \\ \text { IC } & \text { fiscal year } \\ \text { ICP-MS } & \text { ion chromatography (chromatograph) } \\ \text { ICP-OES } & \text { inductively coupled plasma-mass spectrometry (spectrometer) } \\ \text { ID } & \text { inductively coupled plasma-optical emission spectroscopy (same as ICP-AES) } \\ \text { MBP } & \text { identification } \\ \text { pH } & \text { monobutyl phosphate } \\ \text { PNNL } & \text { measure of the acidity of a solution, where pH is the negative of the logarithm of } \\ \text { redox } & \text { the activity of H in solution } \\ \text { SEM } & \text { Pacific Northwest National Laboratory } \\ \text { SI } & \text { reduction-oxidation (process) } \\ \text { TBP } & \text { scanning electron microscopy (or microscope) } \\ \text { wt } & \text { saturation index } \\ & \text { tributyl phosphate } \\ \text { weight }\end{array}$





\section{Units of Measure}

$\begin{array}{ll}\text { Eh } & \text { reduction oxidation potential } \\ \mathrm{ft} & \text { foot } \\ \mathrm{g} & \text { gram } \\ \mathrm{keV} & \text { kiloelectron volt } \\ \mathrm{L} & \text { liter } \\ \mu & \text { micro (prefix, 10-6) } \\ \mu \mathrm{Ci} & \text { microCurie } \\ \mu \mathrm{g} & \text { microgram } \\ \mu \mathrm{m} & \text { micrometer } \\ \mathrm{M} & \text { molarity, mol/L } \\ \mathrm{mg} & \text { milligram } \\ \mathrm{mL} & \text { milliliter } \\ \mathrm{mM} & \text { molarity, millimol/L } \\ \mathrm{wt} \% & \text { weight percent }\end{array}$





\section{Contents}

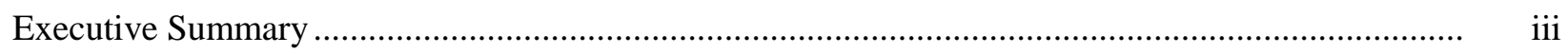

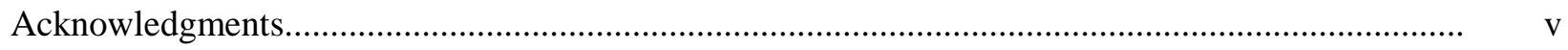

Acronyms and Abbreviations .................................................................................................... vii

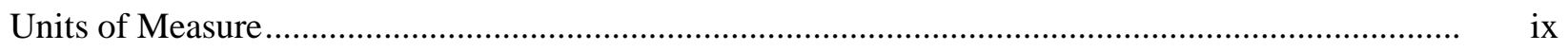

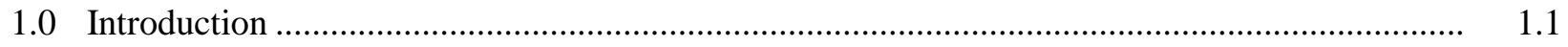

2.0 Materials and Laboratory Test Methods ............................................................................. 2.1

2.1 216-Z-9 Trench Sediment Samples .............................................................................. 2.1

2.2 Moisture Content ................................................................................................... 2.1

2.3 Sediment Size Fractionation - Less Than Sand-Size Content ........................................ 2.2

2.4 Carbon Analysis................................................................................................ 2.2

2.5 Scanning Electron Microscopy/Energy Dispersive Spectroscopy Analysis....................... 2.3

2.6 Microwave-Assisted Acid Digestion ............................................................................ 2.4

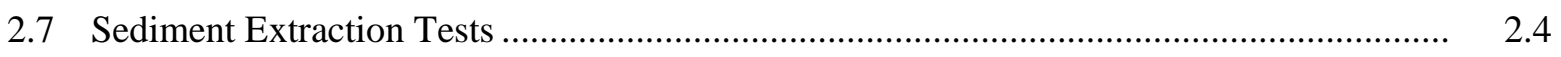

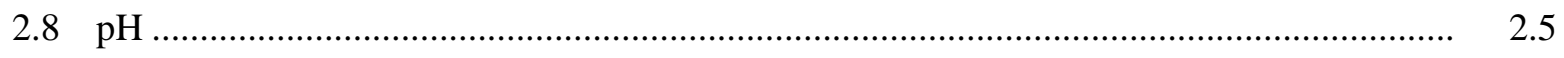

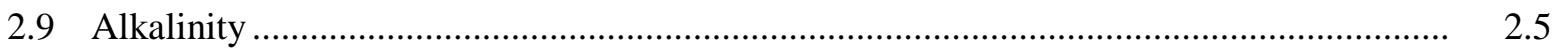

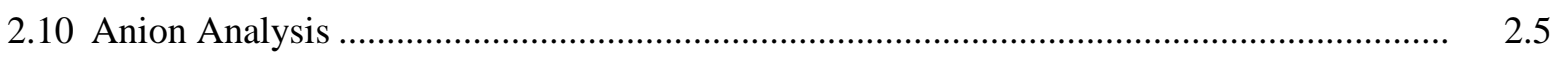

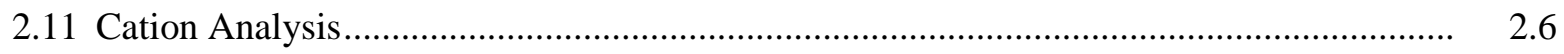

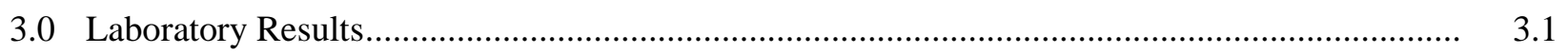

3.1 Whole Sediment Results ...................................................................................

3.1.1 Moisture Content .............................................................................................. 3.1

3.1.2 Less than Sand-Size Fraction Determination Results ........................................... 3.1

3.1.3 Carbon Analysis Results .............................................................................. 3.2

3.1.4 Scanning Electron Microscopy/Energy Dispersive Spectrometry Results............... 3.3

3.2 Acid Digestion Results ............................................................................................

3.2.1 Elemental Analysis of Acid Digestions.............................................................. 3.3

3.2.2 Plutonium and Americium in Acid Digestions .................................................... 3.4

3.3 Sediment Extract Results ......................................................................................... 3.5

3.3.1 Alkalinity and $\mathrm{pH}$ in Sediment Extracts ........................................................ 3.5

3.3.2 Anions in Sediment Extracts ............................................................................. 3.6

3.3.3 Elemental Analysis of Sediment Extracts ........................................................ 3.7

3.3.4 Plutonium and Americium in Sediment Extracts ................................................. 3.9

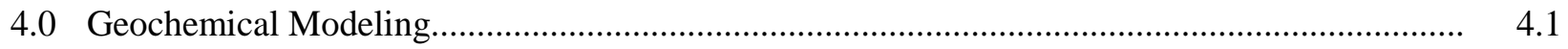

5.0 Summary and Conclusions ............................................................................................

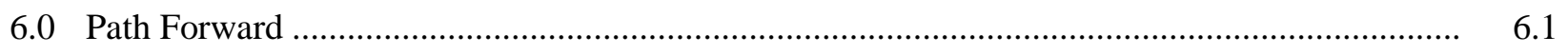

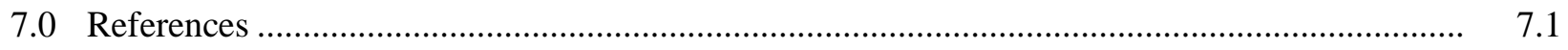

Appendix A - Geochemical Modeling Output Files.................................................................... A.1 


\section{Figures}

1.1 Location of Wells in the Vicinity of the 216-Z-9 Trench

\section{Tables}

2.1 Samples Selected for Analysis, Well ID .......................................................................... 2.1

2.2 Initial Composition of Groundwater Used in Extraction Tests ................................................... 2.5

3.1 Moisture Content of Sediment Samples................................................................................

3.2 Less Than Sand-Size Content of Sediment Samples............................................................. 3.1

3.3 Total Inorganic and Total Organic Carbon in Sediment Samples............................................ 3.2

3.4 Major Element Concentrations in Sediments Determined by ICP-OES Analysis of

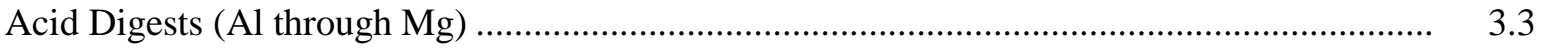

3.5 Major Ion Concentrations in Sediments Determined by ICP-OES Analysis of Acid

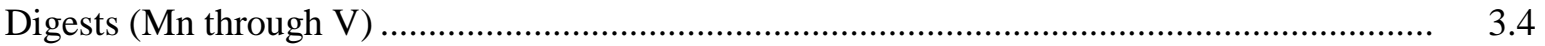

3.6 Pu and Am Concentrations in Sediments Determined by ICP-MS Analysis of Acid

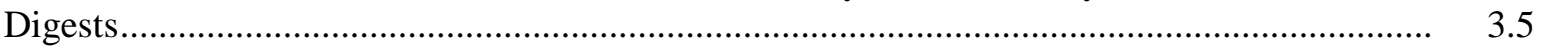

$3.7 \mathrm{pH}$ and Alkalinity of Sediment Extracts …........................................................................ 3.6

3.8 Major Anion Concentrations in Sediment Extracts Determined by IC and Extracted Concentration in the Sediments ....................................................................................... 3.7

3.9 Element Concentrations in Sediment Extracts Determined by ICP-OES (Al through Mg) .......... 3.8

3.10 Element Concentrations in Sediment Extracts Determined by ICP-OES (Mn through Sr)........... 3.8

3.11 Pu and Am Concentrations in Sediment Extracts Determined by ICP-MS and Extracted Concentration in the Sediments ........................................................................................ 3.10

4.1 Results of Thermodynamic Equilibrium Modeling............................................................ 


\subsection{Introduction}

Fluor Hanford, Inc. requested the services of Pacific Northwest National Laboratory (PNNL) to provide technical support for remediation decisions concerning the 200-PW-1 Operable Unit group. Remedial investigations indicate that plutonium and americium have migrated through the vadose zone to the Cold Creek unit at a depth of approximately $110 \mathrm{ft}$ below ground surface (bgs). Because plutonium is commonly perceived to not be very mobile in the subsurface, it is important to demonstrate a fundamental understanding of how plutonium and americium moved to these depths in the vadose zone, and to determine under what conditions plutonium and americium could migrate further within the vadose zone, potentially to groundwater at a depth of approximately $225 \mathrm{ft}$ bgs at this location.

To address this issue Fluor Hanford, Inc. has requested PNNL conduct technical studies to demonstrate a thorough understanding of the geochemical behavior of plutonium and americium in the vadose zone at these sites. The waste sites in this operable unit group are unique in that they received wastes from various solvent extraction processes at the Z Plant Complex. These plutonium and americium contaminated wastes included acidic high salt wastes, organic rich wastes, and other more dilute aqueous wastes. The dominant components of the acidic high salt wastes were nitric acid, fluoride, aluminum, magnesium, and calcium. These high salt wastes were neutralized to $\mathrm{pH} 2.5$ with $\mathrm{NaOH}$ before disposal. Some of the organic components in the organic rich wastes included carbon tetrachloride, tributyl phosphate (TBP), various TBP degradation products, dibutyl butyl phosphonate, and lard oil.

This report presents results of testing and analysis conducted in fiscal year (FY) 2008 on sediment samples collected from two wells: 299-W15-46 (borehole C3426) and 299-W15-48 (borehole C3427) drilled at the 216-Z-9 Trench. This work was conducted to further evaluate the form and potential for plutonium and americium to be mobilized under present conditions and those contaminants that could be expected in future remediation scenarios. Wells 299-W15-46 and 299-W15-48 were drilled to collect soil and soil-vapor samples to better define the stratigraphy, and to characterize the nature and extent of contamination at the 216-Z-9 Trench (Caron 2005, Bowles and Rohay 2007). Locations of these wells are indicated in Figure 1.1. Well 299-W15-46 is located approximately $30 \mathrm{ft}$ south of the trench. Well 299-W15-48, which was drilled as a slant borehole, starts $18 \mathrm{ft}$ east of the trench and extends below the trench westerly at an angle of 32 degrees from vertical. Drilling of well 299-W15-46 started in October 2003 and was completed in May 2005. Drilling of well 299-W15-48 began in February 2006 and was completed in May 2006. Additional background information on these wells is provided in Caron (2005) and Bowles and Rohay (2007). 


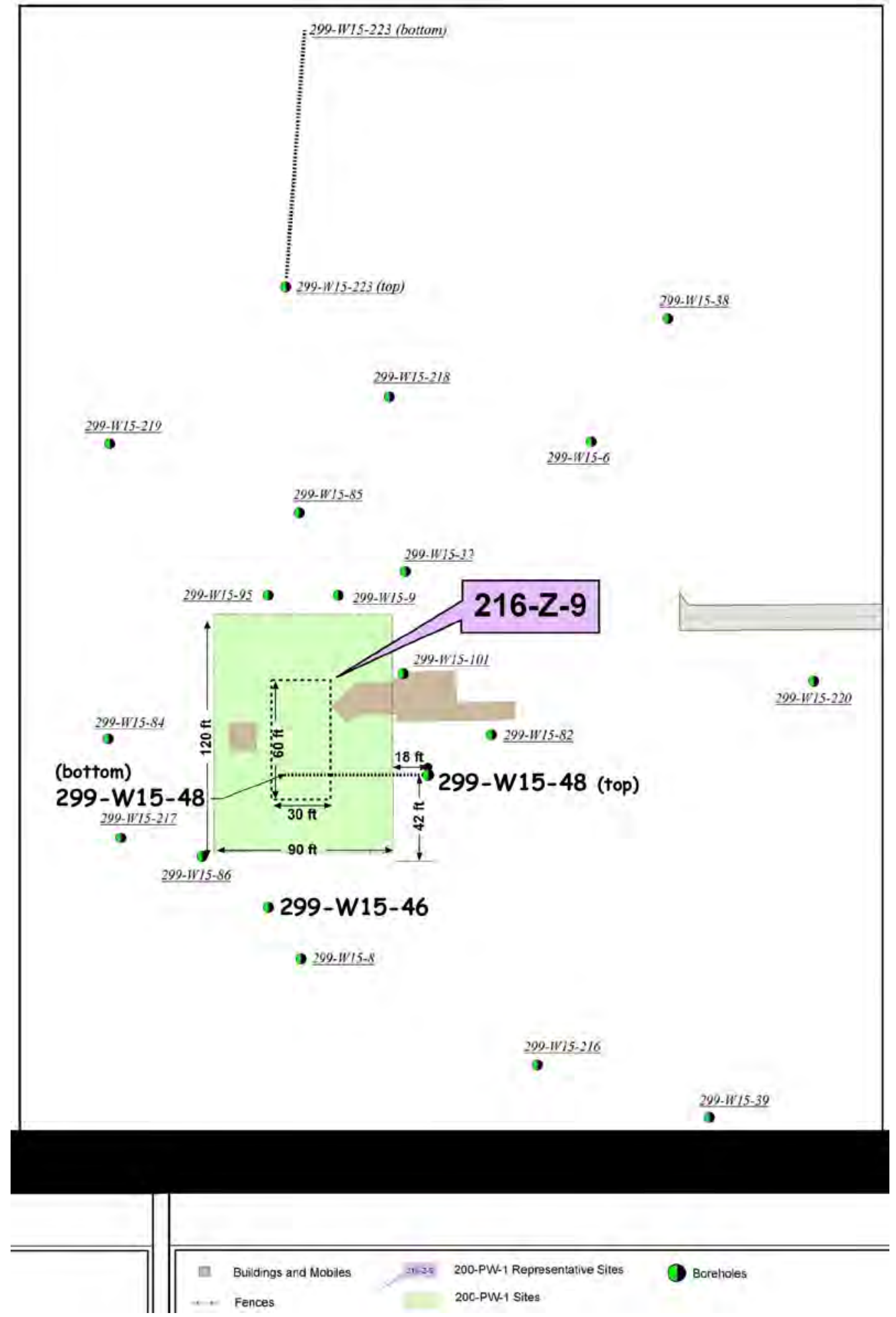

Figure 1.1. Location of Wells in the Vicinity of the 216-Z-9 Trench 


\subsection{Materials and Laboratory Test Methods}

\section{$2.1 \quad 216-Z-9$ Trench Sediment Samples}

The 216-Z-9 Trench sediment samples selected for analysis in this study are listed in Table 2.1. Included are well identification (ID) numbers, downhole depth, and depth below ground surface (bgs). Well 299-W15-46 was drilled vertically, so the downhole depth and the bgs depth are the same, whereas well 299-W15-48 was drilled at an angle of 32 degrees from vertical, resulting in downhole depths that are greater than bgs depths. In some cases, subsamples of these samples were prepared by separating the less than sand-size fraction (silt plus clay fraction) from the bulk sample. These samples were prepared by collecting the material that passed through a $53-\mu \mathrm{m}$ sieve and are indicated as fine in the sample ID. These samples were stored for several years in sealed cores (unrefrigerated). It does not appear that this has had any significant impact on the results reported here.

Table 2.1. Samples Selected for Analysis, Well ID

\begin{tabular}{cccc}
\hline Sample ID & Well ID & $\begin{array}{c}\text { Depth (ft) } \\
\text { (Downhole) }\end{array}$ & $\begin{array}{c}\text { Depth (ft) } \\
\text { (Below Ground } \\
\text { Surface) }\end{array}$ \\
\hline B17RF2 C4 & 299-W15-46 & $50.5-51.0$ & $50.5-51.0$ \\
B17RF2 C1 & 299-W15-46 & $52.0-52.5$ & $52.0-52.5$ \\
B17N54 & 299-W15-46 & $107.0-109.0$ & $107.0-109.0$ \\
B17N59 & 299-W15-46 & $115.0-117.5$ & $115.0-117.5$ \\
B17N66 & 299-W15-46 & $122.0-124.5$ & $122.0-124.5$ \\
\hline B1HK27 & 299-W15-48 & $67.0-69.0$ & $56.8-58.5$ \\
B1HK32 & 299-W15-48 & $70.0-72.0$ & $59.4-61.1$ \\
B1HK52 & 299-W15-48 & $100.0-102.0$ & $84.8-86.5$ \\
B1HK42 & 299-W15-48 & $118.5-120.5$ & $100.5-102.2$ \\
B1HK57 & 299-W15-48 & $122.5-124.5$ & $103.9-105.6$ \\
\hline
\end{tabular}

\subsection{Moisture Content}

The moisture contents of the sediment samples were measured to calculate dry weight concentrations for constituents in the sediments. Dry weight concentrations provide a consistent measurement unit for comparison purposes that eliminates the effect of variable water content on sample concentrations.

Gravimetric water content of the sediment was determined using American Society for Testing and Materials (ASTM) procedure, Standard Test Method for Laboratory Determination of Water (Moisture) Content of Soil and Rock by Mass (ASTM D2216-98, 1998) with the following minor exceptions: 1) the volume of sample recommended was decreased due to radiological concerns; and 2) the sample was dried at a lower oven temperature, $105^{\circ} \mathrm{C}$, for a longer period of time to prevent dehydration of the solids. 
Sediment samples were placed in tared containers, weighed, and dried in an oven until a constant weight was achieved, usually 24 to 48 hours. The container was then removed from the oven, sealed, cooled, and weighed. All measurements were performed using a calibrated balance. The gravimetric water content is computed as the percentage change in sediment sample weight before and after oven drying (i.e., [ [wet weight - dry weight $\} /$ dry weight]).

\subsection{Sediment Size Fractionation - Less Than Sand-Size Content}

The less than sand-size (silt plus clay fraction) was determined by weighing an aliquot of the dry bulk sample, sieving this sample with a 53- $\mu \mathrm{m}$ sieve, and then weighing the sample quantity that passed through the sieve. The percent less than sand-size fraction was calculated by dividing the mass of the sample that passed through the sieve by the mass of the total sample and multiplying by 100 .

\subsection{Carbon Analysis}

The total carbon concentration in aliquots of sediment was measured with a Shimadzu TOC-V CSN instrument with a SSM-5000A Total Organic Carbon Analyzer by combustion at approximately $900^{\circ} \mathrm{C}$ based on the ASTM procedure, Standard Test Methods for Analysis of Metal Bearing Ores and Related Materials by Combustion Infrared Absorption Spectrometry (ASTM E1915-01, 2001). ${ }^{1}$ Samples were placed into pre-combusted, tared, ceramic combustion sample holders and weighed on a calibrated balance. After the combustion sample holders were placed into the furnace introduction tube, an approximately 2-minute waiting period was allowed for the ultra-pure oxygen carrier gas to remove any carbon dioxide introduced to the system from the atmosphere during sample placement. After this sparging process, the sample was moved into the combustion furnace and the combustion was started. The carrier gas then delivered the sample combustion products to the cell of a non-dispersive infrared gas analyzer where the carbon dioxide was detected and measured. The amount of carbon dioxide measured is proportional to the total carbon content of the sample. Adequate system performance was confirmed by analyzing known quantities of a calcium carbonate standard.

Sediment samples were analyzed for inorganic carbon content by placing an aliquot of sediment into a ceramic combustion boat. The combustion boat was placed into the sample introduction tube where it was sparged with ultra-pure oxygen for 2 minutes to remove atmospheric carbon dioxide. A small amount (usually $0.6 \mathrm{ml}$ ) of $3 \mathrm{M}$ phosphoric acid was then added to the sample in the combustion boat. The boat was moved into the combustion furnace where it was heated to $200^{\circ} \mathrm{C}$. Samples were completely covered by the acid to allow full reaction to occur. Ultra-pure oxygen swept the resulting carbon dioxide through a dehumidifier and scrubber into the cell of a non-dispersive infrared gas analyzer where the carbon dioxide was detected and measured. The amount of $\mathrm{CO}_{2}$ measured is proportional to the inorganic carbon content of the sample. Organic carbon content was determined by the difference between the inorganic carbon and total carbon concentrations.

\footnotetext{
${ }^{1}$ Unpublished technical procedure: Operating of Carbon Analyzer (TOC-V + SSM-5000A + ASI (Shimadzu)). AGG-TOC-001, Pacific Northwest National Laboratory, Richland, Washington.
} 


\subsection{Scanning Electron Microscopy/Energy Dispersive Spectroscopy Analysis}

Scanning electron microscopy/energy dispersive spectroscopy (SEM/EDS) was used to obtain high-resolution images of the morphologies, sizes, surface textures, and composition of micrometer/ submicrometer-sized particles present in the sediment samples. The primary goal of the analyses was to determine elemental compositions of plutonium- and americium-containing particles, and any phase associations or elemental correlations of the plutonium and americium with other components in the sediments. The information could help to determine the form of plutonium and americium in the contaminated sediments and any possible associations with organo-phosphate compounds.

SEM mounts were prepared for four samples (B1HK32 Bulk, B1HK32 Fine, B1HK42 Bulk, and B1HK42 Fine). These samples were selected because B1HK32 had the highest plutonium concentration and B1HK42 had the highest americium concentration of any of the samples previously analyzed (DOE/RL 2007). The mounts used for SEM/EDS consisted of double-sided carbon tape attached to standard aluminum-mounting stubs. For each mount, small aliquots of each sediment sample were placed on the exposed upper surface of the carbon tape using a micro spatula. Each mount was then coated with carbon using a vacuum sputter-coater to improve the conductivity of the samples and thus, the quality of the SEM images and EDS signals.

A JEOL JSM-840 SEM was used for high-resolution imaging of micrometer/submicrometer-sized particles in the sediment samples. The EDS system provided qualitative elemental analysis for scanned areas of particles. The SEM is equipped with an INCA Energy EDS System to automate the collection of EDS spectra over multi-micrometer-sized areas of an SEM-imaged sample. ${ }^{2}$ The EDS software was calibrated to a copper reference standard mounted on a specimen holder. Operating conditions consisted of $20 \mathrm{keV}$ for SEM imaging and $20 \mathrm{keV}, 100$ live seconds $^{3}$ for the EDS analyses. The EDS analyses are limited to elements with atomic weights heavier than boron (B). Compositions determined by EDS are qualitative and have large uncertainties resulting from alignment artifacts caused by the variable sample and detector configurations that exist when different particles are imaged by SEM.

Photomicrographs of high-resolution backscattered electron images were obtained as digital images and stored in electronic format. To help identify particles that contain elements with large atomic numbers, such as plutonium and americium, the SEM was operated in the backscattered electron mode. Backscattered electron emission intensity is a function of the element's atomic number-the larger the atomic number, the brighter the signal.

Each SEM mount was examined by the SEM to identify those particles and surface features that were typical or unusual for the sample. During this examination, a SEM micrograph was first recorded at low magnification for a representative area of the mount to provide a general perspective of the sizes, types, and distributions of particles that comprise each SEM mount. Additional SEM micrographs were then

\footnotetext{
${ }^{2}$ INCA Energy EDS System is a product of Oxford Instruments, Concord, Massachusetts.

${ }^{3}$ Live time is when (real time less dead time) the EDS system is available to detect incoming x-ray photons. Dead time is the portion of the total analyzing time that is actually spent processing or measuring $x$-rays. While each $\mathrm{x}$-ray pulse is being measured, the system cannot measure another x-ray that may enter the detector and is therefore referred to as "dead."
} 
recorded of particles at greater magnifications to provide a more detailed representation of the particles' characteristics. Selected points on these particles were then analyzed by EDS.

\subsection{Microwave-Assisted Acid Digestion}

Bulk compositions of sediment solids were determined using a U.S. Environmental Protection Agency (EPA) microwave-assisted acid-digestion method-EPA SW-846 Method 3052 (EPA 1996) with substitutions. The substitutions to EPA SW-846 Method 3052 consist of 1) decreasing the concentrated nitric acid $\left(\mathrm{HNO}_{3}\right)$ volume from $9 \mathrm{~mL}$ to $5 \mathrm{~mL}$, and 2) increasing the double-deionized water volume from the recommended volume of 0 to $5 \mathrm{~mL}$ up to $10 \mathrm{~mL}$. This method was used to measure metals concentrations and a limited number of nonmetals (e.g., P and S) in the sediment. This method is not appropriate for the anion concentrations (e.g., $\mathrm{NO}_{3}, \mathrm{Cl}, \mathrm{F}$, and $\mathrm{BO}_{3}$ ) because of the addition of acids used in the procedure and analyses.

The basic acid-digestion procedure is described in EPA SW-846 Method 3052 (EPA 1996). In this procedure, $300 \mathrm{mg}$ of the sample is placed in a Teflon ${ }^{\circledR}$ microwave digestion vessel; $10-\mathrm{mL}$ water, 5 -mL $16 \mathrm{M} \mathrm{HNO}_{3}, 2-\mathrm{mL} 12 \mathrm{M} \mathrm{HCl}$, and 1-mL $29 \mathrm{M} \mathrm{HF}$ are added to the sample. ${ }^{4}$ The vessel is sealed and placed in a microwave-assisted digestion system. The samples are treated at the EPA-recommended temperatures and times. The sample is then allowed to cool, and $0.45 \mathrm{~g}$ of boric acid $\left(\mathrm{H}_{3} \mathrm{BO}_{3}\right)$ is added to the digestate and shaken by hand. Samples are filtered through a $0.45-\mu \mathrm{m}$ pore-size syringe filter prior to ICP-OES and ICP-MS analysis. There were no visible solids when the digestions were complete.

\subsection{Sediment Extraction Tests}

Water-soluble inorganic constituents were determined using a sediment extraction method in which Hanford Site groundwater was used as the leaching solution. In this method, $30 \mathrm{~mL}$ of Hanford Site groundwater (from monitoring well 699-49-100C) was added to a quantity of sediment ranging from 24 to $32 \mathrm{~g}$ contained in a 125-mL nalgene bottle. The nalgene bottle was sealed, briefly shaken by hand, and then placed on a mechanical orbital shaker for 1 day or 30 days. After shaking for the predetermined time, the bottle was placed in a centrifuge and spun at 4,000 revolutions per minute for 20 minutes. The supernatant was carefully decanted and filtered through a $0.45-\mu \mathrm{m}$ pore-size membrane. More details are provided in ASTM procedure, Standard Test Method for Shake Extraction of Solid Waste with Water (ASTM D3987-85, 1999). The initial major ion composition of the well 699-49-100C groundwater is shown in Table 2.2.

To evaluate the potential that colloidal size particulates could form and act as a transport vehicle for plutonium and americium in groundwater, three sediment samples were selected (B17RF2 C1, B1HK32, and B1HK42) for extraction using the procedure described above. The leachates were first filtered through a $0.45-\mu \mathrm{m}$ pore-size membrane. Aliquots of these filtered samples were taken for analysis. Other aliquots of the filtered samples were then filtered with Amicon Centriplus cones (Amicon Corp.) with a 25,000-molecular-weight cutoff (approximately 0.0018 - $\mu$ m pore size), which were then analyzed. Prior to use, the filters were washed with deionized water. Only ${ }^{239} \mathrm{Pu}$ and ${ }^{241} \mathrm{Am}$ were analyzed in these solutions.

\footnotetext{
${ }^{4}$ Teflon is a registered trademark of the E.I. du Pont de Nemours and Company.
} 
Table 2.2. Initial Composition of Groundwater (from well 699-49-100C) Used in Extraction Tests

\begin{tabular}{cc}
\hline Constituent & Concentration $(\mathrm{mg} / \mathrm{L})$ \\
\hline $\mathrm{pH}$ & 8.04 \\
Alkalinity (as $\left.\mathrm{CaCO}_{3}\right)$ & 187 \\
$\mathrm{Cl}$ & 18.9 \\
$\mathrm{NO}_{3}$ & 9.44 \\
$\mathrm{SO}_{4}$ & 70.6 \\
$\mathrm{~F}$ & 0.45 \\
$\mathrm{Na}$ & 24.4 \\
$\mathrm{~K}$ & 7.50 \\
$\mathrm{Ca}$ & 59.5 \\
$\mathrm{Mg}$ & 22.0 \\
$\mathrm{Sr}$ & 0.24 \\
$\mathrm{Si}$ & 28.0 \\
\hline
\end{tabular}

\section{$2.8 \mathrm{pH}$}

The $\mathrm{pH}$ of the solutions was measured using EPA SW-846, Method 9040C (EPA 2004) with a modification. The modification consists of using a solid-state $\mathrm{pH}$ electrode instead of the recommended glass electrode.

\subsection{Alkalinity}

Sample alkalinity was measured by standard titration. A volume of standardized sulfuric acid was added to the sample to an endpoint of $\mathrm{pH} 4.5$ to measure total alkalinity. Alkalinity is reported in terms of an equivalent mass of $\mathrm{CaCO}_{3}$. The alkalinity procedure follows Standard Method $2320 \mathrm{~B}$, “Alkalinity by Titration” (Clesceri et al. 1998).

\subsection{Anion Analysis}

Anion analysis was performed using an ion chromatograph (IC) following the technical procedure AGG-IC-001 (Lindberg 2004). ${ }^{5}$ Chloride, $\mathrm{NO}_{2}, \mathrm{Br}, \mathrm{NO}_{3}, \mathrm{SO}_{4}$, and $\mathrm{PO}_{4}$ were separated on a Dionex AS17 column with a gradient elution technique from 1-mM to 35-mM NaOH and measured using a conductivity detector. This methodology is a substitution for EPA SW-846, Method 9056A (EPA 2007) with the exception of using gradient elution with $\mathrm{NaOH}$ instead of the recommended isocratic elution with a $\mathrm{HCO}_{3}$ buffer.

\footnotetext{
${ }^{5}$ Lindberg MJ. 2004. Determinations by Ion Chromatography (IC). AGG-IC-001 (Rev. 0). Unpublished PNNL technical procedure, Pacific Northwest National Laboratory, Richland, Washington.
} 


\subsection{Cation Analysis}

Major cation analysis (including aluminum, silicon, calcium, magnesium, sodium, potassium, iron, and manganese) was performed by inductively coupled plasma-optical emission spectroscopy (ICP-OES) following PNNL-AGG-ICP-AES (Baum 2008). ${ }^{6}$ Selected radionuclide analysis $\left({ }^{239} \mathrm{Pu}\right.$ and ${ }^{241} \mathrm{Am}$ isotopes) was performed by ICP-mass spectrometry (MS) following PNNL-AGG-415 (Clayton 2008). ${ }^{7}$

For both ICP-OES and ICP-MS, high-purity calibration standards were used to generate calibration curves, and to verify continuing calibration during the analysis. Dilutions of 10 and 5 times were made for each sample and analyzed to investigate and correct for matrix interferences.

${ }^{6}$ Baum SR. 2008. Inductively Coupled Plasma -Optical Emission Spectrometry (ICP-OES) Analysis. PNNL-AGG-ICP-AES (Rev. 2). Unpublished PNNL technical procedure, Pacific Northwest National Laboratory, Richland, Washington.

${ }^{7}$ Clayton ET. 2008. "Inductively Coupled Plasma Mass Spectrophotometry (ICP-MS) Analysis.” PNNL-AGG-415 (Rev. 2). Unpublished PNNL technical procedure, Pacific Northwest National Laboratory, Richland, Washington. 


\subsection{Laboratory Results}

\subsection{Whole Sediment Results}

\subsubsection{Moisture Content}

Moisture content results, shown in Table 3.1, were used to calculate sediments concentrations on a dry weight basis. The moisture contents ranged from a low of 1.4\% (sample B1HK57) to a high of 14.5\% (sample B17N59). Because these samples were stored for a number of years, the moisture contents reported here may not be representative of the original moisture content of the samples collected in the field.

Table 3.1. Moisture Content of Sediment Samples

\begin{tabular}{cc}
\hline Sample ID & Percent Moisture \\
\hline B17RF2 C4 & 10.1 \\
B17RF2 C1 & 3.25 \\
B17N54 & 6.96 \\
B17N59 & 14.5 \\
B17N66 & 3.57 \\
\hline B1HK27 & 3.55 \\
B1HK32 & 1.35 \\
B1HK52 & 6.22 \\
B1HK42 & 6.81 \\
B1HK57 & 1.40 \\
\hline
\end{tabular}

\subsubsection{Less than Sand-Size Fraction Determination Results}

The less than sand-size fraction (silt plus clay fraction) determinations for the sediment samples are shown in Table 3.2. The range is from $0.00 \mathrm{wt} . \%$ in sample B17RF2 C1 to $39.3 \mathrm{wt} . \%$ in sample B1HK57. The highest percentages of less than sand-size fraction occur in samples collected at depths where the Cold Creek Unit is known to occur (B17N66, B1HK52, B1HK42, and B1HK57).

Table 3.2. Less Than Sand-Size Content of Sediment Samples (weight percent)

\begin{tabular}{ccc}
\hline Sample ID & Depth (bgs) & $\begin{array}{c}\text { Percent Silt and Clay } \\
\text { (By Weight) }\end{array}$ \\
\hline B17RF2 C4 & $50.5-51.0$ & 4.04 \\
B17RF2 C1 & $52.0-52.5$ & 0.00 \\
B17N54 & $107.0-109.0$ & 8.00 \\
B17N59 & $115.0-117.5$ & 6.04 \\
B17N66 & $122.0-124.5$ & 18.0 \\
\hline
\end{tabular}


Table 3.2. (contd)

\begin{tabular}{ccc}
\hline Sample ID & Depth (bgs) & $\begin{array}{c}\text { Percent Silt and Clay } \\
\text { (By Weight) }\end{array}$ \\
\hline B1HK27 & $56.8-58.5$ & 4.48 \\
B1HK32 & $59.4-61.1$ & 2.60 \\
B1HK52 & $84.8-86.5$ & 30.4 \\
B1HK42 & $100.5-102.2$ & 21.4 \\
B1HK57 & $103.9-105.6$ & 39.3 \\
\hline
\end{tabular}

\subsubsection{Carbon Analysis Results}

Total inorganic carbon and total organic carbon are presented in Table 3.3. Inorganic carbon results are generally below the detection limit, except for sample B17N59, B1HK42, and B1HK57, which occur in the lower Cold Creek Unit that contains a calcite rich layer. Total organic carbon results are generally near background concentrations, except for sample B1HK32. Previous analyses (DOE/RL 2007) indicate that exceptionally high tributyl phosphate (TBP) concentrations (3,000 $\mu \mathrm{g} / \mathrm{g}$ TBP) occur in this sample.

Table 3.3. Total Inorganic and Total Organic Carbon in Sediment Samples

\begin{tabular}{|c|c|c|}
\hline \multirow[b]{2}{*}{ Sample ID } & Total Inorganic Carbon & Total Organic Carbon \\
\hline & \multicolumn{2}{|c|}{$\mu \mathrm{g} / \mathrm{g}$-dry wt } \\
\hline B17RF2 C4 & $<200$ & 802 \\
\hline B17RF2 C4 dup & $<200$ & 508 \\
\hline B17RF2 C1 & $<200$ & 706 \\
\hline B17RF2 C1 dup & $<200$ & 592 \\
\hline B17N54 & $<200$ & 608 \\
\hline B17N54 dup & $<200$ & 621 \\
\hline B17N59 & 12,400 & $<200$ \\
\hline B17N59 dup & 8,750 & 355 \\
\hline B17N66 & $<200$ & 578 \\
\hline B17N66 dup & $<200$ & 578 \\
\hline B1HK27 & $<200$ & 586 \\
\hline B1HK27 dup & $<200$ & 616 \\
\hline B1HK32 & $<200$ & 2,670 \\
\hline B1HK32 dup & $<200$ & 2,380 \\
\hline B1HK52 & $<200$ & 483 \\
\hline B1HK52 dup & $<200$ & 588 \\
\hline B1HK42 & 575 & 491 \\
\hline B1HK42 dup & 537 & 491 \\
\hline B1HK57 & 2,970 & $<200$ \\
\hline B1HK57 dup & 2,910 & $<200$ \\
\hline
\end{tabular}




\subsubsection{Scanning Electron Microscopy/Energy Dispersive Spectrometry Results}

Four samples were examined by SEM/EDS (B1HK32 Bulk, B1HK32 Fine, B1HK42 Bulk, and B1HK42 Fine). A total of 144 spot EDS analyses were conducted on these samples. In no case was plutonium or americium detectable. As a result, no phase or elemental associations could be established with this technique; however, these results indicate that plutonium and americium do not occur as discrete micron or larger size particles.

\subsection{Acid Digestion Results}

\subsubsection{Elemental Analysis of Acid Digestions}

Sediment concentrations of major elements determined by ICP-OES analysis of the acid digestions are shown in Tables 3.4 and 3.5. Aluminum, barium, calcium, cadmium, chromium, iron, potassium, magnesium are shown in Table 3.4, while manganese, sodium, phosphorus, silicon, strontium, titanium, and vanadium are shown in Table 3.5. Only elements that had a result above the detection limit are shown. All results for silver, arsenic, beryllium, bismuth, cobalt, copper, lithium, molybdenum, nickel, lead, rhenium, sulfur, antimony, selenium, thallium, zinc, and zirconium were below the method quantification limit. For most elements, these results are not particularly remarkable and are typical of Hanford Site sediments. Phosphorus is noteworthy in that concentrations in the fine fraction of the sediments (less than sand-size material) are generally elevated relative to the bulk samples.

Table 3.4. Major Element Concentrations in Sediments Determined by ICP-OES Analysis of Acid Digests (Al through Mg)

\begin{tabular}{|c|c|c|c|c|c|c|c|c|}
\hline \multirow[b]{2}{*}{ Sample ID } & $\mathrm{Al}$ & $\mathrm{Ba}$ & $\mathrm{Ca}$ & Cd & $\mathrm{Cr}$ & $\mathrm{Fe}$ & K & $\mathrm{Mg}$ \\
\hline & \multicolumn{8}{|c|}{$\mu \mathrm{g} / \mathrm{g}$-dry sediment } \\
\hline B17RF2 C4 & $6.74 \mathrm{E}+04$ & $9.07 \mathrm{E}+02$ & $2.31 \mathrm{E}+04$ & $<5.24 \mathrm{E}+01$ & $<7.67 \mathrm{E}+01$ & $3.31 \mathrm{E}+04$ & $2.08 \mathrm{E}+04$ & $9.21 \mathrm{E}+03$ \\
\hline B17RF2 C4 Fine & $9.04 \mathrm{E}+04$ & $1.31 \mathrm{E}+03$ & $1.99 \mathrm{E}+04$ & $<1.12 \mathrm{E}+02$ & $<1.64 \mathrm{E}+02$ & $5.17 \mathrm{E}+04$ & $2.72 \mathrm{E}+04$ & $1.52 \mathrm{E}+04$ \\
\hline B17RF2 C1 & $6.67 \mathrm{E}+04$ & $8.33 \mathrm{E}+02$ & $1.58 \mathrm{E}+04$ & $<4.53 \mathrm{E}+01$ & $<6.63 \mathrm{E}+01$ & $2.59 \mathrm{E}+04$ & $2.21 \mathrm{E}+04$ & $7.33 \mathrm{E}+03$ \\
\hline B17N54 & $7.76 \mathrm{E}+04$ & $8.41 \mathrm{E}+02$ & $2.11 \mathrm{E}+04$ & $<5.05 \mathrm{E}+01$ & $<7.39 \mathrm{E}+01$ & $3.56 \mathrm{E}+04$ & $2.12 \mathrm{E}+04$ & $1.13 \mathrm{E}+04$ \\
\hline B17N54 Fine & $7.79 \mathrm{E}+04$ & $7.55 \mathrm{E}+02$ & $2.43 \mathrm{E}+04$ & $<6.27 \mathrm{E}+01$ & $<9.17 \mathrm{E}+01$ & $4.46 \mathrm{E}+04$ & $1.85 \mathrm{E}+04$ & $1.31 \mathrm{E}+04$ \\
\hline B17N59 & $6.64 \mathrm{E}+04$ & $5.18 \mathrm{E}+02$ & $6.04 \mathrm{E}+04$ & $2.43 \mathrm{E}+02$ & $<6.92 \mathrm{E}+01$ & $5.95 E+04$ & $1.35 \mathrm{E}+04$ & $1.53 \mathrm{E}+04$ \\
\hline B17N59 Fine & $7.36 \mathrm{E}+04$ & $5.98 \mathrm{E}+02$ & $6.27 \mathrm{E}+04$ & $2.60 \mathrm{E}+02$ & $<1.50 \mathrm{E}+02$ & $5.63 E+04$ & $1.74 \mathrm{E}+04$ & $1.62 \mathrm{E}+04$ \\
\hline B17N66 & $6.31 \mathrm{E}+04$ & $7.75 \mathrm{E}+02$ & $3.89 \mathrm{E}+04$ & $<3.77 \mathrm{E}+01$ & $<5.52 \mathrm{E}+01$ & $6.64 \mathrm{E}+04$ & $1.34 \mathrm{E}+04$ & $1.79 \mathrm{E}+04$ \\
\hline B17N66 Fine & $3.66 \mathrm{E}+04$ & $5.63 \mathrm{E}+02$ & $4.27 \mathrm{E}+04$ & $<4.69 \mathrm{E}+01$ & $<6.86 \mathrm{E}+01$ & $7.04 \mathrm{E}+04$ & $7.11 \mathrm{E}+03$ & $1.25 E+04$ \\
\hline B1HK27 & $5.92 E+04$ & $7.59 \mathrm{E}+02$ & $2.05 E+04$ & $<4.40 \mathrm{E}+01$ & $<6.44 \mathrm{E}+01$ & $3.94 \mathrm{E}+04$ & $1.88 \mathrm{E}+04$ & $9.19 \mathrm{E}+03$ \\
\hline B1HK27 Fine & $4.68 \mathrm{E}+04$ & $9.28 \mathrm{E}+02$ & $2.06 \mathrm{E}+04$ & $<5.21 \mathrm{E}+01$ & $9.13 \mathrm{E}+01$ & $4.42 \mathrm{E}+04$ & $1.48 \mathrm{E}+04$ & $9.03 E+03$ \\
\hline B1HK32 & $6.30 \mathrm{E}+04$ & $8.48 \mathrm{E}+02$ & $1.66 \mathrm{E}+04$ & $<4.34 \mathrm{E}+01$ & $<6.35 \mathrm{E}+01$ & $2.93 \mathrm{E}+04$ & $2.30 \mathrm{E}+04$ & $7.80 \mathrm{E}+03$ \\
\hline B1HK32 Fine & $6.25 E+04$ & $7.81 \mathrm{E}+02$ & $1.91 \mathrm{E}+04$ & $<4.31 \mathrm{E}+01$ & $<6.31 \mathrm{E}+01$ & $4.89 \mathrm{E}+04$ & $1.89 \mathrm{E}+04$ & $1.05 E+04$ \\
\hline B1HK52 & $6.13 \mathrm{E}+04$ & $8.13 \mathrm{E}+02$ & $1.69 \mathrm{E}+04$ & $<4.25 \mathrm{E}+01$ & $<6.23 \mathrm{E}+01$ & $3.43 \mathrm{E}+04$ & $2.04 \mathrm{E}+04$ & $7.49 \mathrm{E}+03$ \\
\hline B1HK52 Fine & $6.93 \mathrm{E}+04$ & $7.62 \mathrm{E}+02$ & $2.24 \mathrm{E}+04$ & $<4.12 \mathrm{E}+01$ & $<6.02 \mathrm{E}+01$ & $5.03 \mathrm{E}+04$ & $1.94 \mathrm{E}+04$ & $1.10 \mathrm{E}+04$ \\
\hline B1HK42 & $6.70 \mathrm{E}+04$ & $8.05 E+02$ & $2.01 \mathrm{E}+04$ & $<3.66 \mathrm{E}+01$ & $<5.36 \mathrm{E}+01$ & $3.37 \mathrm{E}+04$ & $1.93 \mathrm{E}+04$ & $9.82 E+03$ \\
\hline B1HK42 Fine & $7.22 \mathrm{E}+04$ & $8.06 \mathrm{E}+02$ & $2.85 \mathrm{E}+04$ & $<4.68 \mathrm{E}+01$ & $<6.85 \mathrm{E}+01$ & $4.70 \mathrm{E}+04$ & $1.76 \mathrm{E}+04$ & $1.22 \mathrm{E}+04$ \\
\hline B1HK57 & $6.95 \mathrm{E}+04$ & $7.84 \mathrm{E}+02$ & $2.32 \mathrm{E}+04$ & $<4.55 \mathrm{E}+01$ & $<6.65 \mathrm{E}+01$ & $4.01 \mathrm{E}+04$ & $2.17 \mathrm{E}+04$ & $1.16 \mathrm{E}+04$ \\
\hline B1HK57 Fine & $6.21 \mathrm{E}+04$ & $7.43 \mathrm{E}+02$ & $2.55 E+04$ & $<4.66 \mathrm{E}+01$ & $<6.83 \mathrm{E}+01$ & $4.29 \mathrm{E}+04$ & $2.03 E+04$ & $1.08 \mathrm{E}+04$ \\
\hline
\end{tabular}


Table 3.5. Major Ion Concentrations in Sediments Determined by ICP-OES Analysis of Acid Digests (Mn through V)

\begin{tabular}{cccccccr}
\hline & $\mathrm{Mn}$ & $\mathrm{Na}$ & $\mathrm{P}$ & $\mathrm{Si}$ & $\mathrm{Sr}$ & $\mathrm{Ti}$ & $\mathrm{V}$ \\
\cline { 2 - 8 } Sample ID & & \multicolumn{7}{c}{$\mu \mathrm{g} / \mathrm{g}$-dry sediment } \\
\hline B17RF2 C4 & $9.75 \mathrm{E}+02$ & $1.94 \mathrm{E}+04$ & $7.77 \mathrm{E}+02$ & $1.85 \mathrm{E}+05$ & $3.82 \mathrm{E}+02$ & $3.12 \mathrm{E}+03$ & $<8.54 \mathrm{E}+01$ \\
B17RF2 C4 Fine & $5.60 \mathrm{E}+02$ & $1.92 \mathrm{E}+04$ & $1.67 \mathrm{E}+03$ & $2.62 \mathrm{E}+05$ & $3.48 \mathrm{E}+02$ & $5.24 \mathrm{E}+03$ & $<1.82 \mathrm{E}+02$ \\
B17RF2 C1 & $3.52 \mathrm{E}+02$ & $2.06 \mathrm{E}+04$ & $5.61 \mathrm{E}+02$ & $1.53 \mathrm{E}+05$ & $3.98 \mathrm{E}+02$ & $2.56 \mathrm{E}+03$ & $<7.38 \mathrm{E}+01$ \\
B17N54 & $5.82 \mathrm{E}+02$ & $2.34 \mathrm{E}+04$ & $8.29 \mathrm{E}+02$ & $1.65 \mathrm{E}+05$ & $4.11 \mathrm{E}+02$ & $3.65 \mathrm{E}+03$ & $<8.23 \mathrm{E}+01$ \\
B17N54 Fine & $6.96 \mathrm{E}+02$ & $2.14 \mathrm{E}+04$ & $1.20 \mathrm{E}+03$ & $1.85 \mathrm{E}+05$ & $3.81 \mathrm{E}+02$ & $4.95 \mathrm{E}+03$ & $<1.02 \mathrm{E}+02$ \\
B17N59 & $9.35 \mathrm{E}+02$ & $1.91 \mathrm{E}+04$ & $5.12 \mathrm{E}+02$ & $1.54 \mathrm{E}+05$ & $3.66 \mathrm{E}+02$ & $8.71 \mathrm{E}+03$ & $1.28 \mathrm{E}+02$ \\
B17N59 Fine & $7.65 \mathrm{E}+02$ & $1.58 \mathrm{E}+04$ & $6.48 \mathrm{E}+02$ & $2.30 \mathrm{E}+05$ & $3.04 \mathrm{E}+02$ & $6.21 \mathrm{E}+03$ & $<1.67 \mathrm{E}+02$ \\
B17N66 & $1.06 \mathrm{E}+03$ & $2.18 \mathrm{E}+04$ & $1.11 \mathrm{E}+03$ & $1.17 \mathrm{E}+05$ & $3.24 \mathrm{E}+02$ & $8.36 \mathrm{E}+03$ & $1.65 \mathrm{E}+02$ \\
B17N66 Fine & $1.15 \mathrm{E}+03$ & $2.15 \mathrm{E}+04$ & $1.22 \mathrm{E}+03$ & $1.20 \mathrm{E}+05$ & $3.24 \mathrm{E}+02$ & $8.86 \mathrm{E}+03$ & $1.77 \mathrm{E}+02$ \\
\hline B1HK27 & $5.55 \mathrm{E}+02$ & $2.07 \mathrm{E}+04$ & $7.48 \mathrm{E}+02$ & $1.79 \mathrm{E}+05$ & $3.53 \mathrm{E}+02$ & $4.23 \mathrm{E}+03$ & $9.19 \mathrm{E}+01$ \\
B1HK27 Fine & $8.44 \mathrm{E}+02$ & $1.67 \mathrm{E}+04$ & $1.87 \mathrm{E}+03$ & $1.61 \mathrm{E}+05$ & $2.78 \mathrm{E}+02$ & $4.42 \mathrm{E}+03$ & $<8.49 \mathrm{E}+01$ \\
B1HK32 & $3.91 \mathrm{E}+02$ & $1.90 \mathrm{E}+04$ & $1.06 \mathrm{E}+03$ & $1.46 \mathrm{E}+05$ & $3.67 \mathrm{E}+02$ & $2.89 \mathrm{E}+03$ & $<7.07 \mathrm{E}+01$ \\
B1HK32 Fine & $7.26 \mathrm{E}+02$ & $1.53 \mathrm{E}+04$ & $2.38 \mathrm{E}+03$ & $1.38 \mathrm{E}+05$ & $2.68 \mathrm{E}+02$ & $4.88 \mathrm{E}+03$ & $8.72 \mathrm{E}+01$ \\
B1HK52 & $5.25 \mathrm{E}+02$ & $2.12 \mathrm{E}+04$ & $6.99 \mathrm{E}+02$ & $1.69 \mathrm{E}+05$ & $3.64 \mathrm{E}+02$ & $3.19 \mathrm{E}+03$ & $<6.93 \mathrm{E}+01$ \\
B1HK52 Fine & $8.02 \mathrm{E}+02$ & $2.19 \mathrm{E}+04$ & $1.24 \mathrm{E}+03$ & $1.44 \mathrm{E}+05$ & $3.53 \mathrm{E}+02$ & $5.52 \mathrm{E}+03$ & $9.40 \mathrm{E}+01$ \\
B1HK42 & $6.17 \mathrm{E}+02$ & $2.02 \mathrm{E}+04$ & $7.25 \mathrm{E}+02$ & $1.27 \mathrm{E}+05$ & $3.76 \mathrm{E}+02$ & $3.44 \mathrm{E}+03$ & $6.32 \mathrm{E}+01$ \\
B1HK42 Fine & $8.34 \mathrm{E}+02$ & $2.06 \mathrm{E}+04$ & $1.33 \mathrm{E}+03$ & $2.18 \mathrm{E}+05$ & $3.83 \mathrm{E}+02$ & $5.12 \mathrm{E}+03$ & $8.40 \mathrm{E}+01$ \\
B1HK57 & $7.91 \mathrm{E}+02$ & $1.79 \mathrm{E}+04$ & $7.61 \mathrm{E}+02$ & $1.42 \mathrm{E}+05$ & $3.07 \mathrm{E}+02$ & $3.60 \mathrm{E}+03$ & $<7.41 \mathrm{E}+01$ \\
B1HK57 Fine & $8.88 \mathrm{E}+02$ & $1.69 \mathrm{E}+04$ & $8.91 \mathrm{E}+02$ & $1.45 \mathrm{E}+05$ & $2.80 \mathrm{E}+02$ & $4.11 \mathrm{E}+03$ & $<7.60 \mathrm{E}+01$ \\
\hline
\end{tabular}

\subsubsection{Plutonium and Americium in Acid Digestions}

Results for ${ }^{239} \mathrm{Pu}$ and ${ }^{241} \mathrm{Am}$, as determined by ICP-MS, are shown in Table 3.6. Results in terms of $\mu \mathrm{g} / \mathrm{g}$-dry sediment are shown on the left-hand side of the table, and results in terms of pCi/g-dry sediment are shown on the right-hand side of the table. Results measured above the quantification limit are shown in red. Only five sediment samples had detectable ${ }^{239} \mathrm{Pu}$ concentration (B17RF2 C4, B17RF2 C1, B1HK27, and B1HK32, B1HK52 [fine only]). None of the samples had concentrations of ${ }^{241} \mathrm{Am}$ that were detectable by ICP-MS. Two samples (B1HK27 and B1HK32) that were above the detection limit can be compared with data from previous analyses (DOE/RL 2007). The ${ }^{239} \mathrm{Pu}$ results reported in Table 3.6 for sample B1HK27 and B1HK32 (91,600 pCi/g and 231,000 pCi/g, respectively) compare well with results reported in DOE/RL (2007) for these samples $(84,100 \mathrm{pCi} / \mathrm{g}$ and 254,000 pCi/g, respectively).

For several samples (B17RF2 C4, B1HK27, and B1HK32), ${ }^{239} \mathrm{Pu}$ concentrations are significantly elevated in the less than sand-size fraction relative to the bulk sediment. For these three sediment samples, concentrations are increased by a factor of 2.7, 19.1 and 4.5, respectively, compared to the bulk sediment. These three samples have relatively low less than sand-size fractions, ranging from $2.6 \%$ to $4.5 \%$ by weight. 
Table 3.6. Pu and Am Concentrations in Sediments Determined by ICP-MS Analysis of Acid Digests

\begin{tabular}{|c|c|c|c|c|}
\hline \multirow{3}{*}{$\frac{\text { Sample ID }}{\text { B17RF2 C4 }}$} & ${ }^{239} \mathrm{Pu}$ & ${ }^{241} \mathrm{Am}$ & ${ }^{239} \mathrm{Pu}$ & ${ }^{241} \mathrm{Am}$ \\
\hline & \multicolumn{2}{|c|}{$\mu \mathrm{g} / \mathrm{g}$-dry sediment } & \multicolumn{2}{|c|}{ pCi/g-dry sediment } \\
\hline & $1.04 \mathrm{E}+00$ & $<6.47 \mathrm{E}-01$ & $6.48 \mathrm{E}+04$ & $<2.23 \mathrm{E}+06$ \\
\hline B17RF2 C4 Fine & $2.82 \mathrm{E}+00$ & $<1.38 \mathrm{E}+00$ & $1.76 \mathrm{E}+05$ & $<4.75 \mathrm{E}+06$ \\
\hline B17RF2 C1 & 7.85E-01 & $<5.59 \mathrm{E}-01$ & $4.89 \mathrm{E}+04$ & $<1.92 \mathrm{E}+06$ \\
\hline B17N54 & $<7.20 \mathrm{E}-01$ & $<6.23 \mathrm{E}-01$ & $<4.49 \mathrm{E}+04$ & $<2.14 \mathrm{E}+06$ \\
\hline B17N54 Fine & $<8.93 \mathrm{E}-01$ & $<7.73 \mathrm{E}-01$ & $<5.56 \mathrm{E}+04$ & $<2.66 \mathrm{E}+06$ \\
\hline B17N59 & $<6.74 \mathrm{E}-01$ & $<5.83 \mathrm{E}-01$ & $<4.20 \mathrm{E}+04$ & $<2.01 \mathrm{E}+06$ \\
\hline B17N59 Fine & $<1.46 \mathrm{E}+00$ & $<1.26 \mathrm{E}+00$ & $<9.10 \mathrm{E}+04$ & $<4.34 \mathrm{E}+06$ \\
\hline B17N66 & $<5.37 \mathrm{E}-01$ & $<4.65 \mathrm{E}-01$ & $<3.35 \mathrm{E}+04$ & $<1.60 \mathrm{E}+06$ \\
\hline B17N66 Fine & $<6.69 \mathrm{E}-01$ & $<5.78 \mathrm{E}-01$ & $<4.17 \mathrm{E}+04$ & $<1.99 \mathrm{E}+06$ \\
\hline B1HK27 & $1.47 \mathrm{E}+00$ & $<5.43 \mathrm{E}-01$ & $9.16 \mathrm{E}+04$ & $<1.87 \mathrm{E}+06$ \\
\hline B1HK27 Fine & $2.81 \mathrm{E}+01$ & $<6.43 \mathrm{E}-01$ & $1.75 \mathrm{E}+06$ & $<2.21 \mathrm{E}+06$ \\
\hline В1НK32 & $3.70 \mathrm{E}+00$ & $<5.35 \mathrm{E}-01$ & $2.31 \mathrm{E}+05$ & $<1.84 \mathrm{E}+06$ \\
\hline B1HK32 Fine & $1.66 \mathrm{E}+01$ & $<5.32 \mathrm{E}-01$ & $1.03 E+06$ & $<1.83 \mathrm{E}+06$ \\
\hline B1HK52 & $<6.06 \mathrm{E}-01$ & $<5.25 \mathrm{E}-01$ & $<3.78 \mathrm{E}+04$ & $<1.81 \mathrm{E}+06$ \\
\hline B1HK52 Fine & 7.47E-01 & $<5.08 \mathrm{E}-01$ & $4.65 \mathrm{E}+04$ & $<1.75 \mathrm{E}+06$ \\
\hline B1HK42 & $<5.22 \mathrm{E}-01$ & $<4.52 \mathrm{E}-01$ & $<3.25 \mathrm{E}+04$ & $<1.56 \mathrm{E}+06$ \\
\hline B1HK42 Fine & $<6.67 \mathrm{E}-01$ & $<5.77 \mathrm{E}-01$ & $<4.16 \mathrm{E}+04$ & $<1.99 \mathrm{E}+06$ \\
\hline B1HK57 & $<6.48 \mathrm{E}-01$ & $<5.61 \mathrm{E}-01$ & $<4.04 \mathrm{E}+04$ & $<1.93 \mathrm{E}+06$ \\
\hline B1HK57 Fine & $<6.65 \mathrm{E}-01$ & $<5.75 \mathrm{E}-01$ & $<4.14 \mathrm{E}+04$ & $<1.98 \mathrm{E}+06$ \\
\hline
\end{tabular}

\subsection{Sediment Extract Results}

\subsubsection{Alkalinity and pH in Sediment Extracts}

Results for alkalinity and $\mathrm{pH}$ in the sediment extracts are shown in Table 3.7. For most of the samples, the alkalinity and $\mathrm{pH}$ values are similar in the groundwater prior to contact with the sediments (Table 2.2). Samples B17N54 and B1HK52 have undetectable alkalinity and low pH values, indicating these sample have had significant impacts from acidic waste solutions that were disposed in the 216-Z-9 Trench. These samples are indicated by shading. Shading was also added to these samples for results shown in Tables 3.3, and 3.8 through 3.11 to emphasis sediment extract results that have been impacted by contact with acidic waste. The B17RF2 C1 30-day contact extraction also indicates undetectable alkalinity and low pH values. The B17RF2 C1 1-day contact does not appear to be impacted by acidity. The reason for the significant differences observed for B17RF2 between the 1-day and 30-day contact is not clear. Sample heterogeneities could possibly account for the more acidic nature of B17RF2 30-day contact, or the longer equilibration period may have allowed for more complete reaction of the available acid with the alkalinity in the system. 
Table 3.7. $\mathrm{pH}$ and Alkalinity of Sediment Extracts

\begin{tabular}{cccc}
\hline Sample ID & Contact Time & Alkalinity & $\mathrm{pH}$ \\
\hline B17RF2 C4 & 1 day & 50.9 & 8.18 \\
B17RF2 C4 & 30 day & 116 & 7.91 \\
B17RF2 C1 & 1 day & 97.3 & 7.95 \\
B17RF2 C1 & 30 day & $<23.5$ & 6.90 \\
B17N54 & 1 day & $<23.5$ & 4.44 \\
B17N54 & 30 day & $<23.5$ & 4.34 \\
B17N59 & 1 day & 84.4 & 7.77 \\
B17N59 & 30 day & 85.9 & 7.78 \\
B17N66 & 1 day & 157 & 8.28 \\
B17N66 & 30 day & 155 & 8.21 \\
\hline B1HK27 & 1 day & 67.6 & 7.68 \\
B1HK27 & 30 day & 82.1 & 7.85 \\
B1HK32 & 1 day & 80.6 & 7.31 \\
B1HK32 & 30 day & 129 & 7.88 \\
B1HK52 & 1 day & $<23.5$ & 5.40 \\
B1HK52 & 30 day & $<23.5$ & 4.55 \\
B1HK42 & 1 day & 99.6 & 8.02 \\
B1HK42 & 30 day & 119 & 8.07 \\
B1HK57 & 1 day & 130 & 8.14 \\
B1HK57 & 30 day & 125 & 8.12 \\
\hline
\end{tabular}

\subsubsection{Anions in Sediment Extracts}

Anion concentrations in the sediment extracts are shown in Table 3.8, along with the calculated concentration extracted from the sediments. Only anions determined at concentrations above the quantification limit are shown. Fluoride, phosphate, and nitrite were below their respective quantification limits in all samples. The quantification limits for fluoride, phosphate, and nitrite were $20 \mu \mathrm{g} / \mathrm{mL}$, $150 \mu \mathrm{g} / \mathrm{mL}$, and $100 \mu \mathrm{g} / \mathrm{mL}$, respectively. These high quantification limits were the result of large dilutions required due to the very high nitrate concentrations in some samples. The high nitrate results from samples B17N54, B17N59, and B17N66 from well 299-W15-46, and sample B1HK52 from well 299-W15-46, indicate these samples were significantly impacted by aqueous waste solutions containing high nitrate concentrations. The high acidity observed in the extracts from samples B17N54 and B1HK52 (Table 3.7) indicate these high nitrate waste solutions were also acidic. The high carbonate content that occurs in sediment sample B17N59 appears to have been effective at neutralizing this acidity and preventing acidic conditions below this depth. The two samples acidic samples (B17N54 and B1HK52) also have relatively high chloride concentrations. This may have resulted from accelerated degradation of carbon tetrachloride under acidic conditions. Acidic solutions in contact with Hanford sediments could release ferrous iron which could result in surface mediated reductive dehalogenation of carbon tetrachloride by surface adsorbed ferrous iron (Elsner et al. 2003). These data are used as input data for the geochemical modeling discussed in Section 4.0. 
Table 3.8. Major Anion Concentrations in Sediment Extracts Determined by IC and Extracted Concentration in the Sediments

\begin{tabular}{|c|c|c|c|c|c|c|c|}
\hline \multirow[b]{2}{*}{ Sample ID } & \multirow{2}{*}{$\begin{array}{c}\text { Contact } \\
\text { Time }\end{array}$} & $\mathrm{Cl}^{-}$ & $\mathrm{NO}_{3}^{-}$ & $\mathrm{SO}_{4}{ }^{2-}$ & $\mathrm{Cl}^{-}$ & $\mathrm{NO}_{3}^{-}$ & $\mathrm{SO}_{4}{ }^{2-}$ \\
\hline & & \multicolumn{3}{|c|}{$\mu \mathrm{g} / \mathrm{mL}$} & \multicolumn{3}{|c|}{$\mu g / g$-dry sediment } \\
\hline B17RF2 C4 & 1 day & $<5.00 \mathrm{E}+01$ & $1.73 E+02$ & $2.83 \mathrm{E}+02$ & $<6.12 \mathrm{E}+01$ & $2.12 \mathrm{E}+02$ & $3.47 \mathrm{E}+02$ \\
\hline B17RF2 C4 & 30 day & $<5.00 \mathrm{E}+01$ & $1.54 \mathrm{E}+02$ & $3.25 E+02$ & $<6.05 \mathrm{E}+01$ & $1.87 \mathrm{E}+02$ & $3.93 \mathrm{E}+02$ \\
\hline B17RF2 C1 & 1 day & $<5.00 \mathrm{E}+01$ & $2.77 \mathrm{E}+02$ & $3.47 \mathrm{E}+02$ & $<5.36 \mathrm{E}+01$ & $2.97 \mathrm{E}+02$ & $3.72 E+02$ \\
\hline B17RF2 C1 & 30 day & $5.00 \mathrm{E}+01$ & $4.64 \mathrm{E}+02$ & $3.51 \mathrm{E}+02$ & $5.41 \mathrm{E}+01$ & $5.02 E+02$ & $3.80 \mathrm{E}+02$ \\
\hline B17N54 & 1 day & $5.61 \mathrm{E}+01$ & $4.53 \mathrm{E}+03$ & $<1.50 \mathrm{E}+02$ & $6.27 \mathrm{E}+01$ & $5.06 \mathrm{E}+03$ & $<1.68 \mathrm{E}+02$ \\
\hline B17N54 & 30 day & $5.98 \mathrm{E}+01$ & $4.77 \mathrm{E}+03$ & $<1.50 \mathrm{E}+02$ & $6.91 \mathrm{E}+01$ & $5.51 \mathrm{E}+03$ & $<1.73 \mathrm{E}+02$ \\
\hline B17N59 & 1 day & $<5.00 \mathrm{E}+01$ & $3.47 \mathrm{E}+03$ & $<1.50 \mathrm{E}+02$ & $<6.48 \mathrm{E}+01$ & $4.49 \mathrm{E}+03$ & $<1.94 \mathrm{E}+02$ \\
\hline B17N59 & 30 day & $<5.00 \mathrm{E}+01$ & $3.22 \mathrm{E}+03$ & $<1.50 \mathrm{E}+02$ & $<6.55 \mathrm{E}+01$ & $4.23 \mathrm{E}+03$ & $<1.97 \mathrm{E}+02$ \\
\hline B17N66 & 1 day & $<5.00 \mathrm{E}+01$ & $1.02 \mathrm{E}+03$ & $<1.50 \mathrm{E}+02$ & $<5.36 \mathrm{E}+01$ & $1.09 \mathrm{E}+03$ & $<1.61 \mathrm{E}+02$ \\
\hline B17N66 & 30 day & $<5.00 \mathrm{E}+01$ & $1.17 \mathrm{E}+03$ & $<1.50 \mathrm{E}+02$ & $<5.27 \mathrm{E}+01$ & $1.23 \mathrm{E}+03$ & $<1.58 \mathrm{E}+02$ \\
\hline B1HK27 & 1 day & $<5.00 \mathrm{E}+01$ & $<1.00 \mathrm{E}+02$ & $2.78 \mathrm{E}+02$ & $<5.39 \mathrm{E}+01$ & $<1.08 \mathrm{E}+02$ & $3.00 \mathrm{E}+02$ \\
\hline B1HK27 & 30 day & $<5.00 \mathrm{E}+01$ & $<1.00 \mathrm{E}+02$ & $3.23 \mathrm{E}+02$ & $<5.38 \mathrm{E}+01$ & $<1.08 \mathrm{E}+02$ & $3.47 \mathrm{E}+02$ \\
\hline В1НК32 & 1 day & $9.60 \mathrm{E}+01$ & $<1.00 \mathrm{E}+02$ & $2.11 \mathrm{E}+02$ & $1.02 \mathrm{E}+02$ & $<1.07 \mathrm{E}+02$ & $2.25 \mathrm{E}+02$ \\
\hline B1HK32 & 30 day & $9.57 \mathrm{E}+01$ & $<1.00 \mathrm{E}+02$ & $2.38 \mathrm{E}+02$ & $1.15 \mathrm{E}+02$ & $<1.20 \mathrm{E}+02$ & $2.86 \mathrm{E}+02$ \\
\hline B1HK52 & 1 day & $1.34 \mathrm{E}+02$ & $5.05 E+03$ & $1.60 \mathrm{E}+02$ & $1.53 \mathrm{E}+02$ & $5.76 \mathrm{E}+03$ & $1.82 \mathrm{E}+02$ \\
\hline В1НК52 & 30 day & $1.32 \mathrm{E}+02$ & $5.28 \mathrm{E}+03$ & $<1.50 \mathrm{E}+02$ & $1.49 \mathrm{E}+02$ & $5.96 \mathrm{E}+03$ & $<1.69 \mathrm{E}+02$ \\
\hline B1HK42 & 1 day & $<5.00 \mathrm{E}+01$ & $4.52 \mathrm{E}+02$ & $<1.50 \mathrm{E}+02$ & $<5.70 \mathrm{E}+01$ & $5.15 E+02$ & $<1.71 \mathrm{E}+02$ \\
\hline B1HK42 & 30 day & $<5.00 \mathrm{E}+01$ & $5.66 \mathrm{E}+02$ & $<1.50 \mathrm{E}+02$ & $<5.68 \mathrm{E}+01$ & $6.43 \mathrm{E}+02$ & $<1.70 \mathrm{E}+02$ \\
\hline B1HK57 & 1 day & $<5.00 \mathrm{E}+01$ & $6.62 \mathrm{E}+02$ & $<1.50 \mathrm{E}+02$ & $<5.37 \mathrm{E}+01$ & $7.10 \mathrm{E}+02$ & $<1.61 \mathrm{E}+02$ \\
\hline B1HK57 & 30 day & $<5.00 \mathrm{E}+01$ & $7.46 \mathrm{E}+02$ & $1.57 \mathrm{E}+02$ & $<5.13 \mathrm{E}+01$ & $7.65 \mathrm{E}+02$ & $1.61 \mathrm{E}+02$ \\
\hline
\end{tabular}

\subsubsection{Elemental Analysis of Sediment Extracts}

Tables 3.9 and 3.10 contain dissolved element concentrations in groundwater extracts as determined by ICP-OES. Only elements with a concentration result above the quantification limit are tabulated. These data are used as input data for the geochemical modeling discussed in Section 4.0. Note that sample extracts containing relatively high sodium are the same samples that contained high nitrate concentrations (B17N54, B17N59, B17N66, and B1HK52). These results suggest these samples were impacted by acidic aqueous wastes containing high concentrations of $\mathrm{NaNO}_{3}$. In addition the highest metal concentrations are found in the two samples that were most impacted by acidic wastes (B17N54 and B1HK52). 
Table 3.9. Element Concentrations in Sediment Extracts Determined by ICP-OES (Al through Mg)

\begin{tabular}{|c|c|c|c|c|c|c|c|c|}
\hline \multirow[b]{2}{*}{ Sample ID } & \multirow{2}{*}{$\begin{array}{c}\text { Contact } \\
\text { Time }\end{array}$} & $\mathrm{Al}$ & $\mathrm{Ba}$ & Ca & $\mathrm{Cd}$ & Co & $\mathrm{K}$ & $\mathrm{Mg}$ \\
\hline & & \multicolumn{7}{|c|}{$\mu \mathrm{g} / \mathrm{L}$} \\
\hline B17RF2 C4 & 1 day & $<5.72 \mathrm{E}+02$ & $<5.86 \mathrm{E}+01$ & $9.17 \mathrm{E}+04$ & $<1.79 \mathrm{E}+02$ & $<6.40 \mathrm{E}+02$ & $<1.55 \mathrm{E}+04$ & $2.51 E+04$ \\
\hline B17RF2 C4 & 30 day & $<5.72 \mathrm{E}+02$ & $<5.86 \mathrm{E}+01$ & $1.14 \mathrm{E}+05$ & $<1.79 \mathrm{E}+02$ & $<6.40 \mathrm{E}+02$ & $<1.55 \mathrm{E}+04$ & $2.78 E+04$ \\
\hline B17RF2 C1 & 1 day & $<5.72 \mathrm{E}+02$ & $<5.86 \mathrm{E}+01$ & $1.25 \mathrm{E}+05$ & $<1.79 \mathrm{E}+02$ & $<6.40 \mathrm{E}+02$ & $<1.55 \mathrm{E}+04$ & $3.25 \mathrm{E}+04$ \\
\hline B17RF2 C1 & 30 day & $<5.72 \mathrm{E}+02$ & $<5.86 \mathrm{E}+01$ & $1.02 \mathrm{E}+05$ & $<1.79 \mathrm{E}+02$ & $<6.40 \mathrm{E}+02$ & $<1.55 \mathrm{E}+04$ & $4.35 \mathrm{E}+04$ \\
\hline B17N54 & 1 day & $6.06 \mathrm{E}+03$ & $6.77 \mathrm{E}+02$ & $6.27 E+05$ & $1.40 \mathrm{E}+03$ & $<6.40 \mathrm{E}+02$ & $2.14 \mathrm{E}+04$ & $1.46 \mathrm{E}+05$ \\
\hline B17N54 & 30 day & $1.07 \mathrm{E}+04$ & $4.95 \mathrm{E}+02$ & $6.81 \mathrm{E}+05$ & $1.57 \mathrm{E}+03$ & $<6.40 \mathrm{E}+02$ & $2.35 E+04$ & $1.59 \mathrm{E}+05$ \\
\hline B17N59 & 1 day & $<5.72 \mathrm{E}+02$ & $1.45 \mathrm{E}+02$ & $4.07 E+05$ & $1.91 \mathrm{E}+02$ & $<6.40 \mathrm{E}+02$ & $2.07 E+04$ & $9.34 \mathrm{E}+04$ \\
\hline B17N59 & 30 day & $<5.72 \mathrm{E}+02$ & $1.18 \mathrm{E}+02$ & $3.48 \mathrm{E}+05$ & $2.14 \mathrm{E}+02$ & $<6.40 \mathrm{E}+02$ & $1.81 \mathrm{E}+04$ & $8.92 \mathrm{E}+04$ \\
\hline B17N66 & 1 day & $<5.72 \mathrm{E}+02$ & $6.39 \mathrm{E}+01$ & $7.26 \mathrm{E}+04$ & $<1.79 \mathrm{E}+02$ & $<6.40 \mathrm{E}+02$ & $<1.55 \mathrm{E}+04$ & $2.75 E+04$ \\
\hline B17N66 & 30 day & $<5.72 \mathrm{E}+02$ & $7.46 \mathrm{E}+01$ & $7.34 \mathrm{E}+04$ & $<1.79 \mathrm{E}+02$ & $<6.40 \mathrm{E}+02$ & $<1.55 \mathrm{E}+04$ & $2.80 \mathrm{E}+04$ \\
\hline B1HK27 & 1 day & $<2.86 \mathrm{E}+03$ & $<2.93 \mathrm{E}+02$ & $9.17 E+04$ & $<8.96 \mathrm{E}+02$ & $<3.20 \mathrm{E}+03$ & $<7.76 \mathrm{E}+04$ & $2.02 \mathrm{E}+04$ \\
\hline B1HK27 & 30 day & $<5.72 \mathrm{E}+02$ & $<5.86 \mathrm{E}+01$ & $1.01 \mathrm{E}+05$ & $<1.79 \mathrm{E}+02$ & $<6.40 \mathrm{E}+02$ & $<1.55 \mathrm{E}+04$ & $2.09 \mathrm{E}+04$ \\
\hline B1HK32 & 1 day & $<5.72 \mathrm{E}+02$ & $<5.86 \mathrm{E}+01$ & $9.39 \mathrm{E}+04$ & $<1.79 \mathrm{E}+02$ & $<6.40 \mathrm{E}+02$ & $<1.55 \mathrm{E}+04$ & $1.85 \mathrm{E}+04$ \\
\hline B1HK32 & 30 day & $<5.72 \mathrm{E}+02$ & $<5.86 \mathrm{E}+01$ & $1.20 \mathrm{E}+05$ & $<1.79 \mathrm{E}+02$ & $<6.40 \mathrm{E}+02$ & $<1.55 \mathrm{E}+04$ & $2.05 \mathrm{E}+04$ \\
\hline B1HK52 & & $<5.72 \mathrm{E}+02$ & & & $3.56 \mathrm{E}+02$ & $<6.40 \mathrm{E}+02$ & $7.85 E+04$ & $1.79 \mathrm{E}+05$ \\
\hline B1HK52 & 30 day & $1.63 \mathrm{E}+03$ & $4.75 \mathrm{E}+02$ & $4.49 \mathrm{E}+05$ & $1.31 \mathrm{E}+03$ & $7.35 \mathrm{E}+02$ & $9.04 \mathrm{E}+04$ & $2.20 \mathrm{E}+05$ \\
\hline B1HK42 & 1 day & $<5.72 \mathrm{E}+02$ & $<5.86 \mathrm{E}+01$ & $1.39 \mathrm{E}+05$ & $<1.79 \mathrm{E}+02$ & $<6.40 \mathrm{E}+02$ & $<1.55 \mathrm{E}+04$ & $1.65 \mathrm{E}+04$ \\
\hline B1HK42 & 30 day & $<5.72 \mathrm{E}+02$ & $<5.86 \mathrm{E}+01$ & $1.65 \mathrm{E}+05$ & $<1.79 \mathrm{E}+02$ & $<6.40 \mathrm{E}+02$ & $<1.55 \mathrm{E}+04$ & $1.95 \mathrm{E}+04$ \\
\hline B1HK57 & 1 day & $<5.72 \mathrm{E}+02$ & $<5.86 \mathrm{E}+01$ & $1.33 \mathrm{E}+05$ & $<1.79 \mathrm{E}+02$ & $<6.40 \mathrm{E}+02$ & $2.98 \mathrm{E}+04$ & $1.52 \mathrm{E}+04$ \\
\hline B1HK57 & 30 day & $<5.72 \mathrm{E}+02$ & $<5.86 \mathrm{E}+01$ & $1.32 \mathrm{E}+05$ & $<1.79 \mathrm{E}+02$ & $<6.40 \mathrm{E}+02$ & $2.99 \mathrm{E}+04$ & $1.60 \mathrm{E}+04$ \\
\hline
\end{tabular}

Table 3.10. Element Concentrations in Sediment Extracts Determined by ICP-OES (Mn through Sr)

\begin{tabular}{cccccccc}
\hline & Contact & $\mathrm{Mn}$ & $\mathrm{Na}$ & $\mathrm{P}$ & $\mathrm{S}$ & $\mathrm{Si}$ & $\mathrm{Sr}$ \\
\cline { 3 - 8 } Sample ID & Time & \multicolumn{7}{c}{$\mu \mathrm{g} / \mathrm{L}$} \\
\hline B17RF2 C4 & 1 day & $4.30 \mathrm{E}+02$ & $1.14 \mathrm{E}+05$ & $<6.88 \mathrm{E}+03$ & $9.42 \mathrm{E}+04$ & $<1.00 \mathrm{E}+04$ & $<3.48 \mathrm{E}+02$ \\
B17RF2 C4 & 30 day & $7.95 \mathrm{E}+02$ & $1.00 \mathrm{E}+05$ & $<6.88 \mathrm{E}+03$ & $1.15 \mathrm{E}+05$ & $<1.00 \mathrm{E}+04$ & $<3.48 \mathrm{E}+02$ \\
B17RF2 C1 & 1 day & $6.87 \mathrm{E}+02$ & $1.14 \mathrm{E}+05$ & $<6.88 \mathrm{E}+03$ & $1.17 \mathrm{E}+05$ & $<1.00 \mathrm{E}+04$ & $<3.48 \mathrm{E}+02$ \\
B17RF2 C1 & 30 day & $2.07 \mathrm{E}+03$ & $1.57 \mathrm{E}+05$ & $<6.88 \mathrm{E}+03$ & $1.21 \mathrm{E}+05$ & $<1.00 \mathrm{E}+04$ & $<3.48 \mathrm{E}+02$ \\
B17N54 & 1 day & $1.17 \mathrm{E}+04$ & $6.53 \mathrm{E}+05$ & $<6.88 \mathrm{E}+03$ & $<2.05 \mathrm{E}+04$ & $<1.00 \mathrm{E}+04$ & $1.65 \mathrm{E}+03$ \\
B17N54 & 30 day & $1.29 \mathrm{E}+04$ & $7.09 \mathrm{E}+05$ & $<6.88 \mathrm{E}+03$ & $<2.05 \mathrm{E}+04$ & $<1.00 \mathrm{E}+04$ & $1.74 \mathrm{E}+03$ \\
B17N59 & 1 day & $2.88 \mathrm{E}+03$ & $7.67 \mathrm{E}+05$ & $<6.88 \mathrm{E}+03$ & $<2.05 \mathrm{E}+04$ & $<1.00 \mathrm{E}+04$ & $8.91 \mathrm{E}+02$ \\
B17N59 & 30 day & $3.08 \mathrm{E}+03$ & $7.10 \mathrm{E}+05$ & $<6.88 \mathrm{E}+03$ & $<2.05 \mathrm{E}+04$ & $<1.00 \mathrm{E}+04$ & $7.32 \mathrm{E}+02$ \\
B17N66 & 1 day & $<1.14 \mathrm{E}+02$ & $3.68 \mathrm{E}+05$ & $<6.88 \mathrm{E}+03$ & $3.07 \mathrm{E}+04$ & $2.15 \mathrm{E}+04$ & $<3.48 \mathrm{E}+02$ \\
B17N66 & 30 day & $<1.14 \mathrm{E}+02$ & $4.32 \mathrm{E}+05$ & $<6.88 \mathrm{E}+03$ & $3.59 \mathrm{E}+04$ & $1.75 \mathrm{E}+04$ & $<3.48 \mathrm{E}+02$ \\
\hline B1HK27 & 1 day & $8.83 \mathrm{E}+02$ & $4.46 \mathrm{E}+04$ & $<3.44 \mathrm{E}+04$ & $<1.03 \mathrm{E}+05$ & $<5.00 \mathrm{E}+04$ & $<1.74 \mathrm{E}+03$ \\
B1HK27 & 30 day & $1.07 \mathrm{E}+03$ & $4.98 \mathrm{E}+04$ & $9.21 \mathrm{E}+03$ & $1.11 \mathrm{E}+05$ & $<1.00 \mathrm{E}+04$ & $<3.48 \mathrm{E}+02$ \\
B1HK32 & 1 day & $8.87 \mathrm{E}+02$ & $7.84 \mathrm{E}+04$ & $3.02 \mathrm{E}+04$ & $7.34 \mathrm{E}+04$ & $<1.00 \mathrm{E}+04$ & $<3.48 \mathrm{E}+02$ \\
B1HK32 & 30 day & $9.11 \mathrm{E}+02$ & $7.73 \mathrm{E}+04$ & $3.00 \mathrm{E}+04$ & $7.84 \mathrm{E}+04$ & $<1.00 \mathrm{E}+04$ & $<3.48 \mathrm{E}+02$ \\
B1HK52 & 1 day & $4.80 \mathrm{E}+03$ & $1.14 \mathrm{E}+06$ & $<6.88 \mathrm{E}+03$ & $4.88 \mathrm{E}+04$ & $<1.00 \mathrm{E}+04$ & $1.98 \mathrm{E}+03$ \\
B1HK52 & 30 day & $3.95 \mathrm{E}+04$ & $1.02 \mathrm{E}+06$ & $<6.88 \mathrm{E}+03$ & $<2.05 \mathrm{E}+04$ & $<1.00 \mathrm{E}+04$ & $1.87 \mathrm{E}+03$ \\
B1HK42 & 1 day & $<1.14 \mathrm{E}+02$ & $8.87 \mathrm{E}+04$ & $<6.88 \mathrm{E}+03$ & $4.20 \mathrm{E}+04$ & $<1.00 \mathrm{E}+04$ & $4.02 \mathrm{E}+02$ \\
B1HK42 & 30 day & $<1.14 \mathrm{E}+02$ & $1.04 \mathrm{E}+05$ & $<6.88 \mathrm{E}+03$ & $4.25 \mathrm{E}+04$ & $<1.00 \mathrm{E}+04$ & $4.67 \mathrm{E}+02$ \\
B1HK57 & 1 day & $<1.14 \mathrm{E}+02$ & $2.00 \mathrm{E}+05$ & $<6.88 \mathrm{E}+03$ & $4.15 \mathrm{E}+04$ & $<1.00 \mathrm{E}+04$ & $<3.48 \mathrm{E}+02$ \\
B1HK57 & 30 day & $<1.14 \mathrm{E}+02$ & $2.41 \mathrm{E}+05$ & $<6.88 \mathrm{E}+03$ & $5.37 \mathrm{E}+04$ & $<1.00 \mathrm{E}+04$ & $<3.48 \mathrm{E}+02$ \\
\hline
\end{tabular}




\subsubsection{Plutonium and Americium in Sediment Extracts}

Plutonium and americium concentrations in sediment extracts determined by ICP-MS and the concentrations extracted from the sediment are shown in Table 3.11. Only two sediment samples had measurable ${ }^{239} \mathrm{Pu}$ or ${ }^{241} \mathrm{Am}$ in their sediment extracts as determined by ICP-MS. These samples are indicated by shading in Table 3.11. For sample B1HK52, ${ }^{239} \mathrm{Pu}$ was measured at a concentration of 0.148 $\mu \mathrm{g} / \mathrm{L}(922 \mathrm{pCi} / \mathrm{L})$ in the 1-day contact sediment extract. For sample B17N54, ${ }^{241} \mathrm{Am}$ was measured in both the 1-day $\left(0.833 \mu \mathrm{g} / \mathrm{L}\right.$ or $\left.2.87 \times 10^{6} \mathrm{pCi} / \mathrm{L}\right)$ and 30 -day $\left(0.795 \mu \mathrm{g} / \mathrm{L}\right.$ or $\left.2.74 \times 10^{6} \mathrm{pCi} / \mathrm{L}\right)$ contact sediment extracts and in the 30-day contact sediment extract of sample B1HK52 $(0.162 \mu \mathrm{g} / \mathrm{L}$ or $5.57 \mathrm{x}$ $10^{6} \mathrm{pCi} / \mathrm{L}$ ). The percentages of ${ }^{239} \mathrm{Pu}$ or ${ }^{241} \mathrm{Am}$ that were released from the sediments were calculated from these data and total concentrations estimated from the acid extracts (Table 3.6). The calculated values in Table 3.11 are all indicated to be greater than values because the total concentrations were below the detection limit and the value used as the total was the quantification limit. These results indicate the percentages of ${ }^{239} \mathrm{Pu}$ and ${ }^{241} \mathrm{Am}$ that were released are small, $>0.03 \%$ for ${ }^{239} \mathrm{Pu}$ and $>0.04$ to $>0.15 \%$ for ${ }^{241} \mathrm{Am}$. All groundwater extract samples collected for the colloid mobility experiment were below the detection limit. This indicates significant concentrations of filterable $(>0.0018 \mu \mathrm{m})$ colloids do not form or enhance the mobility of ${ }^{239} \mathrm{Pu}$ and ${ }^{241} \mathrm{Am}$ in these samples. Note that very small colloidal particles or nanoclusters could potentially pass through these filters.

The sediment extracts that had measureable concentrations of plutonium or americium had concentrations that were considerable above the drinking water standard of $15 \mathrm{pCi} / \mathrm{L}$. The ICP-MS detection limit for ${ }^{239} \mathrm{Pu}$ was $7 \mathrm{pCi} / \mathrm{L}(1.14 \mathrm{E}-04 \mu \mathrm{g} / \mathrm{L})$ and $340 \mathrm{pCi} / \mathrm{L}(9.88 \mathrm{E}-05 \mu \mathrm{g} / \mathrm{L})$ for ${ }^{241} \mathrm{Am}$. The sediment extracts that had measurable concentrations of plutonium and americium were all acidic compared to typical groundwater (see Table 3.7), ranging from $\mathrm{pH} 4.34$ to 5.40. These results indicate that acidic conditions are required to mobilize plutonium and americium from vadose zone sediments impacted by 216-Z-9 Trench wastes. 
Table 3.11. Pu and Am Concentrations in Sediment Extracts Determined by ICP-MS and Extracted Concentration in the Sediments. Samples collected for the colloid study are indicated with (col).

\begin{tabular}{|c|c|c|c|c|c|c|c|}
\hline \multirow[b]{2}{*}{ Sample ID } & \multirow{2}{*}{$\begin{array}{c}\text { Contact } \\
\text { Time } \\
\end{array}$} & ${ }^{239} \mathrm{Pu}$ & ${ }^{241} \mathrm{Am}$ & ${ }^{239} \mathrm{Pu}$ & ${ }^{241} \mathrm{Am}$ & ${ }^{239} \mathrm{Pu}$ & ${ }^{241} \mathrm{Am}$ \\
\hline & & \multicolumn{2}{|c|}{$\mu \mathrm{g} / \mathrm{L}$} & \multicolumn{2}{|c|}{$\mu \mathrm{g} / \mathrm{g}$-dry sediment } & \multicolumn{2}{|c|}{ \% Released } \\
\hline B17RF2 C4 & 1 day & $<1.14 \mathrm{E}-04$ & $<9.88 \mathrm{E}-05$ & $<1.40 \mathrm{E}-04$ & $<1.21 \mathrm{E}-04$ & & \\
\hline B17RF2 C4 & 30 day & $<1.14 \mathrm{E}-04$ & $<9.88 \mathrm{E}-05$ & $<1.38 \mathrm{E}-04$ & $<1.19 \mathrm{E}-04$ & & \\
\hline B17RF2 C1 & 1 day & $<1.14 \mathrm{E}-04$ & $<9.88 \mathrm{E}-05$ & $<1.22 \mathrm{E}-04$ & $<1.06 \mathrm{E}-04$ & & \\
\hline B17RF2 C1 & 30 day & $<1.14 \mathrm{E}-04$ & $<9.88 \mathrm{E}-05$ & $<1.24 \mathrm{E}-04$ & $<1.07 \mathrm{E}-04$ & & \\
\hline B17RF2 C1 - 0.45 $\mu$ (col) & 1 day & $<1.14 \mathrm{E}-04$ & $<9.88 \mathrm{E}-05$ & $<5.91 \mathrm{E}-04$ & $<5.11 \mathrm{E}-04$ & & \\
\hline B17RF2 C1 $-0.0018 \mu(\mathrm{col})$ & 1 day & $<1.14 \mathrm{E}-04$ & $<9.88 \mathrm{E}-05$ & $<5.91 \mathrm{E}-04$ & $<5.11 \mathrm{E}-04$ & & \\
\hline B17N54 & 1 day & $<1.14 \mathrm{E}-04$ & 8.33E-01 & $<1.28 \mathrm{E}-04$ & $9.31 \mathrm{E}-04$ & & $>0.15$ \\
\hline B17N54 & 30 day & $<1.14 \mathrm{E}-04$ & 7.95E-01 & $<1.32 \mathrm{E}-04$ & $9.18 \mathrm{E}-04$ & & $>0.15$ \\
\hline B17N59 & 1 day & $<1.14 \mathrm{E}-04$ & $<9.88 \mathrm{E}-05$ & $<1.48 \mathrm{E}-04$ & $<1.28 \mathrm{E}-04$ & & \\
\hline B17N59 & 30 day & $<1.14 \mathrm{E}-04$ & $<9.88 \mathrm{E}-05$ & $<1.50 \mathrm{E}-04$ & $<1.30 \mathrm{E}-04$ & & \\
\hline B17N66 & 1 day & $<1.14 \mathrm{E}-04$ & $<9.88 \mathrm{E}-05$ & $<1.23 \mathrm{E}-04$ & $<1.06 \mathrm{E}-04$ & & \\
\hline B17N66 & 30 day & $<1.14 \mathrm{E}-04$ & $<9.88 \mathrm{E}-05$ & $<1.20 \mathrm{E}-04$ & $<1.04 \mathrm{E}-04$ & & \\
\hline B1HK27 & 1 day & $<1.14 \mathrm{E}-04$ & $<9.88 \mathrm{E}-05$ & $<1.23 \mathrm{E}-04$ & $<1.06 \mathrm{E}-04$ & & \\
\hline B1HK27 & 30 day & $<1.14 \mathrm{E}-04$ & $<9.88 \mathrm{E}-05$ & $<1.23 \mathrm{E}-04$ & $<1.06 \mathrm{E}-04$ & & \\
\hline B1HK32 & 1 day & $<1.14 \mathrm{E}-04$ & $<9.88 \mathrm{E}-05$ & $<1.22 \mathrm{E}-04$ & $<1.05 \mathrm{E}-04$ & & \\
\hline B1HK32 & 30 day & $<1.14 \mathrm{E}-04$ & $<9.88 \mathrm{E}-05$ & $<1.37 \mathrm{E}-04$ & $<1.19 \mathrm{E}-04$ & & \\
\hline B1HK32 - 0.45 $\mu(\mathrm{col})$ & 1 day & $<1.14 \mathrm{E}-04$ & $<9.88 \mathrm{E}-05$ & $<5.69 \mathrm{E}-04$ & $<4.92 \mathrm{E}-04$ & & \\
\hline B1HK32 $-0.0018 \mu(\mathrm{col})$ & 1 day & $<1.14 \mathrm{E}-04$ & $<9.88 \mathrm{E}-05$ & $<5.69 \mathrm{E}-04$ & $<4.92 \mathrm{E}-04$ & & \\
\hline B1HK52 & 1 day & $1.48 \mathrm{E}-01$ & $<9.88 \mathrm{E}-05$ & $1.68 \mathrm{E}-04$ & $<1.13 \mathrm{E}-04$ & $>0.03$ & \\
\hline B1HK52 & 30 day & $<1.14 \mathrm{E}-04$ & 1.62E-01 & $<1.29 \mathrm{E}-04$ & 1.83E-04 & & $>0.04$ \\
\hline B1HK42 & 1 day & $<1.14 \mathrm{E}-04$ & $<9.88 \mathrm{E}-05$ & $<1.30 \mathrm{E}-04$ & $<1.13 \mathrm{E}-04$ & & \\
\hline B1HK42 & 30 day & $<1.14 \mathrm{E}-04$ & $<9.88 \mathrm{E}-05$ & $<1.30 \mathrm{E}-04$ & $<1.12 \mathrm{E}-04$ & & \\
\hline B1HK42 - 0.45 $\mu$ (col) & 1 day & $<1.14 \mathrm{E}-04$ & $<9.88 \mathrm{E}-05$ & $<6.08 \mathrm{E}-04$ & $<5.26 \mathrm{E}-04$ & & \\
\hline B1HK42 $-0.0018 \mu(\mathrm{col})$ & 1 day & $<1.14 \mathrm{E}-04$ & $<9.88 \mathrm{E}-05$ & $<6.08 \mathrm{E}-04$ & $<5.26 \mathrm{E}-04$ & & \\
\hline B1HK57 & 1 day & $<1.14 \mathrm{E}-04$ & $<9.88 \mathrm{E}-05$ & $<1.23 \mathrm{E}-04$ & $<1.06 \mathrm{E}-04$ & & \\
\hline B1HK57 & 30 day & $<1.14 \mathrm{E}-04$ & $<9.88 \mathrm{E}-05$ & $<1.17 \mathrm{E}-04$ & $<1.01 \mathrm{E}-04$ & & \\
\hline
\end{tabular}




\subsection{Geochemical Modeling}

The React module of Geochemist’s Workbench ${ }^{\circledR}$ Version 7.0.3 (Bethke and Yeakel 2007) was used to calculate mineral saturation indices (SIs) and solution speciation based on measured compositions of leachates from the 1- and 30-day sediment extraction tests. The SI values were computed to identify the saturation state of solid phases that could potentially control plutonium and americium solubility in the leachate solutions. The SI is defined in Equation (4.1):

$$
\mathrm{SI}=\log \left(\mathrm{Q} / \mathrm{K}_{\mathrm{sp}}\right)
$$

where $\mathrm{Q}$ is the activity product and $\mathrm{K}_{\mathrm{sp}}$ is the mineral solubility product at equilibrium at the temperature of interest. Minerals with SI values near zero (within \pm 0.5 , SI values are unitless) are generally considered to be near equilibrium with the solution composition. More positive values are considered to be oversaturated, and more negative values are considered undersaturated with respect to the solution composition. The SI values for the groundwater extracts were calculated using the thermodynamic database file (thermo_NEA.dat) that is supplied with Geochemist's Workbench Version 7.0.3. This database is based upon data that were recently critically reviewed and selected by the Organization for Economic Co-operation and Development/Nuclear Energy Agency-Thermochemical Database Project (Lemire et al. 2001, Guillaumont et al. 2003).

To run these calculations for reduction-oxidation (redox) sensitive elements such as plutonium, it is necessary to know the redox potential or Eh. Eh measurements can be problematic because many elements with more than one oxidation state do not exhibit reversible behavior at the redox electrode surface, and some systems will give mixed potentials depending on the presence of several different couples (Grenthe et al. 1992, Lindberg and Runnels 1984). The approach used in this study was to assume the system was in equilibrium with atmospheric oxygen (oxygen fugacity set to 0.21 atmospheres). This is a reasonable assumption because the Hanford Site vadose zone is generally oxidizing and the sediment samples were exposed to air during the course of the experiments.

Results of thermodynamic modeling of the sediment extracts are shown in Table 4.1. Only sediment extract samples in Table 3.11 that had quantifiable concentrations of ${ }^{239} \mathrm{Pu}$ or ${ }^{241} \mathrm{Am}$ were evaluated. In addition, only modeling results that are relevant to the speciation of ${ }^{239} \mathrm{Pu}$ and ${ }^{241} \mathrm{Am}$ are discussed here. Table 4.1 lists the SI values calculated for the most likely phase that could potentially control solubility at equilibrium under the chemical conditions that occurred in the extraction tests and the dominant dissolved species calculated to be in equilibrium with the sediment extracts.

Table 4.1. Results of Thermodynamic Equilibrium Modeling

\begin{tabular}{cccc}
\hline Sample ID & Radionuclide & SI [Phase $]$ & Dominant Dissolved Species \\
\hline B17N54 1 day & ${ }^{241} \mathrm{Am}$ & $-11.8\left[\mathrm{Am}(\mathrm{OH})_{3}(\mathrm{c})\right]$ & $\mathrm{Am}^{3+}(100 \%)$ \\
B17N54 30 day & ${ }^{241} \mathrm{Am}$ & $-12.1\left[\mathrm{Am}(\mathrm{OH})_{3}(\mathrm{c})\right]$ & $\mathrm{Am}^{3+}(100 \%)$ \\
B1HK52 1 day & ${ }^{239} \mathrm{Pu}$ & $-5.5\left[\mathrm{PuO}_{2}(\mathrm{am})\right]$ & $\mathrm{PuO}_{2}{ }^{+}(47 \%), \mathrm{PuO}_{2} \mathrm{NO}_{3}{ }^{0}(25 \%), \mathrm{PuO}_{2}{ }^{2+}(17 \%)$ \\
B1HK52 30 day & ${ }^{241} \mathrm{Am}$ & $-12.2\left[\mathrm{Am}(\mathrm{OH})_{3}(\mathrm{c})\right]$ & $\mathrm{Am}^{3+}(100 \%)$ \\
\hline
\end{tabular}


Results for the three sediment extracts that had measureable ${ }^{241} \mathrm{Am}$ indicate these solutions are highly undersaturated ( $\mathrm{SI}=-11.8$ to -12.2 ) with respect to the $\mathrm{Am}(\mathrm{OH})_{3}(\mathrm{c})$, where $\mathrm{c}$ indicates a crystalline phase, and the free ion $\left(\mathrm{Am}^{3+}\right)$ is the dominant dissolved species. These results indicate that americium solution concentrations are not being controlled by the solubility of $\mathrm{Am}(\mathrm{OH})_{3}(\mathrm{c})$, but instead it is likely that desorption of americium adsorbed to the sediments during the period of active disposal controls the concentration of americium in solutions in contact with these sediments. Sediment extracts that had measureable concentrations of americium occurred only in samples that were fairly acidic ( $\mathrm{pH} 4.34$ to 4.55). Because cations are less effectively adsorbed at lower $\mathrm{pH}$ values, these results suggest that americium will remain effectively sequestered to sediments when $\mathrm{pH}$ conditions approach those of normal Hanford Site groundwater (mildly alkaline, pH 8).

The one sediment extract that had measureable ${ }^{239} \mathrm{Pu}$ was significantly undersaturated with respect to $\mathrm{PuO}_{2}(\mathrm{am})$ [SI = -5.5], although not to the extent that $\mathrm{Am}(\mathrm{OH})_{3}(\mathrm{c})$ was undersaturated (where am indicates x-ray amorphous precipitate, or hydrous oxide $\mathrm{PuO}_{2} \cdot \mathrm{xH}_{2} \mathrm{O}$, or aged precipitates that include both amorphous and microcrystalline $\mathrm{PuO}_{2}$ fractions). Note that $\mathrm{PuO}_{2}(\mathrm{c})$ is not stable because $\alpha$-radiation damage leads to amorphization of crystalline compounds. Dissolved plutonium species were dominated by complexes of the $\mathrm{Pu}(\mathrm{V})$ oxidation state such as $\mathrm{PuO}_{2}{ }^{+}$and $\mathrm{PuO}_{2} \mathrm{NO}_{3}{ }^{0}$, with significant concentrations of $\mathrm{Pu}(\mathrm{VI})$ complexes also occurring ( e.g., $\mathrm{PuO}_{2}{ }^{2+}$ ). Based on the data available at the time this report was published, several explanations could account for this highly unsaturated condition. Similar to americium, plutonium concentrations could be controlled by desorption under acidic conditions. However, this hypothesis does not appear to be consistent with all the data collected in this study. For example, sample B1HK52 30 day had a lower $\mathrm{pH}$ of 4.55, but no measureable plutonium in the extract. Another possible explanation is that dissolved plutonium in the extracts occurs in the form of soluble complexes with TBP degradation products, such as dibutyl phosphate (DBP), or monobutyl phosphate (MBP) that have desorbed from the surfaces of the sediments. This explanation does not appear to be likely because DBP and MBP are expected to be associated with sediments containing high concentrations of TBP. Based on the total organic carbon results in Table 3.3, it is unlikely that sediment sample B1HK52 had a high TBP concentration.

The best explanation for the dissolved concentrations of plutonium observed in the sediment extract is based on recent critical reviews of the solubility of plutonium hydroxides/ hydrous oxides and redox reactions by Neck et al. (2007a, 2007b). These reviews indicate the presently available database for plutonium (the NEA database used in the calculations conducted in this study; Lemire et al. 2001, Guillaumont et al. 2003) is not sufficient to fully explain the solubility behavior of plutonium under all environmentally relevant conditions of $\mathrm{pH}$ and Eh. Among the conclusions by Neck et al. (2007a, 2007b), they find that $\mathrm{Pu}(\mathrm{OH})_{3}(\mathrm{am})$ is not stable except under extremely reducing conditions and converts to $\mathrm{PuO}_{2}(\mathrm{am})$. Under reducing conditions, $\mathrm{PuO}_{2}(\mathrm{am})$ is in equilibrium with both aqueous $\mathrm{Pu}(\mathrm{III})$ and $\mathrm{Pu}(\mathrm{IV})$ species. In the absence of strongly reducing and oxidizing conditions, but in the presence of traces of $\mathrm{O}_{2}(\mathrm{~g})$, total dissolved plutonium concentrations at $\mathrm{pH}>3$ are dominated by $\mathrm{Pu}(\mathrm{V})$ and the solubility is controlled by the mixed valent $\left(\mathrm{Pu}^{\mathrm{V}}\right)_{2 \mathrm{x}}\left(\mathrm{Pu}^{\mathrm{IV}}\right)_{1-2 \mathrm{x}} \mathrm{O}_{2+\mathrm{x}}(\mathrm{am})$ solid. Small neutrally charged $\mathrm{Pu}(\mathrm{IV}) \mathrm{O}_{2}(\mathrm{am})$ colloids/polymers are also present in neutral to alkaline solutions at a constant level of $\log [\mathrm{Pu}(\mathrm{IV})]_{\text {coll }}=-8.3 \pm 1.0$ and play an important role for the redox potentials in these systems. For more conclusions and additional information, see Neck et al. (2007a, 2007b).

Because it is not clear if the $\left(\mathrm{Pu}^{\mathrm{V}}\right)_{2 \mathrm{x}}\left(\mathrm{Pu}^{\mathrm{IV}}\right)_{1-2 \mathrm{x}} \mathrm{O}_{2+\mathrm{x}}(\mathrm{am})$ solid occurs in the sediments examined here, or what the value of $\mathrm{x}$ would be if this solid does exist in these sediments, it is not possible to determine the solubility of this phase in these systems. However, it is interesting to note that $\log [\mathrm{Pu}(\mathrm{IV})]_{\mathrm{coll}}=-8.3 \pm 1.0$ 
is equal to a concentration range of 0.12 to12 $\mu \mathrm{g} / \mathrm{L}$. The concentration of ${ }^{239} \mathrm{Pu}$ measured in the B1HK52 (1 day) groundwater extract $(0.15 \mu \mathrm{g} / \mathrm{L})$ falls within this range. It is well known that these neutrally charged oxyhydroxide polymer/colloids have a high adsorption affinity for oxide and hydroxide mineral surfaces (Neck et al. 2007a, 2007b; Clark et al. 2006). This could explain why the ${ }^{239} \mathrm{Pu}$ concentration measured in the B1HK52 (1 day) groundwater extract is at the low end of the concentration range indicated by $\log [\mathrm{Pu}(\mathrm{IV})]_{\mathrm{coll}}=-8.3 \pm 1.0$ and why the ${ }^{239} \mathrm{Pu}$ concentrations measured other extracts were undetectable. 



\subsection{Summary and Conclusions}

Various analyses were conducted on selected sediment samples collected from two wells (299-W15-46 and 299-W15-48) drilled near the 216-Z-9 Trench to elucidate the form and potential for plutonium and americium to be mobilized under present conditions, and in future remediation scenarios. Analyses included moisture content, determination of the less than sand-size fraction (silt plus clay), carbon analysis, SEM/EDS analysis, microwave-assisted acid digestions for total element analysis, and extraction tests using Hanford Site groundwater as the leachate. Results of the extraction tests were used as input to conduct equilibrium geochemical modeling of the solutions using Geochemist’s Workbench ${ }^{\circledR}$. Geochemical modeling results for plutonium were evaluated in terms of recent conclusions regarding the solubility and redox reactions of plutonium by Neck et al. (2007a, 2007b).

The highest concentrations of plutonium and americium were associated with sediments of low silt/clay content located above silt/clay rich layers within the sediment profile. Researchers also found that plutonium and americium were relatively enriched in the silt/clay portion of these samples (see Table 3.6). SEM/EDS analysis indicated the plutonium and americium in these sediments does not occur as discrete micron-size particles.

Leaching of these sediment samples with Hanford Site groundwater indicates release of plutonium and americium from the sediments correlates most significantly with the acidity of the water and not the initial concentrations of plutonium and americium in the sediments. Only extracts that were acidic after contact with the sediments ( $\mathrm{pH} 4.3$ to 5.4) contained detectable concentrations of extractable plutonium and americium. Water extracts from samples containing high concentrations of TBP indicate that if the TBP degradation products DBP and MBP are available in these sediments, they do not significantly increase the extractability of plutonium or americium.

Geochemical modeling results indicate the americium concentrations in water in contact with these sediments is highly undersaturated with respect to $\mathrm{Am}(\mathrm{OH})_{3}(\mathrm{c})$. It is likely that desorption of americium adsorbed to the sediments during the period of active waste water disposal is what controls americium concentrations in solutions in contact with these sediments. Sediment extracts that had measureable concentrations of americium only occurred in samples that were fairly acidic ( $\mathrm{pH} 4.3$ to 4.6), indicating that americium will remain effectively sequestered to sediments when $\mathrm{pH}$ conditions approach those of normal Hanford Site groundwater (mildly alkaline, pH 8).

The geochemical modeling results indicate that plutonium in extracts in contact with acidic sediment is significantly undersaturated with respect to $\mathrm{PuO}_{2}(\mathrm{am})$. However, recent reviews of plutonium solubility and redox reactions indicate the data used for these calculations is incomplete (Neck et al. 2007a, 2007b). The results of Neck et al. (2007a, 2007b) indicate that plutonium concentrations in solutions in contact with the 216-Z-9 Trench sediment samples might be controlled by a mixed valent solid phase $\left[\left(\mathrm{Pu}^{\mathrm{V}}\right)_{2 \mathrm{x}}\left(\mathrm{Pu}^{\mathrm{IV}}\right)_{1-2 \mathrm{x}} \mathrm{O}_{2+\mathrm{x}}(\mathrm{am})\right]$ with various dissolved $\mathrm{Pu}(\mathrm{V})$ complexes and $\mathrm{Pu}(\mathrm{IV}) \mathrm{O}_{2}(\mathrm{am})$ colloids or nanoclusters being the dominant species in solution for typical Hanford Site groundwater conditions. Adsorption is likely to have a major impact on the potential for these species to remain in solution. Both $\mathrm{Pu}(\mathrm{V})$ complexes and $\mathrm{Pu}(\mathrm{IV}) \mathrm{O}_{2}(\mathrm{am})$ colloids or nanoclusters are well known for their high adsorption affinity for oxide and hydroxide mineral surfaces (Neck et al. 2007a, 2007b; Clark et al. 2006; 
Kaplan et al. 2006; Powell et al. 2005). ${ }^{1,2,3}$ As a result, these species are not likely to remain in solution as $\mathrm{pH}$ values approach those of typical Hanford Site groundwater (mildly alkaline, pH 8).

${ }^{1}$ Clark DL, SS Hecker, GD Jarvinen and MP Neu. 2006. "Plutonium.” Chapter 7 in The Chemistry of the Actinide and Transactinide Elements, $3^{\text {rd }}$ ed., pp. 813-1264, eds LR Morss, NM Edelstein, J Fuger, and JJ Katz, Springer, Netherlands.

${ }^{2}$ Kaplan DI, BA Powell, L Gumapas, JT Coates, RA Fjeld, and DP Diprete. 2006. "Influence of pH on Plutonium Desorption/Solubilization from Sediment.” Environmental Science \& Technology 40:5937-5942.

${ }^{3}$ Powell BA, RA Fjeld, DI Kaplan, JT Coates and SM Serkiz. 2005. "Pu(V)O ${ }_{2}^{+}$Adsorption and Reduction by Synthetic Hematite and Goethite.” Environmental Science \& Technology 39:2107-2114. 


\subsection{Path Forward}

To provide maximum understanding of Pu and Am behavior at the 200-PW-1 OU, the following work is recommended. It is important to determine with certainty if Pu in the 216-Z-9 sediments is in the form of a soluble precipitate or is adsorbed to mineral surfaces. From the results of the work presented here it is not clear if $\mathrm{Pu}$ in the 216-Z-9 sediments occurs as adsorbed species or as a mixed valent solid phase $\left[\left(\mathrm{Pu}^{\mathrm{V}}\right)_{2 \mathrm{x}}\left(\mathrm{Pu}^{\mathrm{IV}}\right)_{1-2 \mathrm{x}} \mathrm{O}_{2+\mathrm{x}}(\mathrm{am})\right]$. If the Pu does occur as a mixed valent solid phase $\left[\left(\mathrm{Pu}^{\mathrm{V}}\right)_{2 \mathrm{x}}\left(\mathrm{Pu}^{\mathrm{IV}}\right)_{1-2 \mathrm{x}} \mathrm{O}_{2+\mathrm{x}}(\mathrm{am})\right]$, the value of $\mathrm{x}$ must be determined to calculate it solubility under various conditions. Synchrotron based $x$-ray adsorption spectroscopy techniques will be applied to determine if $\mathrm{Pu}$ occurs as an adsorbed species or as the mixed valent solid phase $\left[\left(\mathrm{Pu}^{\mathrm{V}}\right)_{2 \mathrm{x}}\left(\mathrm{Pu}^{\mathrm{IV}}\right)_{1-2 \mathrm{x}} \mathrm{O}_{2+\mathrm{x}}(\mathrm{am})\right]$ in the 216-Z-9 sediments. These techniques can also be used to determine the value of $\mathrm{x}$. With this data geochemical modeling can be used predict the solubility of Pu in Hanford vadose zone pore water and groundwater.

Another important issue to resolve is the impact of Pu nanoclusters (Soderholm et al. 2008). Extracts from three sediment samples were examined for the potential for colloidal particles to form and act as agents for transport of $\mathrm{Pu}$. No filterable colloidal size $\mathrm{Pu}(>0.0018 \mu)$ was observed in the three samples examined; however, no dissolved Pu was observed from these three samples either. It is recommended that these experiments be repeated with the samples in which significant concentrations of Pu were observed in the sediment extracts to evaluate if the Pu that occurs in these solutions is monomeric, polymeric nanoclusters, or colloidal. Because Pu nanoclusters are small enough to pass through the $0.0018 \mu$ filters, experiments will be designed to determine if Pu nanoclusters have passed through these filters. Included among the test methods that will be used to confirm the presence of Pu nanoclusters is flow through ion exchange columns. Because Pu nanoclusters are neutrally charged and do not interact with ion-exchange materials, they are expected to pass through these columns.

It is also recommended that adsorption of Pu nanoclusters on 216-Z-9 sediments be studied. It is expected that under typical Hanford groundwater conditions Pu nanoclusters will readily adsorb to mineral surfaces in Hanford sediments. Because no data regarding adsorption of Pu nanoclusters onto the mineral surfaces in Hanford sediments is currently available, it would be very valuable to have such information.

The impact of TBP degradation products on Pu mobility remains a source of uncertainty. Although the findings presented here indicate that mobilization of Pu by TBP degradation products does not appear to be likely, the concentration and water solubility of these compounds remains unknown. Phosphorus-31 NMR techniques should be applied to measure the presence of these compounds in the 216-Z-9 sediment samples. If significant concentrations of these compounds are found, their concentrations in water extracts should be determined and their potential for mobilizing Pu evaluated. 



\subsection{References}

ASTM D2216-98. 1998. Standard Test Method for Laboratory Determination of Water (Moisture) Content of Soil and Rock by Mass. American Society for Testing and Materials, West Conshohocken, Pennsylvania.

ASTM D3987-85. 1999. Standard Test Method for Shake Extraction of Solid Waste with Water. American Society for Testing and Materials, West Conshohocken, Pennsylvania.

ASTM E1915-01. 2001. Standard Test Methods for Analysis of Metal Bearing Ores and Related Materials by Combustion Infrared Absorption Spectrometry. American Society for Testing and Materials, West Conshohocken, Pennsylvania.

Bethke CM and S Yeakel. 2007. The Geochemist’s Workbench ${ }^{\circledR}$, Release 7.0, Reference Manual. Hydrogeology Program, University of Illinois, Urbana, Illinois.

Bowles NA and VJ Rohay. 2007. Borehole Summary Report for Slant Well 299-W15-48 (Borehole C3427) Drilled at the 216-Z-9 Trench. WMP-30566 (Rev. 0), Fluor Hanford, Inc., Richland, Washington.

Caron ME. 2005. Borehole Summary Report for Well 299-W15-46 (C3426) Drilled at the 216-Z-9 Trench. WMP-26264, Rev. 0, Fluor Hanford, Inc., Richland, Washington.

Clark DL, SS Hecker, GD Jarvinen and MP Neu. 2006. "Plutonium.” Chapter 7 in The Chemistry of the Actinide and Transactinide Elements, $3^{\text {rd }}$ ed., pp. 813-1264, eds LR Morss, NM Edelstein, J Fuger, and JJ Katz, Springer, Netherlands.

Clesceri LS, AE Greenberg, and AD Eaton. 1998. Standard Methods for the Examination of Water and Wastewater, 20th Edition. Standard Method 2320 B, “Alkalinity by Titration.” American Public Health Association, American Water Works Association, and Water Environment Federation, Washington, D.C.

Comprehensive Environmental Response, Compensation, and Liability Act of 1980. 1980. Public Law 96-510, as amended, 42 USC 9601 et seq.

DOE/RL. 2007. Remedial Investigation Report for the Plutonium/Organic-Rich Process Condensate/ Process Waste Group Operable Unit: Includes the 200-PW-1, 200-PW-3, and 200-PW-6 Operable Units. DOE/RL-2006-51, Rev. 0, U.S. Department of Energy, Richland Operations Office, Richland, Washington.

Elsner M, SB Haderlein, T Kellerhals, S Luzi, L Zwank, W Angst, and RP Schwarzenbach. 2003. "Mechanisms and Products of Surface-Mediated Reductive Dehalogenation of Carbon Tetrachloride by Fe(II) on Goethite. Environmental Science and Technology 38:2058-2066.

EPA. 1996. "Method 3052, Microwave Assisted Acid Digestion of Siliceous and Organically Based Matrices,” Rev. 0. In Test Methods for Evaluating Solid Waste: Physical/Chemical Methods. EPA SW-846, U.S. Environmental Protection Agency, Office of Solid Waste and Emergency Response, 
Washington, D.C. Accessed September 16, 2008, at

http://www.epa.gov/waste/hazard/testmethods/sw846/pdfs/3052.pdf.

EPA. 2004. "Method 9040C, pH Electrometric Measurement," Rev. 3. In Test Methods for Evaluating Solid Waste: Physical/Chemical Methods. EPA SW-846, U.S. Environmental Protection Agency, Office of Solid Waste and Emergency Response, Washington, D.C. Accessed September 16, 2008, at http://www.epa.gov/waste/hazard/testmethods/sw846/pdfs/9040c.pdf.

EPA. 2007. "Method 9056A, Determination of Inorganic Anions by Ion Chromatography,” Rev. 1. In Test Methods for Evaluating Solid Waste: Physical/Chemical Methods. EPA SW-846, U.S. Environmental Protection Agency, Office of Solid Waste and Emergency Response, Washington, D.C. Accessed September 16, 2008, at http://www.epa.gov/waste/hazard/testmethods/sw846/pdfs/9056a.pdf.

Grenthe I, W Stumm, M Laaksuharju, AC Nilsson, and P Wikberg. 1992. "Redox Potentials and Redox Reactions in Deep Groundwater Systems.” Chemical Geology 98:131.

Guillaumont R, T Fanghänel, J Fuger, I Grenthe, V Neck, DA Palmer, and MH Rand. 2003. “Chemical Thermodynamics," Vol. 5. In Update on the Chemical Thermodynamics of Uranium, Neptunium, Plutonium, Americium and Technetium. Organization for Economic Co-operation and Development/ Nuclear Energy Agency-Thermochemical Database Project, Elsevier, North-Holland, Amsterdam.

Kaplan DI, BA Powell, L Gumapas, JT Coates, RA Fjeld, and DP Diprete. 2006. "Influence of pH on Plutonium Desorption/Solubilization from Sediment.” Environmental Science \& Technology 40:5937-5942.

Lemire RJ, J Fuger, H Nitsche, P Potter, MH Rand, J Rydberg, K Spahiu, JC Sullivan, WJ Ullman, P Vitorge, and H Wanner. 2001. "Chemical Thermodynamics,” Vol. 4. In Chemical Thermodynamics of Neptunium and Plutonium. Organization for Economic Co-operation and Development/Nuclear Energy Agency-Thermochemical Database Project, Elsevier, North-Holland, Amsterdam.

Lindberg RD and DD Runnells. 1984. "Ground Water Redox Reactions: An Analysis of Equilibrium State Applied to Eh Measurements and Geochemical Modeling.” Science 225:925-927

Neck V, M Altmaier, A Seibert, JI Yun, CM Marquardt and T Fanghänel. 2007a. "Solubility and Redox Reactions of $\mathrm{Pu}(\mathrm{IV})$ Hydrous Oxides: Evidence for the Formation of $\mathrm{PuO}_{2+\mathrm{x}}$ (s,hyd)." Radiochimica Acta 95:193-207.

Neck V, M Altmaier, and T Fanghänel. 2007b. "Solubility of Plutonium Hydroxides/Hydrous Oxides Under Reducing Conditions and in the Presence of Oxygen.” Comptes rendus Chimie 10:959-977.

Powell BA, RA Fjeld, DI Kaplan, JT Coates, and SM Serkiz. 2005. " $\mathrm{Pu}(\mathrm{V}) \mathrm{O}_{2}{ }^{+}$Adsorption and Reduction by Synthetic Hematite and Goethite.” Environmental Science \& Technology 39:2107-2114.

Soderholm L, PM Amond, S Kkanthankumar, RE Wilson and PC Burns. "The Structure of the Plutonium Oxide Nanocluster $\left[\mathrm{Pu}_{38} \mathrm{O}_{56} \mathrm{Cl}_{54}\left(\mathrm{H}_{2} \mathrm{O}\right)_{8}\right]^{14-}$.” Angewandte Chemie 47:298-302. 
Appendix A

Geochemical Modeling Output Files 



\section{Appendix A}

\section{Geochemical Modeling Output Files}

All geochemical calculations were performed using the React module of the geochemical modeling code Geochemist’s Workbench $^{\circledR}$ release 7.0.0 (Bethke and Yeaken 2007) using the thermo_NEA.dat thermodynamic database.

\section{Results Output for Groundwater Extract Sample B17N54 1 Day.}

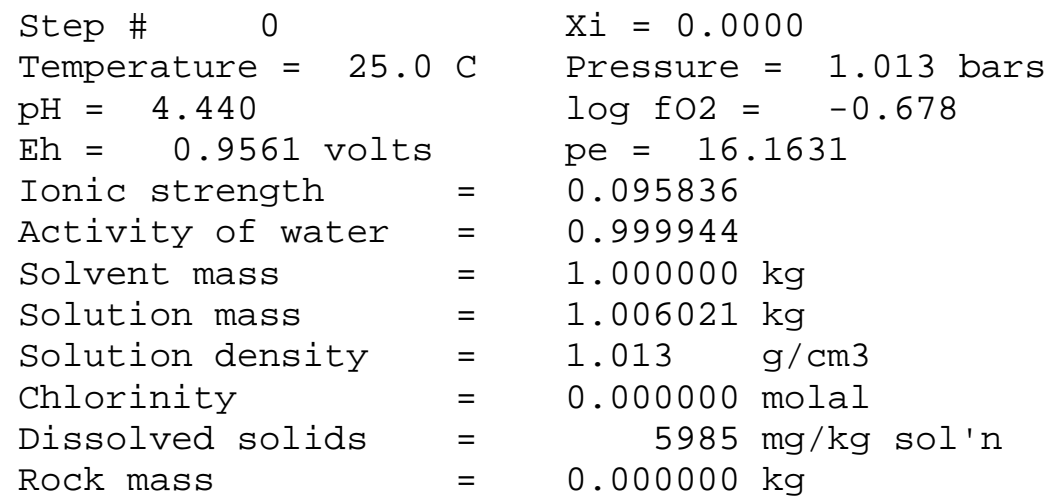

No minerals in system.

\begin{tabular}{|c|c|c|c|c|}
\hline Aqueous species & molality & $\mathrm{mg} / \mathrm{kg} \mathrm{sol'n}$ & act. coef. & $\log$ act. \\
\hline N03 - & 0.07256 & 4472. & 0.7641 & -1.2562 \\
\hline $\mathrm{Na}+$ & $\odot .02821$ & 644.7 & 0.7796 & -1.6577 \\
\hline $\mathrm{Ca}++$ & 0.01554 & 619.1 & 0.4095 & -2.1963 \\
\hline $\mathrm{Mg}++$ & 0.005967 & 144.2 & $\odot .4531$ & -2.5681 \\
\hline $\mathrm{Cl}-$ & 0.001571 & 55.37 & 0.7641 & -2.9207 \\
\hline $\mathrm{K}+$ & $\odot .0005437$ & 21.13 & 0.7641 & -3.3815 \\
\hline $02(a q)$ & ๑. .0००2149 & 6.835 & 1.0000 & -3.6678 \\
\hline $\mathrm{Mn++}$ & $\odot .0 \odot \odot 2099$ & 11.46 & 0.4095 & -4.0658 \\
\hline $\mathrm{Al}+++$ & $\odot .0001807$ & 4.846 & 0.1832 & -4.4801 \\
\hline $\mathrm{H}+$ & $4.349 \mathrm{e}-005$ & 0.04357 & 0.8348 & -4.4400 \\
\hline $\mathrm{AlOH}+2$ & $3.949 \mathrm{e}-005$ & 1.727 & 0.2363 & -5.0301 \\
\hline $\mathrm{Sr}++$ & $1.569 \mathrm{e}-\odot \odot 5$ & 1.366 & 0.3853 & -5.2186 \\
\hline $\mathrm{Ba}++$ & $4.897 e-\odot \odot 6$ & $\odot .6684$ & $\odot .3853$ & -5.7242 \\
\hline SrNO3+1 & $3.012 e-0 \odot 6$ & $\odot .448 \odot$ & $\odot .7019$ & -5.6748 \\
\hline $\mathrm{AlOH} 2+1$ & $2.842 \mathrm{e}-\odot \odot 6$ & $\odot .1723$ & $\odot .7019$ & -5.7001 \\
\hline $\mathrm{Mn}(\mathrm{NO3}) 2$ & 1. $052 \mathrm{e}-0 \odot 6$ & $\odot .1871$ & $1.0 \odot \odot \odot$ & -5.9781 \\
\hline $\mathrm{MnCl}+1$ & $5.947 \mathrm{e}-0 \odot 7$ & 0.05343 & $\odot .7019$ & -6.3794 \\
\hline AlOH3 & $6.916 \mathrm{e}-008$ & ๑. .005362 & 1.0000 & -7.1602 \\
\hline SrCl+1 & $6.522 \mathrm{e}-009$ & $\odot .0007979$ & 0.7019 & -8.3393 \\
\hline $\mathrm{Am}+++$ & $3.399 \mathrm{e}-\odot \odot 9$ & 0.0008210 & 0.0385 & -9.8834 \\
\hline SO4-- & $6.003 e-010$ & $5.732 \mathrm{e}-005$ & 0.3594 & -9.6660 \\
\hline $\mathrm{OH}-1$ & $3.567 e-010$ & $6.030 \mathrm{e}-006$ & 0.7721 & -9.5600 \\
\hline
\end{tabular}




\begin{tabular}{|c|c|c|c|c|}
\hline CaSO4 & $2.797 \mathrm{e}-010$ & $3.785 e-005$ & 1.0000 & -9.5534 \\
\hline AlOH4-1 & $2.713 e-010$ & $2.563 e-0 \odot 5$ & 0.7019 & -9.7202 \\
\hline MgOH+1 & $1.704 \mathrm{e}-010$ & $6.999 \mathrm{e}-006$ & $\odot .7019$ & -9.9221 \\
\hline MnCl2 & $1.361 \mathrm{e}-010$ & $1.703 e-005$ & $1.0 \odot \odot \odot$ & -9.8661 \\
\hline MgSO4 & $1.037 \mathrm{e}-010$ & 1. $241 \mathrm{e}-005$ & $1.000 \odot$ & -9.9841 \\
\hline $\mathrm{MnOH}+1$ & $8.668 e-011$ & $6.199 \mathrm{e}-006$ & $\odot .7019$ & -10.2158 \\
\hline $\mathrm{CaOH}+1$ & $6.300 \mathrm{e}-011$ & $3.575 \mathrm{e}-006$ & 0.7019 & -10.3543 \\
\hline N2 (aq) & $4.541 e-011$ & $1.265 e-006$ & $1.000 \odot$ & -10.3428 \\
\hline NaSO4-1 & $3.388 e-011$ & 4. $010 \mathrm{e}-0 \odot 6$ & 0.7019 & -10.6237 \\
\hline AlS04+1 & $1.066 \mathrm{e}-011$ & 1. 303e- - & $\odot .7019$ & -11.1261 \\
\hline SrCl2 & $8.711 \mathrm{e}-012$ & 1. 373e-๑०6 & $1.0 \odot \odot \odot$ & -11.0599 \\
\hline $\mathrm{AmOH}+2$ & $6.069 e-012$ & $1.569 \mathrm{e}-006$ & ๑. 2363 & -11.8435 \\
\hline MnSO4 & 3. $374 \mathrm{e}-012$ & $5.065 e-007$ & 1.0000 & -11.4718 \\
\hline HSO4-1 & 1. $083 e-012$ & $1.045 e-007$ & 0.7019 & -12.1191 \\
\hline$M n+3$ & 1. $012 \mathrm{e}-012$ & $5.525 e-\odot \odot 8$ & 0.0385 & -13.4097 \\
\hline KS04-1 & $9.039 e-013$ & 1. $214 \mathrm{e}-\odot \odot 7$ & 0.7019 & -12.1976 \\
\hline SrS04 & $4.627 e-013$ & $8.449 e-008$ & 1.0000 & -12.3347 \\
\hline MnCl3-1 & $1.050 \mathrm{e}-013$ & $1.683 e-0 \odot 8$ & $\odot .7019$ & -13.1327 \\
\hline N02-1 & $1.677 \mathrm{e}-014$ & $7.670 \mathrm{e}-010$ & 0.7641 & -13.8923 \\
\hline $\mathrm{SrOH}+1$ & 1. 393e-014 & 1. $449 \mathrm{e}-\odot \odot 9$ & 0.7932 & -13.9567 \\
\hline $\mathrm{BaOH}+1$ & $3.247 e-015$ & $4.981 \mathrm{e}-010$ & $\odot .7019$ & -14.6423 \\
\hline $\mathrm{Am}(\mathrm{OH}) 2+$ & $1.123 e-015$ & $3.091 \mathrm{e}-010$ & 0.7019 & -15.1035 \\
\hline Mn04-1 & $3.613 e-016$ & $4.271 e-011$ & 0.7721 & -15.5545 \\
\hline $\mathrm{HClO}(\mathrm{AQ})$ & $2.140 \mathrm{e}-017$ & $1.116 \mathrm{e}-012$ & 1.0000 & -16.6695 \\
\hline Als042-1 & $1.826 e-019$ & $3.977 e-014$ & $\odot .7019$ & -18.8922 \\
\hline Clo-1 & $3.193 e-020$ & $1.633 e-015$ & 0.7019 & -19.6495 \\
\hline MnO4 - 2 & $1.963 e-022$ & $2.321 e-017$ & 0.2363 & -22.3336 \\
\hline $\mathrm{Sr}(\mathrm{OH}) 2$ & $1.450 \mathrm{e}-025$ & $1.753 \mathrm{e}-020$ & 1.0000 & -24.8387 \\
\hline $\mathrm{Am}(\mathrm{OH}) 3$ & $6.862 \mathrm{e}-026$ & $2.006 \mathrm{e}-020$ & 1.0000 & -25.1635 \\
\hline Cl03-1 & $4.068 e-026$ & $3.374 \mathrm{e}-021$ & 0.7019 & -25.5443 \\
\hline MnOH3-1 & $4.054 e-026$ & $4.270 \mathrm{e}-021$ & 0.7019 & -25.5458 \\
\hline Cl04-1 & $1.847 \mathrm{e}-026$ & $1.826 e-021$ & 0.7019 & -25.8872 \\
\hline Cl02-1 & $5.865 e-029$ & $3.932 \mathrm{e}-024$ & 0.7019 & -28.3854 \\
\hline $\mathrm{HCl02}(\mathrm{AQ}$ & $1.366 e-031$ & $9.298 e-027$ & $1.0 \odot \odot \odot$ & -30.8644 \\
\hline $\mathrm{H} 2(\mathrm{aq})$ & $4.404 \mathrm{e}-045$ & $8.825 e-042$ & $1.00 \odot \odot$ & -44.3561 \\
\hline HSO3-1 & $4.619 e-052$ & $3.722 \mathrm{e}-047$ & 0.7019 & -51.4891 \\
\hline S03-2 & $1.444 \mathrm{e}-054$ & $1.149 \mathrm{e}-049$ & 0.3726 & -54.2691 \\
\hline $\mathrm{H} 2 \mathrm{SO3}(\mathrm{AQ}$ & $8.144 \mathrm{e}-055$ & $6.645 e-050$ & 1.0000 & -54.0891 \\
\hline $\mathrm{NH} 4+1$ & 1. $991 \mathrm{e}-056$ & $3.571 \mathrm{e}-052$ & 0.7555 & -55.8226 \\
\hline NH3 (AQ) & 2. 401e-061 & $4.064 e-057$ & $1.000 \odot$ & -60.6196 \\
\hline NH4SO4-1 & $5.213 e-065$ & $5.912 \mathrm{e}-06 \odot$ & $\odot .7019$ & -64.4366 \\
\hline H2S(AQ) & $2.044 e-143$ & $6.925 e-139$ & 1.0000 & -142.6895 \\
\hline HS-1 & $7.497 e-146$ & $2.465 e-141$ & $\odot .7721$ & -145.2375 \\
\hline S203-2 & $2.648 e-155$ & $2.951 \mathrm{e}-150$ & ๑. 3594 & -155.0215 \\
\hline HS2O3-1 & 1. $915 e-158$ & $2.154 \mathrm{e}-153$ & 0.7019 & -157.8715 \\
\hline$S-2$ & $4.127 e-160$ & 1. $316 e-155$ & 0.3853 & -159.7985 \\
\hline
\end{tabular}

Mineral saturation states

$\log Q / K \quad \log Q / K$

PYROLUSI

BIRNESIT

MANGANIT

GIBBSITE

\begin{abstract}
4.4703s/sat
$2.4233 \mathrm{~s} / \mathrm{sat}$

$0.1503 \mathrm{~s} / \mathrm{sat}$

$0.0698 \mathrm{~s} / \mathrm{sat}$
\end{abstract}

PERICLAS

SRCL2(CR

$\mathrm{SROH} 2$

$\mathrm{CAO}(\mathrm{CR})$
- - - - -

$-15.2681$

$-18.2999$

$-21.3187$

$-26.0163$ 


$\begin{array}{lllr}\text { BARITE } & -5.4103 & \text { LIME_QU } & -26.0163 \\ \text { GYPSUM } & -7.2585 & \text { SRO(CR) } & -38.5687 \\ \text { ANHYDRIT } & -7.4824 & \text { BAO(CR) } & -44.9143 \\ \text { SR(NO3)2 } & -8.1310 & \text { NA(CR) } & -63.7108 \\ \text { CELESTIT } & -8.3047 & \text { K(CR) } & -69.0346 \\ \text { SRSO4 } & -8.4647 & \text { S(CR) } & -106.3243 \\ \text { ALUNITE } & -11.1240 & \text { CA(CR) } & -131.3725 \\ \text { AM }(O H) 3 C & -11.7635 & \text { BA(CR) } & -135.7504 \\ \text { AM }(O H) 3 A & -13.5635 & \text { SR }(C R) & -136.3348 \\ \text { BACL2 }(C R & -13.8656 & \text { ALABANDI } & -144.4462\end{array}$

\begin{tabular}{|c|c|c|}
\hline Gases & fugacity & $\log$ fug. \\
\hline $02(g)$ & 0.2100 & -0.678 \\
\hline $\mathrm{H} 2 \mathrm{O}(\mathrm{G})$ & $\odot .03162$ & -1.500 \\
\hline $\mathrm{N} 2(\mathrm{G})$ & 1. $\odot 89 \mathrm{e}-\odot \odot 7$ & -6.963 \\
\hline $\mathrm{HCL}(\mathrm{G})$ & $2.235 e-014$ & -13.651 \\
\hline $\mathrm{CL} 2(\mathrm{G})$ & $3.198 \mathrm{e}-020$ & -19.495 \\
\hline$C L(G)$ & $6.345 e-029$ & -28.198 \\
\hline $0(\mathrm{G})$ & $1.138 \mathrm{e}-041$ & -40.944 \\
\hline H2 (g) & $6.221 e-042$ & -41.206 \\
\hline S02(G) & $3.697 e-055$ & -54.432 \\
\hline$H(G)$ & $6.123 e-057$ & -56.213 \\
\hline NH3(G) & $3.796 e-063$ & -62.421 \\
\hline $\mathrm{NA}(\mathrm{G})$ & $6.299 e-078$ & -77.201 \\
\hline $\mathrm{K}(\mathrm{G})$ & $2.319 \mathrm{e}-080$ & -79.635 \\
\hline$N(G)$ & $5.112 \mathrm{e}-084$ & -83.291 \\
\hline $\mathrm{H} 2 \mathrm{~S}(\mathrm{G})$ & $2.136 e-142$ & -141.670 \\
\hline$S(G)$ & 1. $606 \mathrm{e}-148$ & -147.794 \\
\hline $\mathrm{CA}(\mathrm{G})$ & $2.498 e-157$ & -156.602 \\
\hline S2(G) & $2.406 e-227$ & -226.619 \\
\hline
\end{tabular}

\begin{tabular}{lrrrr} 
& & \multicolumn{2}{c}{ In fluid } & \multicolumn{2}{c}{ Sorbed } & $\mathrm{Kd}$ \\
Original basis total moles & moles & $\mathrm{mg} / \mathrm{kg}$ & $\mathrm{moles}$ & $\mathrm{mg} / \mathrm{kg}$ \\
$\mathrm{L} / \mathrm{kg}$
\end{tabular}




\begin{tabular}{|c|c|c|c|c|}
\hline \multicolumn{2}{|c|}{ Elemental composition } & \multicolumn{2}{|c|}{ In fluid } & Sorbed \\
\hline & total moles & moles & $\mathrm{mg} / \mathrm{kg}$ & moles $\quad \mathrm{mg} / \mathrm{kg}$ \\
\hline Al & $\odot .0002231$ & $\odot .0 \odot \odot 2231$ & 5.983 & \\
\hline $\mathrm{Am}$ & $3.405 \mathrm{e}-\odot \odot 9$ & $3.405 \mathrm{e}-\odot \odot 9$ & $\odot .0 \odot \odot 8225$ & \\
\hline $\mathrm{Ba}$ & $4.897 e-006$ & $4.897 e-\odot \odot 6$ & 0.6684 & \\
\hline $\mathrm{Ca}$ & ๑.01554 & ๑. 01554 & 619.1 & \\
\hline $\mathrm{Cl}$ & 0.001572 & 0.001572 & 55.39 & \\
\hline Hydrogen & 111.0 & 111.0 & $1.112 \mathrm{e}+005$ & \\
\hline $\mathrm{K}$ & $\odot .0005437$ & $\odot .00 \odot 5437$ & 21.13 & \\
\hline $\mathrm{Mg}$ & 0.005967 & $\odot .0 \odot 5967$ & 144.2 & \\
\hline $\mathrm{Mn}$ & $\odot .0002115$ & $\odot .00 \odot 2115$ & 11.55 & \\
\hline $\mathrm{N}$ & $\odot .07257$ & 0.07257 & 1010. & \\
\hline $\mathrm{Na}$ & 0.02821 & $\odot .02821$ & 644.7 & \\
\hline oxygen & 55.73 & 55.73 & $8.863 e+0 \odot 5$ & \\
\hline $\mathrm{S}$ & 1. $034 \mathrm{e}-\odot \odot 9$ & 1. $\odot 34 \mathrm{e}-\odot \odot 9$ & $3.296 e-\odot \odot 5$ & \\
\hline $\mathrm{Sr}$ & $1.870 \mathrm{e}-\odot \odot 5$ & $1.870 \mathrm{e}-\odot \odot 5$ & 1.629 & \\
\hline
\end{tabular}

\section{Results Output for Groundwater Extract Sample B17N54 30 days.}

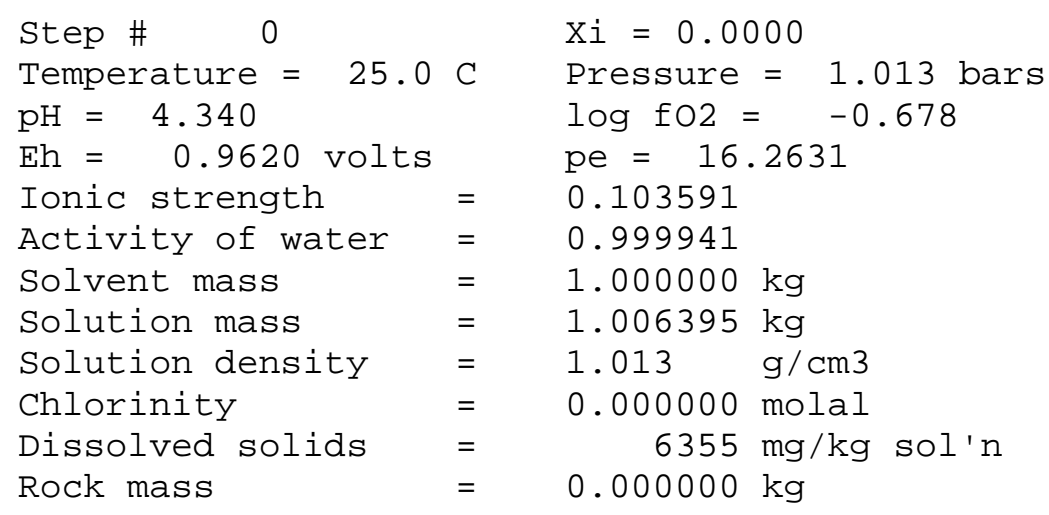

No minerals in system.

\begin{tabular}{|c|c|c|c|c|}
\hline Aqueous species & molality & $\mathrm{mg} / \mathrm{kg}$ sol'n & ct. coef. & $\log$ act. \\
\hline N03 - & $\odot .07644$ & 4709. & $\odot .7582$ & -1.2369 \\
\hline $\mathrm{Na}+$ & $\odot .03064$ & 700.0 & 0.7745 & -1.6246 \\
\hline $\mathrm{Ca}++$ & 0.01688 & 672.4 & $\odot .40 \odot 9$ & -2.1695 \\
\hline $\mathrm{Mg}++$ & $\odot .00650 \odot$ & 157.0 & $\odot .4457$ & -2.5381 \\
\hline $\mathrm{Cl}-$ & $\odot .001675$ & 59.02 & 0.7582 & -2.8961 \\
\hline $\mathrm{K}+$ & $\odot .0 \odot \odot 5972$ & 23.20 & $\odot .7582$ & -3.3440 \\
\hline $\mathrm{Al+++}$ & ๑. . $00 \odot 3318$ & 8.896 & 0.1771 & -4.2309 \\
\hline Mn++ & $0.00 \odot 2314$ & 12.63 & 0.4009 & -4.0326 \\
\hline $02(\mathrm{aq})$ & 0.0002149 & 6.833 & 1.0000 & -3.6678 \\
\hline $\mathrm{AlOH}+2$ & $5.894 e-005$ & 2.576 & 0.2232 & -4.8809 \\
\hline $\mathrm{H}+$ & $5.492 e-005$ & $\odot .05501$ & 0.8322 & -4.3400 \\
\hline Sr++ & $1.646 \mathrm{e}-0 \odot 5$ & 1.433 & 0.3761 & -5.2083 \\
\hline $\mathrm{Ba}++$ & $3.582 \mathrm{e}-\odot \odot 6$ & 0.4887 & 0.3761 & -5.870 \\
\hline SrNO3+1 & $3.269 e-\odot \odot 6$ & 0.4860 & 0.6924 & -5.645 \\
\hline
\end{tabular}




\begin{tabular}{|c|c|c|c|c|}
\hline $\mathrm{AlOH} 2+1$ & $3.226 e-006$ & ๑.1955 & 0.6924 & -5.6509 \\
\hline $\mathrm{Mn}(\mathrm{NO} 3) 2$ & $1.241 \mathrm{e}-0 \odot 6$ & 0.2206 & $1.000 \odot$ & -5.9064 \\
\hline MnCl+1 & $6.886 \mathrm{e}-\odot \odot 7$ & ๑. 06185 & 0.6924 & -6.3217 \\
\hline AlOH3 & $6.153 e-0 \odot 8$ & 0.004769 & $1.00 \odot \odot$ & -7.2109 \\
\hline SrCl+1 & $7.165 \mathrm{e}-009$ & ๑. ๑๑९८762 & 0.6924 & -8.3044 \\
\hline Am+++ & $3.247 e-009$ & ๑. . $0 \odot ७ 7839$ & 0.0338 & -9.9593 \\
\hline SO4 - - & $5.865 e-010$ & $5.598 \mathrm{e}-005$ & 0.3495 & -9.6883 \\
\hline $\mathrm{OH}-1$ & $2.853 e-010$ & $4.822 e-006$ & 0.7667 & -9.6600 \\
\hline CaSO4 & $2.827 e-010$ & $3.824 \mathrm{e}-005$ & 1.0000 & -9.5487 \\
\hline AlOH4-1 & $1.944 \mathrm{e}-010$ & $1.835 \mathrm{e}-005$ & 0.6924 & -9.8710 \\
\hline MnCl2 & $1.645 \mathrm{e}-010$ & $2.057 e-005$ & $1.0 \odot \odot \odot$ & -9.7838 \\
\hline $\mathrm{MgOH}+1$ & $1.471 \mathrm{e}-010$ & $6.038 \mathrm{e}-006$ & $\odot .6924$ & -9.9921 \\
\hline MgSO4 & $1.056 \mathrm{e}-010$ & $1.263 \mathrm{e}-005$ & 1.0000 & -9.9763 \\
\hline N2 (aq) & $7.865 e-011$ & $2.189 \mathrm{e}-006$ & 1.0000 & -10.1043 \\
\hline $\mathrm{MnOH}+1$ & $7.534 \mathrm{e}-011$ & $5.386 e-\odot \odot 6$ & 0.6924 & -10.2826 \\
\hline $\mathrm{CaOH}+1$ & $5.397 e-011$ & 3. $061 \mathrm{e}-006$ & 0.6924 & -10.4275 \\
\hline NaSO4-1 & $3.522 \mathrm{e}-011$ & $4.166 e-006$ & 0.6924 & -10.6129 \\
\hline Als04+1 & $1.822 \mathrm{e}-011$ & $2.227 \mathrm{e}-\odot \odot 6$ & ๑. 6924 & -10.8991 \\
\hline SrCl2 & $9.988 e-012$ & $1.573 e-0 \odot 6$ & $1.0 \odot \odot \odot$ & $-11.00 \odot 5$ \\
\hline $\mathrm{AmOH}+2$ & $4.285 e-012$ & $1.107 \mathrm{e}-006$ & ๑. 2232 & -12.0193 \\
\hline MnS04 & $3.460 \mathrm{e}-012$ & $5.192 \mathrm{e}-0 \odot 7$ & $1.000 \odot$ & -11.4609 \\
\hline$M n+3$ & $1.564 \mathrm{e}-012$ & $8.537 e-008$ & 0.0338 & -13.2765 \\
\hline HSO4-1 & $1.313 e-012$ & $1.266 \mathrm{e}-007$ & 0.6924 & -12.0414 \\
\hline KSO4-1 & $9.491 \mathrm{e}-013$ & $1.275 \mathrm{e}-007$ & 0.6924 & -12.1823 \\
\hline SrSO4 & $4.502 e-013$ & $8.216 e-008$ & 1.0000 & -12.3466 \\
\hline MnCl3-1 & $1.361 e-013$ & $2.181 e-008$ & 0.6924 & -13.0259 \\
\hline NO2-1 & $1.767 \mathrm{e}-014$ & $8.077 \mathrm{e}-010$ & $\odot .7582$ & -13.8730 \\
\hline $\mathrm{SrOH}+1$ & $1.139 \mathrm{e}-014$ & $1.184 \mathrm{e}-009$ & 0.7889 & -14.0464 \\
\hline $\mathrm{BaOH}+1$ & $1.866 \mathrm{e}-015$ & $2.862 \mathrm{e}-010$ & 0.6924 & -14.8886 \\
\hline $\mathrm{Am}(\mathrm{OH}) 2+$ & $6.029 e-016$ & $1.660 \mathrm{e}-010$ & 0.6924 & -15.3794 \\
\hline MnO4-1 & 1. $968 \mathrm{e}-016$ & $2.326 e-011$ & 0.7667 & -15.8214 \\
\hline $\mathrm{HClO}(\mathrm{AQ})$ & $2.851 e-017$ & $1.486 e-012$ & 1.0000 & -16.5450 \\
\hline Als042-1 & $2.966 e-019$ & $6.458 e-014$ & ๑. 6924 & -18.6874 \\
\hline Cl0-1 & $3.425 \mathrm{e}-02 \odot$ & $1.751 \mathrm{e}-015$ & $\odot .6924$ & -19.6250 \\
\hline MnO4-2 & $8.930 \mathrm{e}-023$ & $1.055 e-017$ & 0.2232 & -22.7004 \\
\hline $\mathrm{Sr}(\mathrm{OH}) 2$ & $9.367 e-026$ & $1.132 \mathrm{e}-020$ & $1.000 \odot$ & -25.0284 \\
\hline Cl03-1 & $4.364 \mathrm{e}-026$ & $3.619 e-021$ & 0.6924 & -25.5198 \\
\hline $\mathrm{Am}(\mathrm{OH}) 3$ & $2.888 e-026$ & $8.437 e-021$ & 1.0000 & -25.5394 \\
\hline MnOH3-1 & $2.223 e-026$ & $2.341 e-021$ & 0.6924 & -25.8127 \\
\hline Cl04-1 & 1. $981 \mathrm{e}-026$ & $1.958 e-021$ & 0.6924 & -25.8626 \\
\hline Cl02-1 & $6.292 e-029$ & $4.217 e-024$ & 0.6924 & -28.3609 \\
\hline $\mathrm{HCl02}(\mathrm{AQ}$ & $1.820 \mathrm{e}-031$ & $1.238 \mathrm{e}-026$ & $1.000 \odot$ & -30.7399 \\
\hline H2(aq) & $4.404 \mathrm{e}-045$ & $8.822 \mathrm{e}-042$ & $1.0 \odot \odot \odot$ & -44.3561 \\
\hline HSO3-1 & $5.601 \mathrm{e}-052$ & $4.512 \mathrm{e}-047$ & 0.6924 & -51.4114 \\
\hline S03-2 & 1. $408 \mathrm{e}-054$ & $1.120 \mathrm{e}-049$ & 0.3630 & -54.2914 \\
\hline $\mathrm{H} 2 \mathrm{SO} 3(\mathrm{AQ}$ & $1.226 e-054$ & 1. $00 \odot \mathrm{e}-049$ & $1.000 \odot$ & -53.9114 \\
\hline $\mathrm{NH} 4+1$ & $3.327 e-056$ & $5.963 e-052$ & 0.7492 & -55.6034 \\
\hline NH3 (AQ) & $3.160 \mathrm{e}-061$ & $5.347 e-057$ & $1.0 \odot \odot \odot$ & $-60.50 \odot 4$ \\
\hline NH4SO4-1 & $8.318 e-065$ & $9.431 \mathrm{e}-06 \odot$ & ๑. 6924 & -64.2396 \\
\hline H2S(AQ) & $3.078 e-143$ & $1.042 e-138$ & $1.0 \odot \odot \odot$ & -142.5117 \\
\hline HS-1 & $9.030 \mathrm{e}-146$ & $2.968 e-141$ & $\odot .7667$ & -145.1597 \\
\hline S203-2 & $3.896 e-155$ & $4.341 e-150$ & ๑.3495 & -154.8659 \\
\hline HS2O3-1 & $3.497 e-158$ & $3.931 e-153$ & 0.6924 & -157.6159 \\
\hline S-2 & 4. $018 e-160$ & 1. $280 \mathrm{e}-155$ & ○. 3761 & -159.8207 \\
\hline
\end{tabular}




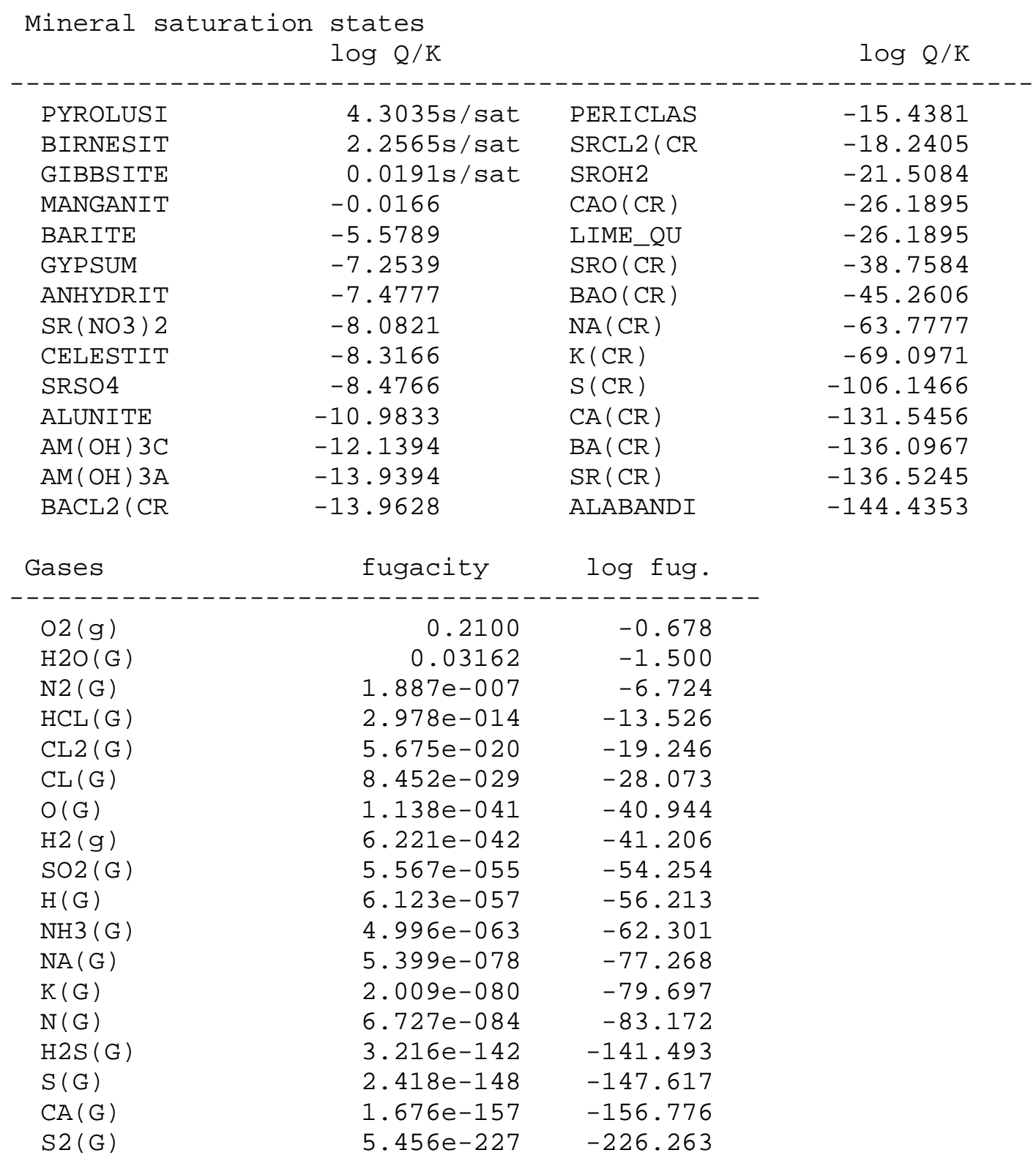

\begin{tabular}{|c|c|c|c|c|c|}
\hline & & In $f$ & Luid & Sorbed & $\mathrm{Kd}$ \\
\hline Original basis & total moles & moles & $\mathrm{mg} / \mathrm{kg}$ & moles $\quad \mathrm{mg} / \mathrm{kg}$ & $\mathrm{L} / \mathrm{kg}$ \\
\hline & & - - - - - - - & & & \\
\hline $\mathrm{Al}+++$ & 0.000394 & $\odot .000394$ & 10.6 & & \\
\hline $\mathrm{Am}+++$ & $3.25 e-\odot \odot 9$ & $3.25 e-0 \odot 9$ & $\odot .000785$ & & \\
\hline $\mathrm{Ba}++$ & $3.58 \mathrm{e}-006$ & $3.58 \mathrm{e}-006$ & 0.489 & & \\
\hline $\mathrm{Ca}++$ & 0.0169 & ๑. 0169 & 672. & & \\
\hline $\mathrm{Cl}-$ & $\odot .00168$ & 0.00168 & 59.0 & & \\
\hline $\mathrm{H}+$ & $-1.07 e-005$ & $-1.07 e-005$ & -0.0107 & & \\
\hline $\mathrm{H} 2 \mathrm{O}$ & 55.5 & 55.5 & $9.94 \mathrm{e}+005$ & & \\
\hline $\mathrm{K}+$ & ๑. 000597 & $\odot .000597$ & 23.2 & & \\
\hline $\mathrm{Mg}++$ & 0.00650 & 0.00650 & 157. & & \\
\hline $\mathrm{Mn++}$ & 0.000233 & 0.000233 & 12.7 & & \\
\hline N03- & $\odot .0764$ & $\odot .0764$ & $4.71 e+0 \odot 3$ & & \\
\hline
\end{tabular}




$\begin{array}{lrrr}\mathrm{Na}+ & 0.0306 & 0.0306 & 700 . \\ \mathrm{O} 2(\mathrm{aq}) & 0.00 \odot 215 & 0.000215 & 6.83 \\ \mathrm{~S} 04-- & 1.03 \mathrm{e}-009 & 1.03 \mathrm{e}-009 & 9.87 \mathrm{e}-005 \\ \mathrm{Sr}++ & 1.97 \mathrm{e}-005 & 1.97 \mathrm{e}-005 & 1.72\end{array}$

Elemental composition In fluid

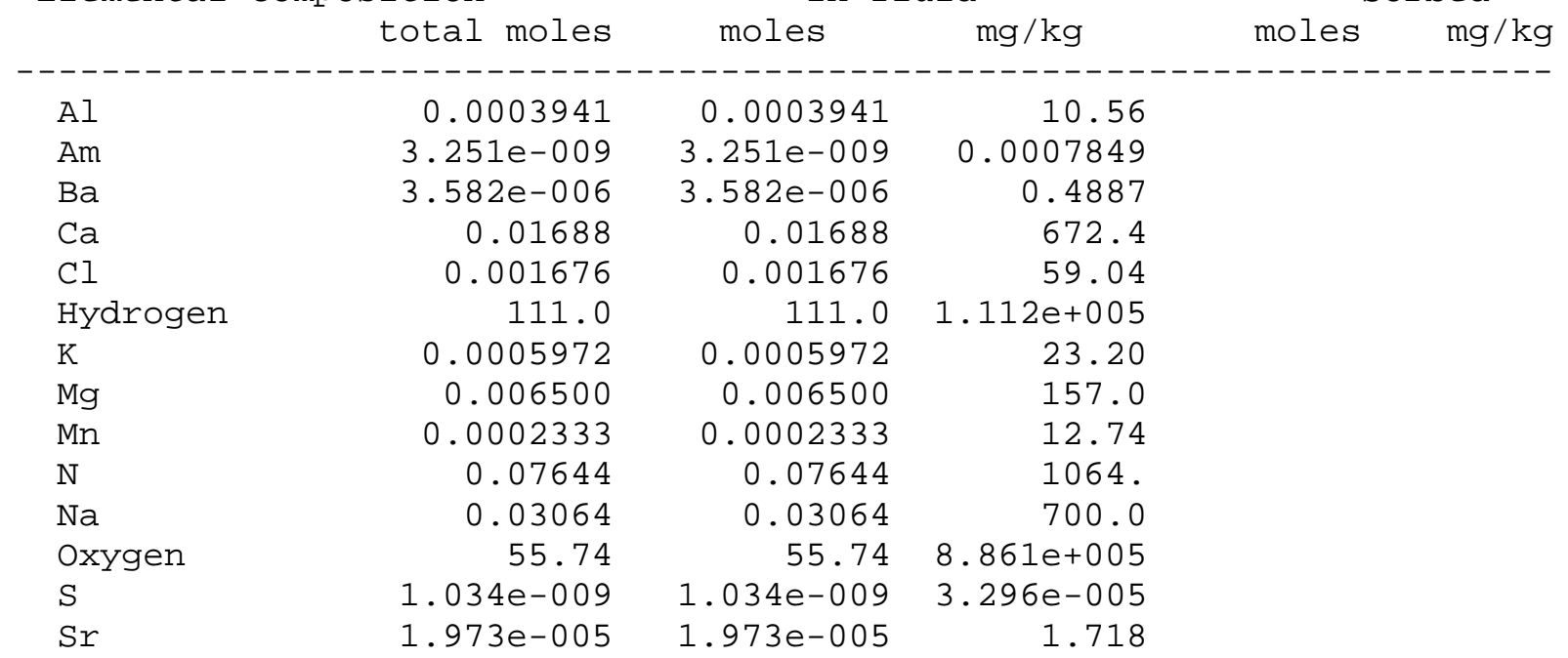

\section{Results Output for Groundwater Extract Sample B1HK52 1 day.}

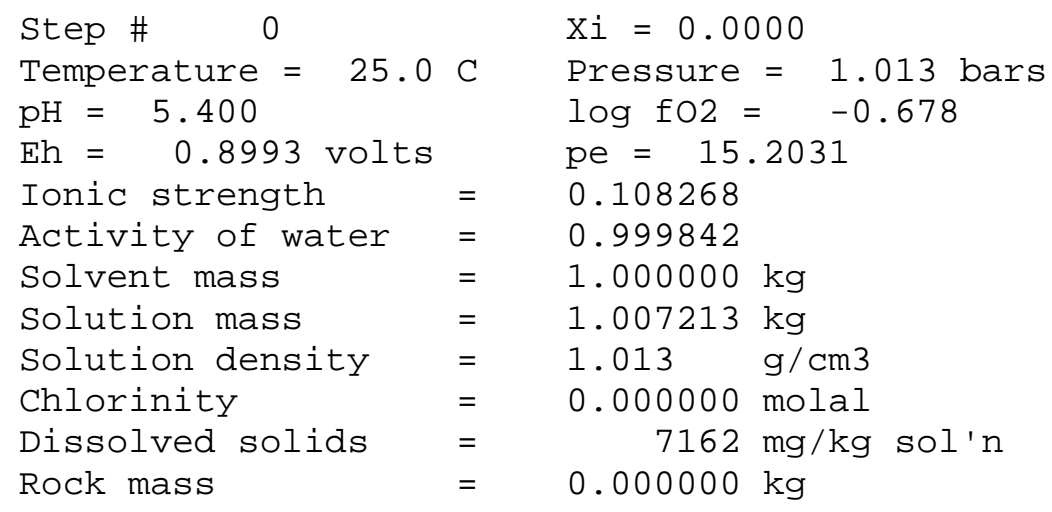

No minerals in system.

$\begin{array}{lrccr}\text { Aqueous species } & \text { molality } & \mathrm{mg} / \mathrm{kg} \text { sol' } \mathrm{n} & \text { act. coef. } & \text { Log act. } \\ \mathrm{NO3-} & 0.08099 & 4986 & 0.7549 & -1.2137 \\ \mathrm{Na}+ & 0.04922 & 1124 . & 0.7717 & -1.4204 \\ \mathrm{Ca}++ & 0.01176 & 467.8 & 0.3961 & -2.3320 \\ \mathrm{Mg}++ & 0.007144 & 172.4 & 0.4415 & -2.5011 \\ \mathrm{Cl}- & 0.004487 & 158.0 & 0.7549 & -2.4701 \\ \text { K+ } & 0.001992 & 77.31 & 0.7549 & -2.8229 \\ \text { S04-- } & 0.0009313 & 88.83 & 0.3439 & -3.4944 \\ \text { CaS04 } & 0.0003038 & 41.07 & 1.00 \odot 0 & -3.5174 \\ \text { 02(aq) } & 0.0002149 & 6.827 & 1.00 \odot \odot & -3.6678\end{array}$




\begin{tabular}{|c|c|c|c|c|}
\hline MgSO4 & $\odot .0001797$ & 21.47 & 1.0000 & -3.7455 \\
\hline NaS04-1 & $8.878 \mathrm{e}-\odot \odot 5$ & 10.49 & $\odot .6869$ & -4.2148 \\
\hline $\mathrm{Mn}++$ & $8.380 e-005$ & 4.571 & 0.3961 & -4.4790 \\
\hline $\mathrm{Sr}++$ & $1.795 \mathrm{e}-005$ & 1.562 & 0.3709 & -5.1765 \\
\hline KS04-1 & $4.964 e-006$ & 0.6661 & 0.6869 & -5.4673 \\
\hline $\mathrm{H}+$ & $4.792 \mathrm{e}-\odot \odot 6$ & 0.004795 & $\odot .8308$ & -5.4000 \\
\hline SrN03+1 & $3.740 \mathrm{e}-\odot \odot 6$ & $\odot .5556$ & 0.6869 & -5.5902 \\
\hline MnSO4 & 1. $935 \mathrm{e}-\odot \odot 6$ & $\odot .2900$ & 1.0000 & -5.7134 \\
\hline $\mathrm{Ba}++$ & $1.789 \mathrm{e}-\odot \odot 6$ & $\odot .2439$ & ๑.3709 & -6.1782 \\
\hline SrSO4 & $7.569 e-007$ & 0.1380 & 1.0000 & -6.1210 \\
\hline MnCl+1 & $6.623 e-0 \odot 7$ & ๑. 05943 & 0.6869 & -6.3421 \\
\hline $\mathrm{Mn}(\mathrm{NO3}) 2$ & $4.940 \mathrm{e}-007$ & 0.08776 & 1.0000 & -6.3063 \\
\hline HSO4-1 & 1. 801e-००7 & $\odot .01736$ & 0.6869 & -6.9075 \\
\hline SrCl+1 & $2 . \odot 72 \mathrm{e}-\odot \odot 8$ & $\odot .0 \odot 2532$ & $\odot .6869$ & -7.8466 \\
\hline $\mathrm{OH}-1$ & $3.289 \mathrm{e}-\odot \odot 9$ & $5.554 \mathrm{e}-\odot \odot 5$ & $\odot .7636$ & -8.6001 \\
\hline $\mathrm{MgOH}+1$ & $1.853 \mathrm{e}-\odot \odot 9$ & $7.601 \mathrm{e}-\odot \odot 5$ & $\odot .6869$ & -8.8952 \\
\hline $\mathrm{CaOH}+1$ & $4.296 \mathrm{e}-010$ & $2.435 \mathrm{e}-\odot \odot 5$ & $\odot .6869$ & -9.5300 \\
\hline MnCl2 & $4.186 e-010$ & $5.230 \mathrm{e}-005$ & 1.0000 & -9.3782 \\
\hline $\mathrm{MnOH}+1$ & $3.119 \mathrm{e}-010$ & $2.228 \mathrm{e}-\odot \odot 5$ & $\odot .6869$ & -9.6690 \\
\hline Pu02+ & $2.901 \mathrm{e}-010$ & $7.804 \mathrm{e}-\odot \odot 5$ & $\odot .6869$ & -9.7006 \\
\hline Pu02N03( & $1.534 \mathrm{e}-010$ & $5.070 \mathrm{e}-0 \odot 5$ & 1.0000 & -9.8143 \\
\hline Pu02+2 & 1. $019 e-010$ & $2.742 \mathrm{e}-0 \odot 5$ & 0.2159 & -10.6575 \\
\hline SrCl2 & $7.643 e-011$ & $1.203 e-005$ & 1.0000 & -10.1168 \\
\hline Pu020H+ & $4.320 \mathrm{e}-011$ & 1. $235 \mathrm{e}-\odot \odot 5$ & 0.6869 & -10.5276 \\
\hline Pu02S04A & $9.955 e-012$ & 3. $628 \mathrm{e}-006$ & 1.0000 & -11.0020 \\
\hline Pu02S04 - & $9.291 e-012$ & $3.386 e-006$ & 0.6869 & -11.1951 \\
\hline Pu02N03+ & 3. $908 e-012$ & 1. $292 \mathrm{e}-006$ & 0.6869 & -11.5712 \\
\hline Pu02Cl(A & $3.383 e-012$ & 1. $029 \mathrm{e}-0 \odot 6$ & 1.0000 & -11.4707 \\
\hline MnCl3-1 & $9.307 e-013$ & 1. $490 \mathrm{e}-007$ & $\odot .6869$ & -12.1943 \\
\hline N2 (aq) & $6.640 \mathrm{e}-013$ & $1.847 \mathrm{e}-008$ & 1.0000 & -12.1778 \\
\hline PuO2OH2 & $4.812 \mathrm{e}-013$ & $1.457 \mathrm{e}-\odot \odot 7$ & $1.000 \odot$ & -12.3177 \\
\hline Pu02S042 & 1. $443 e-013$ & $6.636 \mathrm{e}-\odot \odot 8$ & $\odot .2159$ & -13.5064 \\
\hline SrOH+1 & $1.412 \mathrm{e}-013$ & $1.466 \mathrm{e}-\odot \odot 8$ & 0.7864 & -12.9546 \\
\hline Pu02Cl+ & $1.366 \mathrm{e}-013$ & $4.156 e-\odot \odot 8$ & $\odot .6869$ & -13.0276 \\
\hline Mn04-1 & $1.070 \mathrm{e}-013$ & $1.263 e-\odot \odot 8$ & 0.7636 & -13.0878 \\
\hline$M n+3$ & $5.255 e-014$ & $2.867 e-\odot \odot 9$ & 0.0314 & -14.7829 \\
\hline N02-1 & $1.872 \mathrm{e}-014$ & $8.551 e-010$ & $\odot .7549$ & -13.8498 \\
\hline $\mathrm{BaOH}+1$ & 1. $064 \mathrm{e}-014$ & $1.630 \mathrm{e}-009$ & 0.6869 & -14.1363 \\
\hline $\mathrm{Pu}(\mathrm{OH}) 4$ & $3.517 e-016$ & $1.072 \mathrm{e}-010$ & 1.0000 & -15.4539 \\
\hline Pu020H & $2.508 e-016$ & $7.171 \mathrm{e}-011$ & 1.0000 & -15.6007 \\
\hline $\mathrm{HClO}(\mathrm{AQ})$ & $6.622 \mathrm{e}-018$ & $3.449 e-013$ & 1.0000 & -17.1790 \\
\hline MnO4 - 2 & $5.739 e-019$ & $6.777 e-014$ & 0.2159 & -18.9069 \\
\hline Clo-1 & $9.207 e-020$ & $4.703 e-015$ & 0.6869 & -19.1990 \\
\hline Pu02OH3 - & $8.818 e-02 \odot$ & $2.819 e-014$ & $\odot .6869$ & -19.2177 \\
\hline $\mathrm{PuOH}+3$ & $3.161 \mathrm{e}-022$ & $8.035 e-017$ & 0.0314 & -23.0036 \\
\hline PuS04+2 & $1.852 \mathrm{e}-022$ & $6.162 \mathrm{e}-017$ & ๑. 2159 & -22.3980 \\
\hline $\mathrm{PuO2OH} 2-$ & $4.096 e-023$ & $1.240 \mathrm{e}-017$ & 0.6869 & -22.5508 \\
\hline $\mathrm{Sr}(\mathrm{OH}) 2$ & $1.328 e-023$ & $1.604 \mathrm{e}-018$ & 1.0000 & -22.8767 \\
\hline MnOH3-1 & $1.213 e-023$ & $1.276 \mathrm{e}-018$ & 0.6869 & -23.0792 \\
\hline PuS042AQ & $6.420 \mathrm{e}-024$ & $2.748 e-018$ & $1.000 \odot$ & -23.1924 \\
\hline $\mathrm{Pu}+3$ & $2.437 \mathrm{e}-025$ & $5.782 \mathrm{e}-020$ & 0.0314 & -26.1167 \\
\hline Cl03-1 & $1.173 e-025$ & $9.719 \mathrm{e}-021$ & $\odot .6869$ & -25.0938 \\
\hline Pu++++ & $5.926 e-026$ & $1.406 \mathrm{e}-020$ & $\odot .0 \odot 21$ & -27.9036 \\
\hline Cl04-1 & $5.326 e-026$ & $5.259 e-021$ & 0.6869 & -25.4367 \\
\hline
\end{tabular}




\begin{tabular}{|c|c|c|c|c|}
\hline $\mathrm{PuOH}+2$ & $3.540 \mathrm{e}-027$ & $8.998 e-022$ & 0.2159 & -27.1167 \\
\hline Cl02-1 & $1.691 e-028$ & $1.133 e-023$ & 0.6869 & -27.9349 \\
\hline PuOH2+ & $5.576 e-030$ & $1.511 \mathrm{e}-024$ & 0.6869 & -29.4168 \\
\hline $\mathrm{HClO2}(\mathrm{AQ}$ & $4.228 e-032$ & $2.874 \mathrm{e}-027$ & 1.0000 & -31.3739 \\
\hline $\mathrm{Pu}(\mathrm{OH}) 3$ & $3.042 e-039$ & $8.759 e-034$ & 1.0000 & -38.5169 \\
\hline $\mathrm{H} 2(\mathrm{aq})$ & $4.404 \mathrm{e}-045$ & $8.813 e-042$ & 1.0000 & -44.3562 \\
\hline HSO3-1 & $7.684 \mathrm{e}-047$ & $6.185 e-042$ & 0.6869 & -46.2775 \\
\hline S03-2 & $2.234 \mathrm{e}-048$ & $1.775 \mathrm{e}-043$ & 0.3577 & -48.0975 \\
\hline $\mathrm{H} 2 \mathrm{SO} 3(\mathrm{AQ}$ & $1.454 \mathrm{e}-050$ & $1.185 e-045$ & 1.0000 & -49.8375 \\
\hline $\mathrm{NH} 4+1$ & $2.675 e-058$ & $4.790 e-054$ & 0.7456 & -57.7002 \\
\hline $\mathrm{NH} 4 \mathrm{SO} 04-1$ & $1.048 e-060$ & $1.188 e-055$ & 0.6869 & -60.1426 \\
\hline NH3 (AQ) & $2.903 e-062$ & $4.908 e-058$ & 1.0000 & -61.5372 \\
\hline $\mathrm{H} 2 \mathrm{~S}(\mathrm{AQ})$ & $3.649 e-139$ & 1. $235 e-134$ & 1.0000 & -138.4379 \\
\hline $\mathrm{HS}-1$ & $1.234 \mathrm{e}-140$ & $4.052 e-136$ & 0.7636 & -140.0259 \\
\hline S203-2 & $7.333 e-145$ & $8.164 \mathrm{e}-140$ & 0.3439 & -144.5982 \\
\hline HS2O3-1 & $5.687 e-149$ & $6.388 e-144$ & 0.6869 & -148.4082 \\
\hline S-2 & $6.366 e-154$ & $2.027 e-149$ & 0.3709 & -153.6269 \\
\hline
\end{tabular}

Mineral saturation states

$\log Q / K \quad \log Q / K$

$\begin{array}{llll}\text { PYROLUSI } & 5.9771 \mathrm{~s} / \mathrm{sat} & \text { SROH2 } & -19.3567 \\ \text { BIRNESIT } & 3.9301 \mathrm{~s} / \mathrm{sat} & \text { LIME_QU } & -24.2320 \\ \text { MANGANIT } & 1.6570 \mathrm{~s} / \mathrm{sat} & \text { CAO(CR) } & -24.2320 \\ \text { BARITE } & 0.3074 \mathrm{~s} / \mathrm{sat} & \mathrm{Pu}(\mathrm{OH}) 3 \mathrm{C} & -25.1169 \\ \text { GYPSUM } & -1.2226 & \mathrm{Pu}(\mathrm{OH}) 3 \mathrm{~A} & -26.9169 \\ \text { ANHYDRIT } & -1.4464 & \mathrm{SRO}(\mathrm{CR}) & -36.6066 \\ \text { CELESTIT } & -2.0910 & \mathrm{BAO}(\mathrm{CR}) & -43.4483 \\ \text { SRSO4 } & -2.2510 & \mathrm{NA}(\mathrm{CR}) & -62.5135 \\ \text { PU02(AM) } & -5.4537 & \mathrm{~K}(\mathrm{CR}) & -67.5160 \\ \text { SR(NO3)2 } & -8.0039 & \mathrm{~S}(\mathrm{CR}) & -102.0727 \\ \text { PU02OH } & -9.5107 & \mathrm{CA}(\mathrm{CR}) & -129.5881 \\ \text { PERICLAS } & -13.2812 & \mathrm{BA}(\mathrm{CR}) & -134.2844 \\ \text { BACL2 }(\mathrm{CR} & -13.4184 & \mathrm{SR}(\mathrm{CR}) & -134.3727 \\ \text { SRCL2 }(\mathrm{CR} & -17.3568 & \text { ALABANDI } & -138.6878\end{array}$

\begin{tabular}{lcc} 
Gases & fugacity & log fug. \\
\hline O2(g) & 0.2100 & -0.678 \\
H2O(G) & 0.03162 & -1.500 \\
N2(G) & $1.593 e-009$ & -8.798 \\
HCL(G) & $6.917 \mathrm{e}-015$ & -14.160 \\
CL2(G) & $3.062 \mathrm{e}-021$ & -20.514 \\
CL(G) & $1.963 \mathrm{e}-029$ & -28.707 \\
O(G) & $1.138 \mathrm{e}-041$ & -40.944 \\
H2(g) & $6.220 \mathrm{e}-042$ & -41.206 \\
SO2(G) & $6.600 \mathrm{e}-051$ & -50.180 \\
H(G) & $6.122 \mathrm{e}-057$ & -56.213 \\
NH3(G) & $4.590 \mathrm{e}-064$ & -63.338 \\
NA(G) & $9.921 \mathrm{e}-077$ & -76.003 \\
K(G) & $7.657 \mathrm{e}-079$ & -78.116 \\
N(G) & $6.181 \mathrm{e}-085$ & -84.209 \\
H2S(G) & $3.812 \mathrm{e}-138$ & -137.419 \\
S(G) & $2.866 \mathrm{e}-144$ & -143.543
\end{tabular}




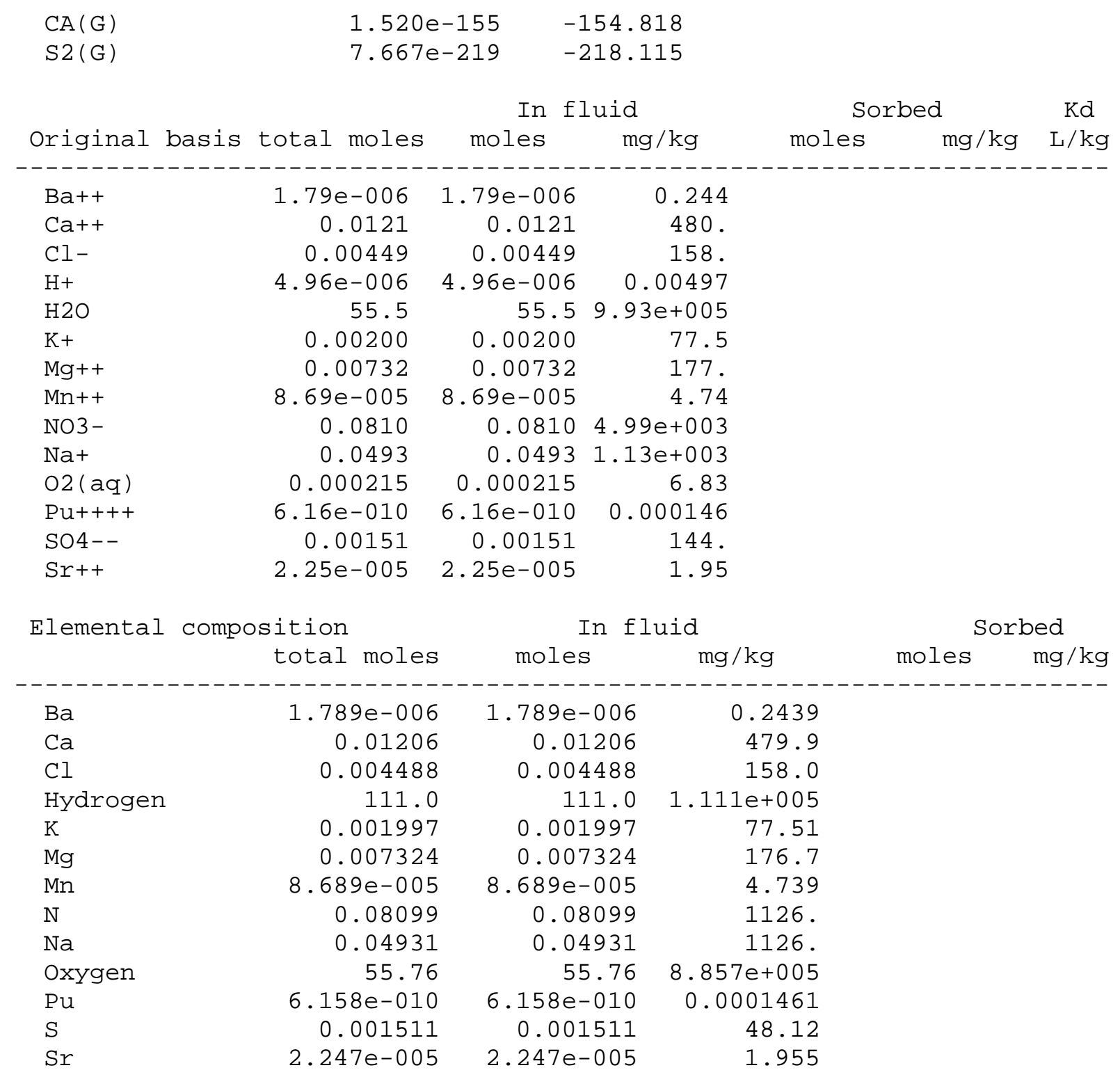

Results Output for Groundwater Extract Sample B1HK52 30 days.

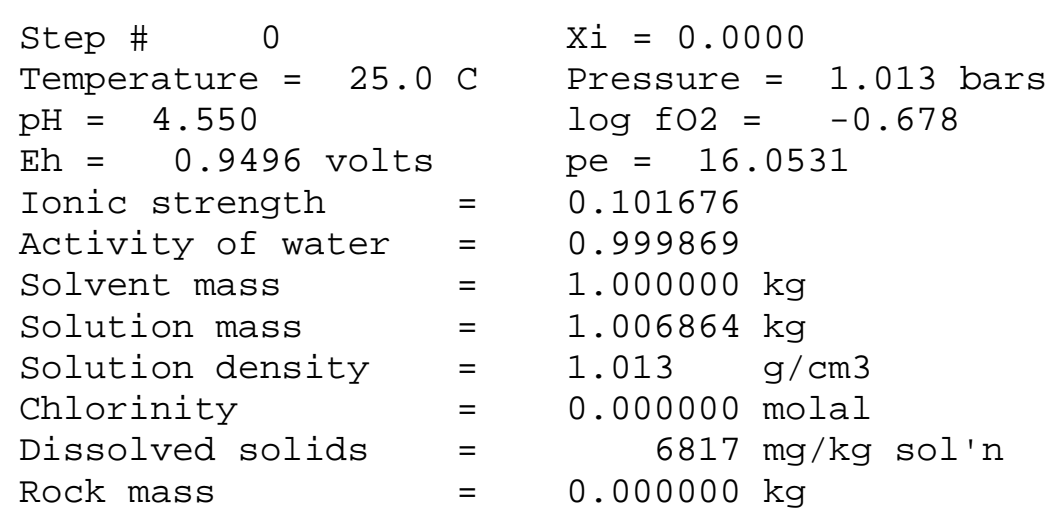


No minerals in system.

\begin{tabular}{|c|c|c|c|c|}
\hline Aqueous $s p$ & molality & $\mathrm{mg} / \mathrm{kg}$ sol'n & act. coef. & $\log$ act. \\
\hline & & & & \\
\hline N03 - & $\odot .08465$ & 5213. & 0.7596 & -1.1918 \\
\hline $\mathrm{Na}+$ & $\odot .03066$ & 700.0 & 0.7758 & -1.6237 \\
\hline $\mathrm{Ca}++$ & 0.01114 & 443.3 & 0.4030 & -2.3480 \\
\hline $\mathrm{Mg}++$ & ๑. 008998 & 217.2 & 0.4474 & -2.3951 \\
\hline $\mathrm{Cl}-$ & $\odot .00370 \odot$ & 130.3 & 0.7596 & -2.5512 \\
\hline $\mathrm{K}+$ & $\odot .0 \odot 2299$ & 89.26 & 0.7596 & -2.7579 \\
\hline $\mathrm{Mn}++$ & ๑ . $0 \odot \odot 23 \odot 4$ & 12.57 & 0.4030 & -4.0323 \\
\hline $02(\mathrm{aq})$ & 0.0002149 & 6.829 & 1.0000 & -3.6678 \\
\hline $\mathrm{Al}+++$ & $4.574 \mathrm{e}-0 \odot 5$ & 1.226 & 0.1785 & -5.0879 \\
\hline $\mathrm{H}+$ & $3.384 e-005$ & 0.03388 & $\odot .8329$ & -4.5500 \\
\hline $\mathrm{Sr++}$ & $1.616 \mathrm{e}-\odot \odot 5$ & 1.406 & $\odot .3783$ & -5.2138 \\
\hline $\mathrm{AlOH}+2$ & 1. $310 \mathrm{e}-\odot \odot 5$ & $\odot .5724$ & $\odot .2263$ & -5.5280 \\
\hline SrNO3+1 & $3.570 \mathrm{e}-\odot \odot 6$ & $\odot .5305$ & $\odot .6947$ & -5.6056 \\
\hline $\mathrm{Ba}++$ & $3.439 \mathrm{e}-\odot \odot 6$ & $\odot .469 \odot$ & $\odot .3783$ & -5.8858 \\
\hline $\mathrm{Mn}(\mathrm{N} 03) 2$ & $1.528 \mathrm{e}-\odot \odot 6$ & $\odot .2716$ & $1.00 \odot \odot$ & -5.8159 \\
\hline $\mathrm{MnCl}+1$ & $1.519 \mathrm{e}-\odot \odot 6$ & $\odot .1364$ & $\odot .6947$ & -5.9765 \\
\hline $\mathrm{AlOH} 2+1$ & $1.175 \mathrm{e}-006$ & 0.07120 & 0.6947 & -6.0880 \\
\hline AlOH3 & $3.647 e-008$ & 0.002825 & 1.0000 & -7.4381 \\
\hline $\mathrm{SrCl}+1$ & $1.560 \mathrm{e}-008$ & $\odot .001907$ & 0.6947 & -7.9650 \\
\hline $\mathrm{MnCl2}$ & $8.059 e-010$ & ๑. . $00 \odot 1007$ & 1.0000 & -9.0937 \\
\hline $\mathrm{Am}+++$ & $6.613 e-010$ & ๑. . 0001596 & 0.0349 & -10.6367 \\
\hline SO4 - - & $6.262 \mathrm{e}-010$ & $5.974 \mathrm{e}-005$ & $\odot .3519$ & -9.6569 \\
\hline $\mathrm{OH}-1$ & $4.620 \mathrm{e}-010$ & $7.803 e-0 \odot 6$ & 0.7680 & -9.4501 \\
\hline $\mathrm{MgOH}+1$ & 3. $304 \mathrm{e}-010$ & $1.356 e-005$ & 0.6947 & -9.6392 \\
\hline CaS04 & $2.014 \mathrm{e}-010$ & $2.724 \mathrm{e}-\odot \odot 5$ & $1.0 \odot \odot \odot$ & -9.6959 \\
\hline $\mathrm{AlOH} 4-1$ & $1.862 \mathrm{e}-010$ & $1.757 \mathrm{e}-0 \odot 5$ & 0.6947 & -9.8881 \\
\hline MgSO4 & $1.577 \mathrm{e}-010$ & $1.886 e-\odot \odot 5$ & $1.0 \odot \odot \odot$ & -9.8020 \\
\hline $\mathrm{MnOH}+1$ & $1.219 \mathrm{e}-010$ & $8.707 \mathrm{e}-\odot \odot 6$ & $\odot .6947$ & -10.0724 \\
\hline $\mathrm{CaOH}+1$ & $5.783 e-011$ & $3.279 \mathrm{e}-\odot \odot 6$ & $\odot .6947$ & -10.3960 \\
\hline $\mathrm{SrCl} 2$ & $4.828 e-011$ & $7.6 \odot 2 \mathrm{e}-\odot \odot 6$ & 1.0000 & -10.3162 \\
\hline $\mathrm{NaSO} 4-1$ & $3.781 \mathrm{e}-011$ & $4.470 \mathrm{e}-\odot \odot 6$ & $\odot .6947$ & -10.5806 \\
\hline N2 ( aq) & $3.681 \mathrm{e}-011$ & $1.024 \mathrm{e}-0 \odot 6$ & 1.0000 & -10.4340 \\
\hline KSO4-1 & $3.921 \mathrm{e}-012$ & $5.263 e-0 \odot 7$ & 0.6947 & -11.5649 \\
\hline MnS04 & $3.722 \mathrm{e}-012$ & $5.582 \mathrm{e}-\odot \odot 7$ & 1.0000 & -11.4292 \\
\hline AlS04+1 & $2.713 e-012$ & $3.315 e-007$ & 0.6947 & -11.7248 \\
\hline MnCl3-1 & $1.470 \mathrm{e}-012$ & $2.355 e-\odot \odot 7$ & 0.6947 & -11.9909 \\
\hline $\mathrm{AmOH}+2$ & 1. $441 \mathrm{e}-012$ & $3.720 \mathrm{e}-\odot \odot 7$ & 0.2263 & -12.4868 \\
\hline$M n+3$ & $9.352 e-013$ & $5.103 e-0 \odot 8$ & 0.0349 & -13.4862 \\
\hline HSO4-1 & $8.673 e-013$ & $8.362 \mathrm{e}-0 \odot 8$ & 0.6947 & -12.2200 \\
\hline SrS04 & $4.778 e-013$ & $8.717 e-\odot \odot 8$ & $1.000 \odot$ & -12.3207 \\
\hline N02-1 & $1.957 e-014$ & $8.940 \mathrm{e}-010$ & $\odot .7596$ & -13.8279 \\
\hline $\mathrm{SrOH}+1$ & $1.822 \mathrm{e}-014$ & $1.893 e-009$ & $\odot .7899$ & -13.8419 \\
\hline $\mathrm{BaOH}+1$ & $2.913 e-015$ & $4.465 e-01 \odot$ & $\odot .6947$ & -14.6938 \\
\hline MnO4-1 & $8.386 e-016$ & $9.906 \mathrm{e}-011$ & 0.7680 & -15.1911 \\
\hline $\mathrm{Am}(\mathrm{OH}) 2+$ & $3.322 e-016$ & $9.139 e-011$ & $\odot .6947$ & -15.6368 \\
\hline $\operatorname{HClO}(A Q)$ & $3.890 \mathrm{e}-017$ & $2.027 e-012$ & 1.0000 & -16.4101 \\
\hline Clo-i & $7.553 e-020$ & $3.859 e-015$ & 0.6947 & -19.2801 \\
\hline Als042-1 & $4.747 e-020$ & $1.033 e-014$ & 0.6947 & -19.4817 \\
\hline MnO4-2 & $6.097 e-022$ & $7.202 \mathrm{e}-017$ & 0.2263 & -21.8602 \\
\hline $\mathrm{Sr}(\mathrm{OH}) 2$ & $2.433 e-025$ & $2.939 e-020$ & 1.0000 & -24.6139 \\
\hline
\end{tabular}




\begin{tabular}{|c|c|c|c|c|}
\hline Cl03-1 & $9.623 e-026$ & $7.976 e-021$ & 0.6947 & -25.1749 \\
\hline MnOH3-1 & $9.457 \mathrm{e}-026$ & $9.952 \mathrm{e}-021$ & 0.6947 & -25.1825 \\
\hline Cl04-1 & $4.369 e-026$ & $4.316 e-021$ & 0.6947 & -25.5178 \\
\hline $\mathrm{Am}(\mathrm{OH}) 3$ & $2.589 \mathrm{e}-026$ & $7.560 \mathrm{e}-021$ & $1.000 \odot$ & -25.5869 \\
\hline ClO2-1 & $1.387 e-028$ & $9.295 e-024$ & 0.6947 & -28.0160 \\
\hline $\mathrm{HClO2}(\mathrm{AQ}$ & $2.483 e-031$ & $1.688 \mathrm{e}-026$ & 1.0000 & -30.6050 \\
\hline $\mathrm{H} 2(\mathrm{aq})$ & $4.404 \mathrm{e}-045$ & $8.817 e-042$ & 1.0000 & -44.3562 \\
\hline HSO3-1 & $3.700 \mathrm{e}-\odot 52$ & $2.979 \mathrm{e}-047$ & $\odot .6947$ & -51.5900 \\
\hline S03-2 & $1.504 \mathrm{e}-054$ & $1.196 e-049$ & 0.3653 & -54.2600 \\
\hline $\mathrm{H} 2 \mathrm{SO} 3(\mathrm{AQ}$ & $5.012 \mathrm{e}-055$ & $4.085 e-05 \odot$ & 1.0000 & -54.3000 \\
\hline $\mathrm{NH} 4+1$ & $1.400 \mathrm{e}-056$ & $2.509 e-052$ & 0.7507 & -55.9783 \\
\hline NH3 (AQ) & $2.161 \mathrm{e}-061$ & $3.656 \mathrm{e}-057$ & $1.000 \odot$ & -60.6653 \\
\hline $\mathrm{NH} 4 \mathrm{SO}^{-1}-1$ & $3.758 \mathrm{e}-065$ & $4.259 \mathrm{e}-06 \odot$ & $\odot .6947$ & -64.5832 \\
\hline $\mathrm{H} 2 \mathrm{~S}(\mathrm{AQ})$ & $1.258 \mathrm{e}-143$ & $4.258 e-139$ & 1.0000 & -142.9003 \\
\hline HS-1 & $5.975 e-146$ & 1. $963 e-141$ & 0.7680 & -145.3383 \\
\hline S203-2 & $1.70 \odot \mathrm{e}-155$ & $1.893 e-150$ & $\odot .3519$ & -155.2232 \\
\hline HS2O3-1 & $9.441 e-159$ & 1. $061 e-153$ & $\odot .6947$ & -158.1832 \\
\hline S-2 & 4. $293 e-16 \odot$ & $1.367 e-155$ & 0.3783 & -159.7893 \\
\hline
\end{tabular}

Mineral saturation states

$\log \mathrm{Q} / \mathrm{K}$

$\log \mathrm{Q} / \mathrm{K}$

$\begin{array}{llll}\text { PYROLUSI } & 4.7238 \mathrm{~s} / \mathrm{sat} & \text { PERICLAS } & -14.8752 \\ \text { BIRNESIT } & 2.6768 \mathrm{~s} / \mathrm{sat} & \text { SRCL2(CR } & -17.5562 \\ \text { MANGANIT } & 0.4037 \mathrm{~s} / \mathrm{sat} & \text { SROH2 } & -21.0939 \\ \text { GIBBSITE } & -0.2081 & \text { CAO(CR) } & -25.9480 \\ \text { BARITE } & -5.5627 & \text { LIME_QU } & -25.9480 \\ \text { GYPSUM } & -7.4011 & \text { SRO(CR) } & -38.3439 \\ \text { ANHYDRIT } & -7.6249 & \text { BAO(CR) } & -44.8558 \\ \text { SR(NO3)2 } & -7.9974 & \text { NA(CR) } & -63.5668 \\ \text { CELESTIT } & -8.2907 & \text { K(CR) } & -68.3010 \\ \text { SRSO4 } & -8.4507 & \text { S(CR) } & -106.5352 \\ \text { ALUNITE } & -11.6459 & \text { CA(CR) } & -131.3041 \\ \text { AM }(\text { OH }) 3 C & -12.1869 & \text { BA(CR) } & -135.6919 \\ \text { BACL2 }(C R & -13.2882 & \text { SR(CR) } & -136.1100 \\ \text { AM }(\text { OH }) 3 A & -13.9869 & \text { ALABANDI } & -144.4036\end{array}$

\begin{tabular}{lrr} 
Gases & fugacity & log fug \\
\hline O2(g) & 0.2100 & -0.678 \\
H2O(G) & 0.03162 & -1.500 \\
N2(G) & $8.830 \mathrm{e}-008$ & -7.054 \\
HCL(G) & $4.062 \mathrm{e}-014$ & -13.391 \\
CL2(G) & $1.056 \mathrm{e}-019$ & -18.976 \\
CL(G) & $1.153 \mathrm{e}-028$ & -27.938 \\
O(G) & $1.138 \mathrm{e}-041$ & -40.944 \\
H2(g) & $6.221 \mathrm{e}-042$ & -41.206 \\
SO2(G) & $2.275 \mathrm{e}-055$ & -54.643 \\
H(G) & $6.122 \mathrm{e}-057$ & -56.213 \\
NH3(G) & $3.418 \mathrm{e}-063$ & -62.466 \\
NA(G) & $8.774 \mathrm{e}-078$ & -77.057 \\
K(G) & $1.256 \mathrm{e}-079$ & -78.901 \\
N(G) & $4.602 \mathrm{e}-084$ & -83.337 \\
H2S(G) & $1.314 \mathrm{e}-142$ & -141.881
\end{tabular}




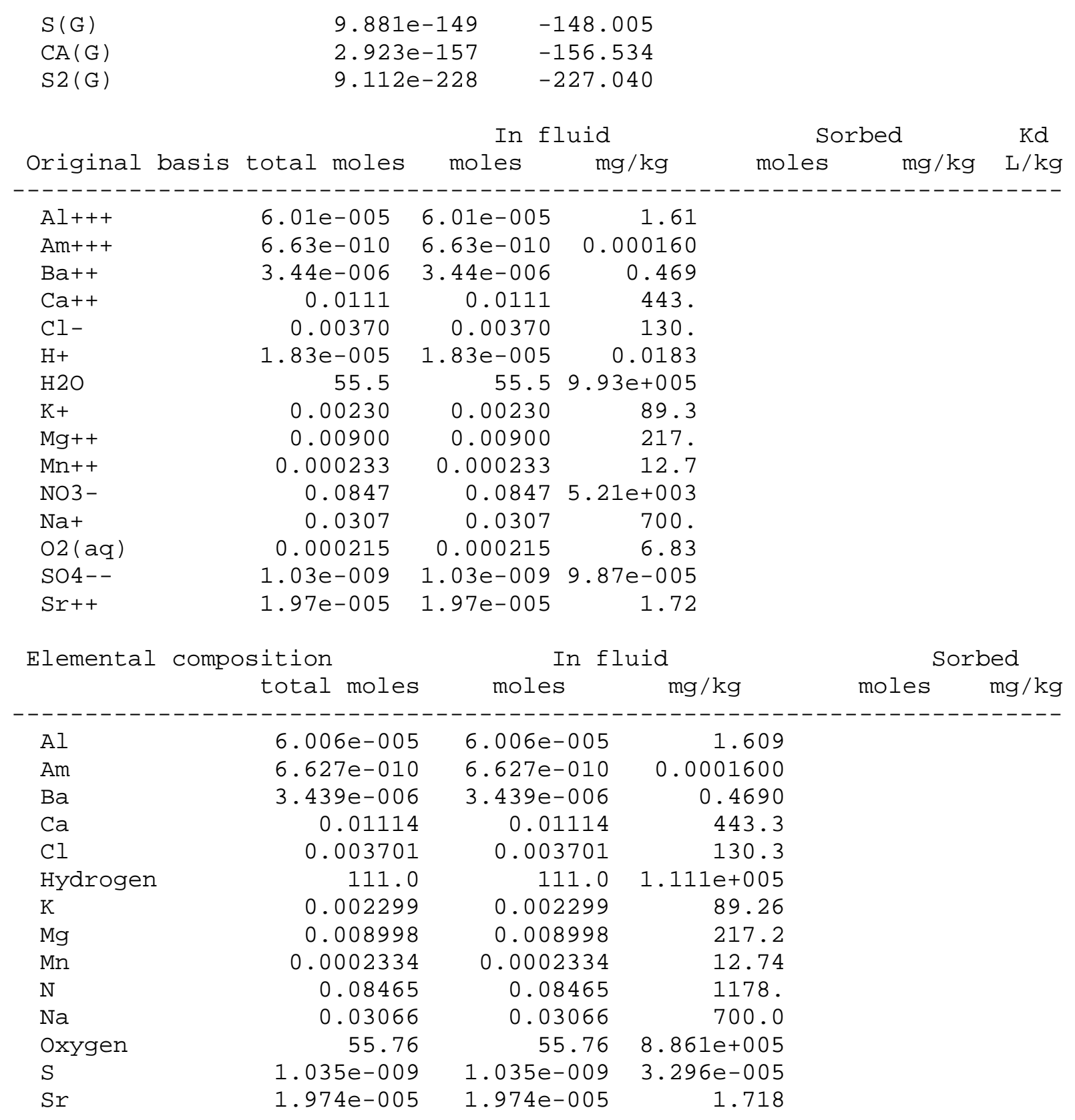




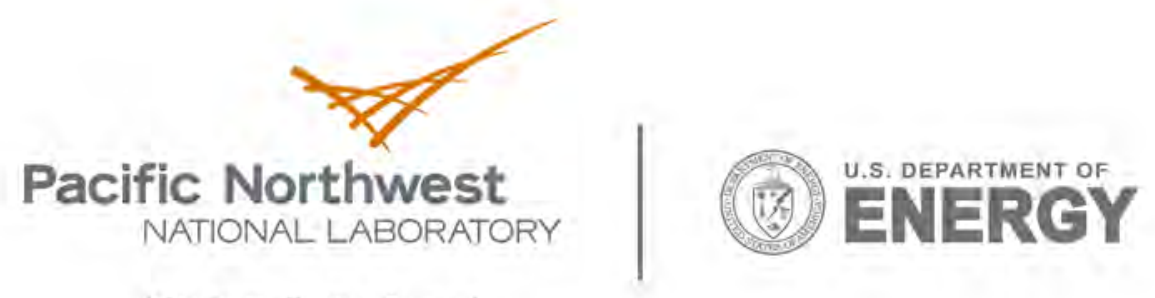

902 Battelle Boulevard

P.O. Box 999

Richland, WA 99352

1-888-375-PNNL (7665)

www.pnl.gov 